\title{
Whitham's Method and Dubrovin-Novikov Bracket in Single-Phase and Multiphase Cases ${ }^{\star}$
}

\author{
Andrei Ya. MALTSEV
}

L.D. Landau Institute for Theoretical Physics, $1 A$ Ak. Semenova Ave., Chernogolovka, Moscow reg., 142432, Russia

E-mail: maltsev@itp.ac.ru

Received April 23, 2012, in final form December 11, 2012; Published online December 24, 2012 http://dx.doi.org/10.3842/SIGMA.2012.103

\begin{abstract}
In this paper we examine in detail the procedure of averaging of the local fieldtheoretic Poisson brackets proposed by B.A. Dubrovin and S.P. Novikov for the method of Whitham. The main attention is paid to the questions of justification and the conditions of applicability of the Dubrovin-Novikov procedure. Separate consideration is given to special features of single-phase and multiphase cases. In particular, one of the main results is the insensitivity of the procedure of bracket averaging to the appearance of "resonances" which can arise in the multi-phase situation.
\end{abstract}

Key words: quasiperiodic solutions; slow modulations; Hamiltonian structures

2010 Mathematics Subject Classification: 37K05; 35B10; 35B15; 35B34; 35L65

\section{Introduction}

As is well-known, the Whitham method [48-50] is associated with slow modulations of periodic or quasiperiodic $m$-phase solutions of nonlinear systems

$$
F^{i}\left(\boldsymbol{\varphi}, \boldsymbol{\varphi}_{t}, \boldsymbol{\varphi}_{x}, \ldots\right)=0, \quad i=1, \ldots, n, \quad \boldsymbol{\varphi}=\left(\varphi^{1}, \ldots, \varphi^{n}\right),
$$

which are usually represented in the form

$$
\varphi^{i}(x, t)=\Phi^{i}\left(\mathbf{k}(\mathbf{U}) x+\boldsymbol{\omega}(\mathbf{U}) t+\boldsymbol{\theta}_{0}, \mathbf{U}\right) .
$$

Let us note that we will consider here systems with one spatial variable $x$ and one time variable $t$. In these notations the functions $\mathbf{k}(\mathbf{U})$ and $\boldsymbol{\omega}(\mathbf{U})$ play the role of the "wave numbers" and "frequencies" of $m$-phase solutions, while the parameters $\boldsymbol{\theta}_{0}$ represent the "initial phase shifts". The parameters $\mathbf{U}=\left(U^{1}, \ldots, U^{N}\right)$ can be chosen in an arbitrary way, we just assume that they do not change under shifts of the initial phases of solutions $\boldsymbol{\theta}_{0}$.

The functions $\Phi^{i}(\boldsymbol{\theta})$ satisfy the system

$$
F^{i}\left(\boldsymbol{\Phi}, \omega^{\alpha} \boldsymbol{\Phi}_{\theta^{\alpha}}, k^{\beta} \boldsymbol{\Phi}_{\theta^{\beta}}, \ldots\right) \equiv 0, \quad i=1, \ldots, n,
$$

and we have to choose for each value of $\mathbf{U}$ some function $\boldsymbol{\Phi}(\boldsymbol{\theta}, \mathbf{U})$ as having "zero initial phase shift". The corresponding set of $m$-phase solutions of (1.1) can be then represented in the form (1.2). For $m$-phase solutions of (1.1) we have in this case $\mathbf{k}(\mathbf{U})=\left(k^{1}(\mathbf{U}), \ldots, k^{m}(\mathbf{U})\right)$, $\boldsymbol{\omega}(\mathbf{U})=\left(\omega^{1}(\mathbf{U}), \ldots, \omega^{m}(\mathbf{U})\right), \boldsymbol{\theta}_{0}=\left(\theta_{0}^{1}, \ldots, \theta_{0}^{m}\right)$, where $\mathbf{U}=\left(U^{1}, \ldots, U^{N}\right)$ are the parameters of a solution. We will require also that all the functions $\Phi^{i}(\boldsymbol{\theta}, \mathbf{U})$ are $2 \pi$-periodic with respect to each $\theta^{\alpha}, \alpha=1, \ldots, m$.

\footnotetext{
*This paper is a contribution to the Special Issue "Geometrical Methods in Mathematical Physics". The full collection is available at http://www.emis.de/journals/SIGMA/GMMP2012.html
} 
Consider a set $\Lambda$ of functions $\boldsymbol{\Phi}\left(\boldsymbol{\theta}+\boldsymbol{\theta}_{0}, \mathbf{U}\right)$, depending smoothly on the parameters $\mathbf{U}$ and satisfying system (1.3) for all $\mathbf{U}$.

In the Whitham approach the parameters $\mathbf{U}$ and $\boldsymbol{\theta}_{0}$ become slowly varying functions of $x$ and $t, \mathbf{U}=\mathbf{U}(X, T), \boldsymbol{\theta}_{0}=\boldsymbol{\theta}_{0}(X, T)$, where $X=\epsilon x, T=\epsilon t(\epsilon \rightarrow 0)$.

For the construction of the corresponding asymptotic solution the functions $\mathbf{U}(X, T)$ must satisfy some system of differential equations (the Whitham system). In the simplest case (see [31]), we try to find asymptotic solutions

$$
\varphi^{i}(\boldsymbol{\theta}, X, T)=\sum_{k \geq 0} \Psi_{(k)}^{i}\left(\frac{\mathbf{S}(X, T)}{\epsilon}+\boldsymbol{\theta}, X, T\right) \epsilon^{k}
$$

with $2 \pi$-periodic in $\boldsymbol{\theta}$ functions $\boldsymbol{\Psi}_{(k)}$ satisfying system (1.1), i.e.

$$
F^{i}\left(\boldsymbol{\varphi}, \epsilon \boldsymbol{\varphi}_{T}, \epsilon \boldsymbol{\varphi}_{X}, \ldots\right)=0, \quad i=1, \ldots, n .
$$

The function $\mathbf{S}(X, T)=\left(S^{1}(X, T), \ldots, S^{m}(X, T)\right)$ is called the "modulated phase" of solution (1.4).

Assume now that the function $\boldsymbol{\Psi}_{(0)}(\boldsymbol{\theta}, X, T)$ belongs to the family $\Lambda$ of $m$-phase solutions of (1.1) for all $X$ and $T$. We have then

$$
\boldsymbol{\Psi}_{(0)}(\boldsymbol{\theta}, X, T)=\boldsymbol{\Phi}\left(\boldsymbol{\theta}+\boldsymbol{\theta}_{0}(X, T), \mathbf{U}(X, T)\right),
$$

and

$$
S_{T}^{\alpha}(X, T)=\omega^{\alpha}(\mathbf{U}(X, T)), \quad S_{X}^{\alpha}(X, T)=k^{\alpha}(\mathbf{U}(X, T)),
$$

as follows after the substitution of (1.4) into system (1.1).

In the simplest case the functions $\boldsymbol{\Psi}_{(k)}(\boldsymbol{\theta}, X, T)$ are determined from the linear systems

$$
\hat{L}_{j\left[\mathbf{U}, \boldsymbol{\theta}_{0}\right]}^{i}(X, T) \Psi_{(k)}^{j}(\boldsymbol{\theta}, X, T)=f_{(k)}^{i}(\boldsymbol{\theta}, X, T),
$$

where $\hat{L}_{j\left[\mathbf{U}, \boldsymbol{\theta}_{0}\right]}^{i}(X, T)$ is a linear operator defined by the linearization of system (1.3) on the solution (1.5). The resolvability conditions of systems (1.6) in the space of periodic functions can be written as the conditions of orthogonality of the functions $\mathbf{f}_{(k)}(\boldsymbol{\theta}, X, T)$ to all the "left eigenvectors" (the eigenvectors of the adjoint operator) of the operator $\hat{L}_{j\left[\mathbf{U}, \boldsymbol{\theta}_{0}\right]}^{i}(X, T)$ corresponding to zero eigenvalue.

We should say, however, that the resolvability conditions of systems (1.6) can actually be quite complicated in general multi-phase case, since the eigenspaces of the operators $\hat{L}_{\left[\mathbf{U}, \boldsymbol{\theta}_{0}\right]}$ and $\hat{L}_{\left[\mathbf{U}, \boldsymbol{\theta}_{0}\right]}^{\dagger}$ on the space of $2 \pi$-periodic functions can be rather nontrivial in the multi-phase situation. Thus, even the dimension of the kernels of $\hat{L}_{\left[\mathbf{U}, \boldsymbol{\theta}_{0}\right]}$ and $\hat{L}_{\left[\mathbf{U}, \boldsymbol{\theta}_{0}\right]}^{\dagger}$ can depend in a highly nontrivial way on the values of $\mathbf{U}$. In general, the picture arising in the $\mathbf{U}$-space can be rather complicated. As a result, the determination of the next corrections from systems (1.6) is impossible in general multiphase situation and the corrections to the main approximation (1.5) have more complicated and rather nontrivial form [4-6].

These difficulties do not arise commonly in the single-phase situation $(m=1)$ where the behavior of eigenvectors of $\hat{L}_{\left[\mathbf{U}, \boldsymbol{\theta}_{0}\right]}$ and $\hat{L}_{\left[\mathbf{U}, \boldsymbol{\theta}_{0}\right]}^{\dagger}$, as a rule, is quite regular. The resolvability conditions of system (1.6) for $k=1$

$$
\hat{L}_{j\left[\mathbf{U}, \boldsymbol{\theta}_{0}\right]}^{i}(X, T) \Psi_{(1)}^{j}(\boldsymbol{\theta}, X, T)=f_{(1)}^{i}(\boldsymbol{\theta}, X, T)
$$

with relations $k_{T}=\omega_{X}$ define in this case the Whitham system for the single-phase solutions of (1.1) which plays the central role in considering the slow modulations. 
For the multi-phase solutions the Whitham system is usually given by the orthogonality conditions of the right-hand part of (1.7) to the maximal set of "regular" left eigenvectors corresponding to zero eigenvalues which are defined for all values of $\mathbf{U}$ and depend smoothly on $\mathbf{U}$. As a rule, this system is equivalent to the conditions obtained by the averaging of some complete set of conservation laws of system (1.1) having the local form

$$
P_{t}^{\nu}\left(\boldsymbol{\varphi}, \boldsymbol{\varphi}_{t}, \boldsymbol{\varphi}_{x}, \ldots\right)=Q_{x}^{\nu}\left(\boldsymbol{\varphi}, \boldsymbol{\varphi}_{t}, \boldsymbol{\varphi}_{x}, \ldots\right), \quad \nu=1, \ldots, N
$$

The Whitham system is written usually as a system of hydrodynamic type

$$
U_{T}^{\nu}=V_{\mu}^{\nu}(\mathbf{U}) U_{X}^{\mu}
$$

and gives the main approximation for the connection between spatial and time derivatives of the parameters $U^{\nu}(X, T)$. The variables $T$ and $X$ represent the "slow" variables $T=\epsilon t, X=\epsilon x$, connected with the variables $t$ and $x$ by a small parameter $\epsilon$. Thus, the Whitham system (1.8) represents a homogeneous quasi-linear system of hydrodynamic type connecting the derivatives of slow modulated parameters.

As mentioned above, the construction of the asymptotic series (1.4) in the multi-phase case is impossible in general situation (see [4-6]). Nevertheless, the Whitham system (1.8) and the leading term of the expansion (1.4) play the major role in consideration of modulated solutions also in this case, representing the main approximation for corresponding modulated solutions. The corrections to the main term have in general more nontrivial form than (1.4), but they also tend to zero in the limit $\epsilon \rightarrow 0$ [4-6].

Let us give here just some incomplete list of the classical papers devoted to the foundations of the Whitham method $[1,4-8,14-16,22-25,28,29,31,39,40,42,48-50]$. We will be interested here only in Hamiltonian aspects of the Whitham method. In the remaining part of the Introduction we will give the definition of the "regular" Whitham system for the complete regular family of $m$-phase solutions which will be used everywhere below.

Let us use for simplicity the notation $\Lambda$ both for the family of the functions $\boldsymbol{\Phi}\left(\boldsymbol{\theta}+\boldsymbol{\theta}_{0}, \mathbf{U}\right)$ and the corresponding family of $m$-phase solutions of system (1.1), such that we will denote by $\Lambda$ both the families of the functions $\boldsymbol{\Phi}\left(\boldsymbol{\theta}+\boldsymbol{\theta}_{0}, \mathbf{U}\right)$ in the space of $2 \pi$-periodic in all $\theta^{\alpha}$ functions $\boldsymbol{\varphi}(\boldsymbol{\theta})$ and $\boldsymbol{\varphi}_{\left[\mathbf{U}, \boldsymbol{\theta}_{0}\right]}(x)=\boldsymbol{\Phi}\left(\mathbf{k}(\mathbf{U}) x+\boldsymbol{\theta}_{0}, \mathbf{U}\right)$. We will assume everywhere below that the family $\Lambda$ represents a smooth family of $m$-phase solutions of system (1.1) in the sense discussed above.

It is generally assumed that the parameters $k^{\alpha}, \omega^{\alpha}$ are independent on the family $\Lambda$, such that the full family of the $m$-phase solutions of (1.1) depends on $N=2 m+s(s \geq 0)$ parameters $U^{\nu}$ and $m$ initials phase shifts $\theta_{0}^{\alpha}$. In this case it is convenient to represent the parameters $\mathbf{U}$ in the form $\mathbf{U}=(\mathbf{k}, \boldsymbol{\omega}, \mathbf{n})$, where $\mathbf{k}$ represents the wave numbers, $\boldsymbol{\omega}$ are the frequencies of the $m$-phase solutions and $\mathbf{n}=\left(n^{1}, \ldots, n^{s}\right)$ are some additional parameters (if any).

It is easy to see that the functions $\boldsymbol{\Phi}_{\theta^{\alpha}}\left(\boldsymbol{\theta}+\boldsymbol{\theta}_{0}, \mathbf{k}, \boldsymbol{\omega}, \mathbf{n}\right), \alpha=1, \ldots, m, \boldsymbol{\Phi}_{n^{l}}\left(\boldsymbol{\theta}+\boldsymbol{\theta}_{0}, \mathbf{k}, \boldsymbol{\omega}, \mathbf{n}\right)$, $l=1, \ldots, s$, belong to the kernel of the operator $\hat{L}_{j\left[\mathbf{k}, \boldsymbol{\omega}, \mathbf{n}, \boldsymbol{\theta}_{0}\right]}^{i}$. In the regular case it is natural to assume that the set of the functions $\left(\boldsymbol{\Phi}_{\theta^{\alpha}}, \boldsymbol{\Phi}_{n^{l}}\right)$ represents the maximal linearly independent set of the kernel vectors of the operator $\hat{L}$ regularly depending on the parameters $(\mathbf{k}, \boldsymbol{\omega}, \mathbf{n})$. For the construction of the "regular" Whitham system we have to require the following property of regularity and completeness of the family of $m$-phase solutions of system (1.1).

Definition 1.1. We call a family $\Lambda$ a complete regular family of $m$-phase solutions of system (1.1) if:

1) the values $\mathbf{k}=\left(k^{1}, \ldots, k^{m}\right), \boldsymbol{\omega}=\left(\omega^{1}, \ldots, \omega^{m}\right)$ represent independent parameters on the family $\Lambda$, such that the total set of parameters of the $m$-phase solutions can be represented in the form $\left(\mathbf{U}, \boldsymbol{\theta}_{0}\right)=\left(\mathbf{k}, \boldsymbol{\omega}, \mathbf{n}, \boldsymbol{\theta}_{0}\right)$; 
2) the functions $\boldsymbol{\Phi}_{\theta^{\alpha}}\left(\boldsymbol{\theta}+\boldsymbol{\theta}_{0}, \mathbf{k}, \boldsymbol{\omega}, \mathbf{n}\right), \boldsymbol{\Phi}_{n^{l}}\left(\boldsymbol{\theta}+\boldsymbol{\theta}_{0}, \mathbf{k}, \boldsymbol{\omega}, \mathbf{n}\right)$ are linearly independent and give the maximal linearly independent set among the kernel vectors of the operator $\hat{L}_{j\left[\mathbf{k}, \boldsymbol{\omega}, \mathbf{n}, \boldsymbol{\theta}_{0}\right]}^{i}$, smoothly depending on the parameters $(\mathbf{k}, \boldsymbol{\omega}, \mathbf{n})$ on the whole set of parameters;

3) the operator $\hat{L}_{j\left[\mathbf{k}, \boldsymbol{\omega}, \mathbf{n}, \boldsymbol{\theta}_{0}\right]}^{i}$ has exactly $m+s$ linearly independent left eigenvectors with zero eigenvalue

$$
\boldsymbol{\kappa}_{[\mathbf{U}]}^{(q)}\left(\boldsymbol{\theta}+\boldsymbol{\theta}_{0}\right)=\boldsymbol{\kappa}_{[\mathbf{k}, \boldsymbol{\omega}, \mathbf{n}]}^{(q)}\left(\boldsymbol{\theta}+\boldsymbol{\theta}_{0}\right), \quad q=1, \ldots, m+s,
$$

among the vectors smoothly depending on the parameters $(\mathbf{k}, \boldsymbol{\omega}, \mathbf{n})$ on the whole set of parameters.

By definition, we will call the regular Whitham system for a complete regular family of $m$-phase solutions of (1.1) the conditions of orthogonality of the discrepancy $\mathbf{f}_{(1)}(\boldsymbol{\theta}, X, T)$ to the functions $\boldsymbol{\kappa}_{[\mathbf{U}(X, T)]}^{(q)}\left(\boldsymbol{\theta}+\boldsymbol{\theta}_{0}(X, T)\right)$

$$
\int_{0}^{2 \pi} \cdots \int_{0}^{2 \pi} \kappa_{[\mathbf{U}(X, T)] i}^{(q)}\left(\boldsymbol{\theta}+\boldsymbol{\theta}_{0}(X, T)\right) f_{(1)}^{i}(\boldsymbol{\theta}, X, T) \frac{\mathrm{d}^{m} \theta}{(2 \pi)^{m}}=0, \quad q=1, \ldots, m+s,
$$

with the compatibility conditions

$$
k_{T}^{\alpha}=\omega_{X}^{\alpha}
$$

System (1.9), (1.10) gives $m+(m+s)=2 m+s=N$ conditions at every $X$ and $T$ for the parameters of the zero approximation $\Psi_{(0)}(\boldsymbol{\theta}, X, T)$.

It is well known that the Whitham system does not include the parameters $\theta_{0}^{\alpha}(X, T)$ and provides restrictions only to the parameters $U^{\nu}(X, T)$ of the zero approximation. Let us prove here a simple lemma which confirms this property under the conditions formulated above ${ }^{1}$.

Lemma 1.1. Under the regularity conditions formulated above the orthogonality conditions (1.9) do not contain the functions $\theta_{0}^{\alpha}(X, T)$ and give constraints only to the functions $U^{\nu}(X, T)$, having the form

$$
C_{\nu}^{(q)}(\mathbf{U}) U_{T}^{\nu}-D_{\nu}^{(q)}(\mathbf{U}) U_{X}^{\nu}=0, \quad q=1, \ldots, m+s,
$$

with some functions $C_{\nu}^{(q)}(\mathbf{U}), D_{\nu}^{(q)}(\mathbf{U})$.

Proof. Let us write down the part $\mathbf{f}_{(1)}^{\prime}$ of the function $\mathbf{f}_{(1)}$, which contains the derivatives $\theta_{0 T}^{\beta}(X, T)$ and $\theta_{0 X}^{\beta}(X, T)$. We have

$$
\begin{aligned}
f_{(1)}^{\prime i}(\boldsymbol{\theta}, X, T)= & -\frac{\partial F^{i}}{\partial \varphi_{t}^{j}}\left(\mathbf{\Psi}_{(0)}, \ldots\right) \Psi_{(0) \theta^{\beta}}^{j} \theta_{0 T}^{\beta}-\frac{\partial F^{i}}{\partial \varphi_{x}^{j}}\left(\boldsymbol{\Psi}_{(0)}, \ldots\right) \Psi_{(0) \theta^{\beta}}^{j} \theta_{0 X}^{\beta} \\
& -\frac{\partial F^{i}}{\partial \varphi_{t t}^{j}}\left(\Psi_{(0)}, \ldots\right) 2 \omega^{\alpha}(X, T) \Psi_{(0) \theta^{\alpha} \theta^{\beta}}^{j} \theta_{0 T}^{\beta} \\
& -\frac{\partial F^{i}}{\partial \varphi_{x x}^{j}}\left(\Psi_{(0)}, \ldots\right) 2 k^{\alpha}(X, T) \Psi_{(0) \theta^{\alpha} \theta^{\beta}}^{j} \theta_{0 X}^{\beta}-\cdots
\end{aligned}
$$

\footnotetext{
${ }^{1}$ This simple fact was present in the Whitham approach from the very beginning (see [31,48-50]). In fact, under various assumptions it can be also shown that the additional phase shifts $\theta_{0}^{\alpha}(X, T)$ can be always absorbed by the functions $S^{\alpha}(X, T)$ after a suitable correction of initial data (see, e.g., [9, 23, 24,35]). It should be noted, however, that the corresponding phase shift can play rather important role in the weakly nonlocal case [39] (see also $[9,33])$.
} 
Let us choose the parameters $\mathbf{U}$ in the form

$$
\mathbf{U}=\left(k^{1}, \ldots, k^{m}, \omega^{1}, \ldots, \omega^{m}, n^{1}, \ldots, n^{s}\right) .
$$

We can write then

$$
\begin{aligned}
f_{(1)}^{\prime i}(\boldsymbol{\theta}, X, T)= & {\left[-\frac{\partial}{\partial \omega^{\beta}} F^{i}\left(\boldsymbol{\Phi}\left(\boldsymbol{\theta}+\boldsymbol{\theta}_{0}, \mathbf{U}\right), \ldots\right)+\hat{L}_{j}^{i} \frac{\partial}{\partial \omega^{\beta}} \Phi^{j}\left(\boldsymbol{\theta}+\boldsymbol{\theta}_{0}, \mathbf{U}\right)\right] \theta_{0 T}^{\beta} } \\
& +\left[-\frac{\partial}{\partial k^{\beta}} F^{i}\left(\boldsymbol{\Phi}\left(\boldsymbol{\theta}+\boldsymbol{\theta}_{0}, \mathbf{U}\right), \ldots\right)+\hat{L}_{j}^{i} \frac{\partial}{\partial k^{\beta}} \Phi^{j}\left(\boldsymbol{\theta}+\boldsymbol{\theta}_{0}, \mathbf{U}\right)\right] \theta_{0 X}^{\beta} .
\end{aligned}
$$

The total derivatives $\partial F^{i} / \partial \omega^{\beta}$ and $\partial F^{i} / \partial k^{\beta}$ are identically equal to zero on $\Lambda$ according to (1.3). We have then

$$
\int_{0}^{2 \pi} \cdots \int_{0}^{2 \pi} \kappa_{[\mathbf{U}(X, T)] i}^{(q)}\left(\boldsymbol{\theta}+\boldsymbol{\theta}_{0}(X, T)\right) f_{(1)}^{\prime i}(\boldsymbol{\theta}, X, T) \frac{\mathrm{d}^{m} \theta}{(2 \pi)^{m}} \equiv 0
$$

since $\boldsymbol{\kappa}_{[\mathbf{U}(X, T)]}^{(q)}\left(\boldsymbol{\theta}+\boldsymbol{\theta}_{0}(X, T)\right)$ are left eigenvectors of $\hat{L}$ with the zero eigenvalues.

It is not difficult to see also that all the $\boldsymbol{\theta}_{0}(X, T)$ in the arguments of $\boldsymbol{\Phi}$ and $\boldsymbol{\kappa}^{(q)}$ disappear after the integration with respect to $\boldsymbol{\theta}$, so we get the statement of the lemma.

We can claim then that the regular Whitham system has the following general form

$$
\begin{aligned}
& \frac{\partial k^{\alpha}}{\partial U^{\nu}} U_{T}^{\nu}=\frac{\partial \omega^{\alpha}}{\partial U^{\nu}} U_{X}^{\nu}, \quad \alpha=1, \ldots, m, \\
& C_{\nu}^{(q)}(\mathbf{U}) U_{T}^{\nu}=D_{\nu}^{(q)}(\mathbf{U}) U_{X}^{\nu}, \quad q=1, \ldots, m+s .
\end{aligned}
$$

Let us note that according to our assumptions we have here rank $\left\|\partial k^{\alpha} / \partial U^{\nu}\right\|=m$. In generic case the derivatives $U_{T}^{\nu}$ can be expressed in terms of $U_{X}^{\mu}$ and the Whitham system can be written in the form (1.8).

\section{Lagrangian and Hamiltonian formulations of the Whitham method}

Together with the formulation of Whitham's method the Lagrangian structure of the equations of slow modulations was proposed [48-50]. The method of averaging of Lagrangian function introduced by Whitham can be formulated in the following way. We assume that the original system (1.1) is lagrangian with the local action of the form

$$
S=\iint L\left(\boldsymbol{\varphi}, \boldsymbol{\varphi}_{t}, \boldsymbol{\varphi}_{x}, \boldsymbol{\varphi}_{t t}, \boldsymbol{\varphi}_{x t}, \boldsymbol{\varphi}_{x x}, \ldots\right) \mathrm{d} x \mathrm{~d} t,
$$

such that the functions $F^{i}$ have the form

$$
F^{i}\left(\boldsymbol{\varphi}, \boldsymbol{\varphi}_{t}, \boldsymbol{\varphi}_{x}, \ldots\right)=\frac{\delta S}{\delta \varphi^{i}(x, t)}=\frac{\partial L}{\partial \varphi^{i}}-\frac{\partial}{\partial t} \frac{\partial L}{\partial \varphi_{t}^{i}}-\frac{\partial}{\partial x} \frac{\partial L}{\partial \varphi_{x}^{i}}+\cdots
$$

Let us assume here for simplicity that the parameters $(\mathbf{k}, \boldsymbol{\omega})=\left(k^{1}, \ldots, k^{m}, \omega^{1}, \ldots, \omega^{m}\right)$ give the complete set of independent parameters on the family of $m$-phase solutions (excluding the initial phase shifts), such that the number of parameters $U^{\nu}$ is equal to $2 \mathrm{~m}$.

The linearized operator $\hat{L}_{j\left[\mathbf{k}, \boldsymbol{\omega}, \boldsymbol{\theta}_{0}\right]}^{i}(X, T)$ in (1.6) is given now by the distribution

$$
L_{j\left[\mathbf{k}, \boldsymbol{\omega}, \boldsymbol{\theta}_{0}\right]}^{i}\left(\boldsymbol{\theta}, \boldsymbol{\theta}^{\prime}\right)=\frac{\delta^{2} S}{\delta \Phi^{i}(\boldsymbol{\theta}) \delta \Phi^{j}\left(\boldsymbol{\theta}^{\prime}\right)},
$$


where

$$
S=\int_{0}^{2 \pi} \cdots \int_{0}^{2 \pi} L\left(\boldsymbol{\Phi}, \omega^{\alpha} \boldsymbol{\Phi}_{\theta^{\alpha}}, k^{\beta} \boldsymbol{\Phi}_{\theta^{\beta}}, \ldots\right) \frac{\mathrm{d}^{m} \theta}{(2 \pi)^{m}}
$$

is a self-adjoint operator.

Throughout the paper we will always understand the integration with respect to $\boldsymbol{\theta}$ as the averaging procedure. For this reason, all the integrals over $\mathrm{d}^{m} \theta$ will be defined with the factor $1 /(2 \pi)^{m}$. In particular, we will also assume that the variational derivatives of the type $\delta S / \delta \varphi^{i}(\boldsymbol{\theta})$ are defined as

$$
\delta S \equiv \int_{0}^{2 \pi} \cdots \int_{0}^{2 \pi} \frac{\delta S}{\delta \varphi^{i}(\boldsymbol{\theta})} \delta \varphi^{i}(\boldsymbol{\theta}) \frac{\mathrm{d}^{m} \theta}{(2 \pi)^{m}}
$$

on the space of $2 \pi$-periodic in $\boldsymbol{\theta}$ functions.

We also define here the delta function $\delta\left(\boldsymbol{\theta}-\boldsymbol{\theta}^{\prime}\right)$ and its higher derivatives $\delta_{\theta^{\alpha_{1}} \ldots \theta^{\alpha_{s}}}\left(\boldsymbol{\theta}-\boldsymbol{\theta}^{\prime}\right)$ on the space of $2 \pi$-periodic functions by the formula

$$
\int_{0}^{2 \pi} \cdots \int_{0}^{2 \pi} \delta_{\theta^{\alpha_{1} \ldots \theta^{\alpha_{s}}}}\left(\boldsymbol{\theta}-\boldsymbol{\theta}^{\prime}\right) \psi\left(\boldsymbol{\theta}^{\prime}\right) \frac{\mathrm{d}^{m} \theta^{\prime}}{(2 \pi)^{m}} \equiv \psi_{\theta^{\alpha_{1} \ldots \theta^{\alpha_{s}}}}(\boldsymbol{\theta}) .
$$

The functions $\boldsymbol{\Phi}_{\theta^{\alpha}}, \alpha=1, \ldots, m$, represent both the left and the right eigenfunctions of the operator $\hat{L}_{j\left[\mathbf{k}, \boldsymbol{\omega}, \boldsymbol{\theta}_{0}\right]}^{i}(X, T)$, corresponding to the zero eigenvalue.

Under the assumption that the family of the $m$-phase solutions $\Lambda$ is a complete regular family of $m$-phase solutions of (1.1) we assume that the functions $\boldsymbol{\Phi}_{\theta^{\alpha}}\left(\boldsymbol{\theta}+\boldsymbol{\theta}_{0}, \mathbf{k}, \boldsymbol{\omega}\right)$ are linearly independent and give the maximal linearly independent set among the kernel vectors of the operator $\hat{L}_{j\left[\mathbf{k}, \boldsymbol{\omega}, \boldsymbol{\theta}_{0}\right]}^{i}$ smoothly depending on the parameters $(\mathbf{k}, \boldsymbol{\omega})$. The regular Whitham system is given then by the conditions $k_{T}^{\alpha}=\omega_{X}^{\alpha}$ and $m$ conditions of orthogonality of the function $\mathbf{f}_{(1)}(\boldsymbol{\theta}, X, T)$ to the functions $\boldsymbol{\Phi}_{\theta^{\alpha}}\left(\boldsymbol{\theta}+\boldsymbol{\theta}_{0}, \mathbf{k}, \boldsymbol{\omega}\right)$.

According to the Whitham procedure the Whitham system on the parameters $(\mathbf{k}, \boldsymbol{\omega})$ is obtained from the condition of extremality of the action

$$
\Sigma^{(0)}[\mathbf{S}]=\iiint_{0}^{2 \pi} \cdots \int_{0}^{2 \pi} L\left(\mathbf{\Phi}, S_{T}^{\alpha} \boldsymbol{\Phi}_{\theta^{\alpha}}, S_{X}^{\beta} \boldsymbol{\Phi}_{\theta^{\beta}}, \ldots\right) \frac{\mathrm{d}^{m} \theta}{(2 \pi)^{m}} \mathrm{~d} X \mathrm{~d} T
$$

under the conditions $k^{\alpha}=S_{X}^{\alpha}, \omega^{\alpha}=S_{T}^{\alpha}$.

The conditions $k_{T}^{\alpha}=\omega_{X}^{\alpha}$ and $\delta \Sigma / \delta S^{\alpha}(X, T)=0$ give a system of $2 m$ equations on the parameters $(\mathbf{k}, \boldsymbol{\omega})$.

It is not difficult to see that the system given by the variation of the "averaged" action coincides with the conditions of orthogonality of the function $\mathbf{f}_{(1)}(\boldsymbol{\theta}, X, T)$ to the functions $\boldsymbol{\Phi}_{\theta^{\alpha}}\left(\boldsymbol{\theta}+\boldsymbol{\theta}_{0}, \mathbf{k}, \boldsymbol{\omega}\right)$. Indeed, let us consider the action

$$
\begin{aligned}
\Sigma[\mathbf{S}, \boldsymbol{\varphi}, \epsilon] & =\int L\left(\boldsymbol{\varphi}\left(\frac{\mathbf{S}(X, T)}{\epsilon}+\boldsymbol{\theta}, X, T\right), \epsilon \frac{\partial}{\partial T} \boldsymbol{\varphi}\left(\frac{\mathbf{S}(X, T)}{\epsilon}+\boldsymbol{\theta}, X, T\right), \ldots\right) \frac{\mathrm{d}^{m} \theta}{(2 \pi)^{m}} \mathrm{~d} X \mathrm{~d} T \\
& =\Sigma^{(0)}[\mathbf{S}, \boldsymbol{\varphi}]+\epsilon \Sigma^{(1)}[\mathbf{S}, \boldsymbol{\varphi}]+\epsilon^{2} \Sigma^{(2)}[\mathbf{S}, \boldsymbol{\varphi}]+\cdots
\end{aligned}
$$

defined on the functions $\boldsymbol{\varphi}(\boldsymbol{\theta}, X, T), 2 \pi$-periodic in each $\theta^{\alpha}$. Taking into account the relation

$$
\frac{\delta \Sigma}{\delta S^{\alpha}(X, T)}=\epsilon^{-1} \int_{0}^{2 \pi} \cdots \int_{0}^{2 \pi} \varphi_{\theta^{\alpha}}^{i}(\boldsymbol{\theta}, X, T) \frac{\delta \Sigma}{\delta \varphi^{i}(\boldsymbol{\theta}, X, T)} \frac{\mathrm{d}^{m} \theta}{(2 \pi)^{m}}
$$

and the invariance of the action with respect to the shifts

$$
\mathbf{S}(X, T) \rightarrow \mathbf{S}(X, T)+\Delta \mathbf{S}
$$


it is easy to see that

$$
\begin{aligned}
& \int_{0}^{2 \pi} \cdots \int_{0}^{2 \pi} \varphi_{\theta^{\alpha}}^{i}(\boldsymbol{\theta}, X, T) \frac{\delta \Sigma^{(0)}}{\delta \varphi^{i}(\boldsymbol{\theta}, X, T)} \frac{\mathrm{d}^{m} \theta}{(2 \pi)^{m}} \equiv 0 \\
& \frac{\delta \Sigma^{(0)}}{\delta S^{\alpha}(X, T)}=\int_{0}^{2 \pi} \cdots \int_{0}^{2 \pi} \varphi_{\theta^{\alpha}}^{i}(\boldsymbol{\theta}, X, T) \frac{\delta \Sigma^{(1)}}{\delta \varphi^{i}(\boldsymbol{\theta}, X, T)} \frac{\mathrm{d}^{m} \theta}{(2 \pi)^{m}}
\end{aligned}
$$

etc.

Substituting the functions $\boldsymbol{\varphi}(\boldsymbol{\theta}, X, T)$ in the form $\boldsymbol{\varphi}(\boldsymbol{\theta}, X, T)=\boldsymbol{\Phi}\left(\boldsymbol{\theta}+\boldsymbol{\theta}_{0}, \mathbf{S}_{X}, \mathbf{S}_{T}\right)$ in the relations above, we can see that we have to include now the additional dependence of the functions $\boldsymbol{\varphi}(\boldsymbol{\theta}, X, T)$ on $\mathbf{S}_{X}$ and $\mathbf{S}_{T}$ in relation (2.2). However, due to the relation

$$
\frac{\delta \Sigma^{(0)}}{\delta \varphi^{i}(\boldsymbol{\theta}, X, T)} \equiv 0
$$

on the family $\Lambda$, the relation (2.2) will not change in this situation. Taking also into account the equality

$$
f_{(1)}^{i}(\boldsymbol{\theta}, X, T)=\frac{\delta \Sigma^{(1)}}{\delta \varphi^{i}(\boldsymbol{\theta}, X, T)}
$$

we get the required statement.

Under the assumption of the completeness and regularity of the family $\Lambda$ we can see then that the averaged action (2.1) defines a lagrangian structure of the regular Whitham system in general multiphase case. We should say also that the cases with additional parameters $\mathbf{n}$, as a rule, can be also included into the scheme described above with the aid of the Whitham "pseudo-phases" [49]. Let us note also that different questions connected with the justification of the averaging of Lagrangian functions in different orders can be found in [7].

Another approach to the construction of the regular Whitham system is connected with the method of averaging of conservation laws. According to further consideration of the Hamiltonian structure of the Whitham equations we will assume now that system (1.1) is written in an evolutionary form

$$
\varphi_{t}^{i}=F^{i}\left(\boldsymbol{\varphi}, \boldsymbol{\varphi}_{x}, \boldsymbol{\varphi}_{x x}, \ldots\right) .
$$

The families of the $m$-phase solutions of (2.3) are defined then by solutions of the system

$$
\omega^{\alpha} \varphi_{\theta^{\alpha}}^{i}=F^{i}\left(\boldsymbol{\varphi}, k^{\beta} \varphi_{\theta^{\beta}}, \ldots\right)
$$

on the space of $2 \pi$-periodic in each $\theta^{\alpha}$ functions $\varphi(\theta)$.

We will assume that the conservation laws of system (2.3) have the form

$$
P_{t}^{\nu}\left(\boldsymbol{\varphi}, \boldsymbol{\varphi}_{x}, \boldsymbol{\varphi}_{x x}, \ldots\right)=Q_{x}^{\nu}\left(\boldsymbol{\varphi}, \boldsymbol{\varphi}_{x}, \boldsymbol{\varphi}_{x x}, \ldots\right),
$$

such that the values

$$
I^{\nu}=\int_{-\infty}^{+\infty} P^{\nu}\left(\boldsymbol{\varphi}, \boldsymbol{\varphi}_{x}, \boldsymbol{\varphi}_{x x}, \ldots\right) \mathrm{d} x
$$

represent translationally invariant conservative quantities for the system (2.3) in the case of the rapidly decreasing at infinity functions $\varphi(x)$. We can also define the conservation laws for system (2.3) in the periodic case with a fixed period $K$

$$
I^{\nu}=\frac{1}{K} \int_{0}^{K} P^{\nu}\left(\boldsymbol{\varphi}, \boldsymbol{\varphi}_{x}, \boldsymbol{\varphi}_{x x}, \ldots\right) \mathrm{d} x
$$


or in the quasiperiodic case

$$
I^{\nu}=\lim _{K \rightarrow \infty} \frac{1}{2 K} \int_{-K}^{K} P^{\nu}\left(\boldsymbol{\varphi}, \boldsymbol{\varphi}_{x}, \boldsymbol{\varphi}_{x x}, \ldots\right) \mathrm{d} x .
$$

It is natural also to define the variational derivatives of the functionals $I^{\nu}$ with respect to the variations of $\varphi(x)$ having the same periodic or quasiperiodic properties as the original functions. Easy to see then that the standard Euler-Lagrange expressions for the variational derivatives can be used in this case.

Let us write the functionals $I^{\nu}$ in the general form

$$
I^{\nu}=\int P^{\nu}\left(\varphi, \varphi_{x}, \varphi_{x x}, \ldots\right) \mathrm{d} x
$$

assuming the appropriate definition in the corresponding situations.

Let us define a quasiperiodic function $\varphi(x)$ with fixed quasiperiods $\left(k^{1}, \ldots, k^{m}\right)$ as a smooth periodic function $\boldsymbol{\varphi}(\boldsymbol{\theta})$ on the torus $\mathbb{T}^{m}$ which is restricted on the corresponding straight-line winding

$$
\varphi\left(\mathbf{k} x+\theta_{0}\right) \rightarrow \varphi(x) .
$$

Let us define the functionals

$$
J^{\nu}=\int_{0}^{2 \pi} \cdots \int_{0}^{2 \pi} P^{\nu}\left(\boldsymbol{\varphi}, k^{\beta} \boldsymbol{\varphi}_{\theta^{\beta}}, \ldots\right) \frac{\mathrm{d}^{m} \theta}{(2 \pi)^{m}}
$$

on the space of $2 \pi$-periodic in $\boldsymbol{\theta}$ functions.

It's not difficult to see that the functions

$$
\zeta_{i[\mathbf{U}]}^{(\nu)}\left(\boldsymbol{\theta}+\boldsymbol{\theta}_{0}\right)=\left.\frac{\delta J^{\nu}}{\delta \varphi^{i}(\boldsymbol{\theta})}\right|_{\boldsymbol{\varphi}(\boldsymbol{\theta})=\mathbf{\Phi}\left(\boldsymbol{\theta}+\boldsymbol{\theta}_{0}, \mathbf{U}\right)}
$$

represent left eigenvectors of the operator $\hat{L}_{j\left[\mathbf{U}, \boldsymbol{\theta}_{0}\right]}^{i}$ with zero eigenvalues regularly depending on parameters $\mathbf{U}$ on a fixed smooth family $\Lambda$.

Indeed, the operator $\hat{L}_{j\left[\mathbf{U}, \boldsymbol{\theta}_{0}\right]}^{i}$ is defined in this case by the distribution

$$
L_{j\left[\mathbf{U}, \boldsymbol{\theta}_{0}\right]}^{i}\left(\boldsymbol{\theta}, \boldsymbol{\theta}^{\prime}\right)=\delta_{j}^{i} \omega^{\alpha} \delta_{\theta^{\alpha}}\left(\boldsymbol{\theta}-\boldsymbol{\theta}^{\prime}\right)-\left.\frac{\delta F^{i}\left(\boldsymbol{\varphi}, k^{\beta} \boldsymbol{\varphi}_{\theta^{\beta}}, \ldots\right)}{\delta \varphi^{j}\left(\boldsymbol{\theta}^{\prime}\right)}\right|_{\boldsymbol{\varphi}(\boldsymbol{\theta})=\boldsymbol{\Phi}\left(\boldsymbol{\theta}+\boldsymbol{\theta}_{0}, \mathbf{U}\right)} .
$$

We have

$$
\int_{0}^{2 \pi} \cdots \int_{0}^{2 \pi} \frac{\delta J^{\nu}}{\delta \varphi^{i}(\boldsymbol{\theta})}\left(\omega^{\alpha} \varphi_{\theta^{\alpha}}^{i}-F^{i}\left(\boldsymbol{\varphi}, k^{\beta} \boldsymbol{\varphi}_{\theta^{\beta}}, \ldots\right)\right) \frac{\mathrm{d}^{m} \theta}{(2 \pi)^{m}} \equiv 0
$$

for any translationally invariant integral of (2.3). Taking the variational derivative of this relation with respect to $\varphi^{j}\left(\boldsymbol{\theta}^{\prime}\right)$ on $\Lambda$ we get the required statement.

Thus, we can write

$$
\zeta_{i[\mathbf{U}]}^{(\nu)}(\boldsymbol{\theta})=\sum_{q} c_{q}^{\nu}(\mathbf{U}) \kappa_{i[\mathbf{U}]}^{(q)}(\boldsymbol{\theta})
$$

with some smooth functions $c_{q}^{\nu}(\mathbf{U})$ on a complete regular family $\Lambda$.

For the construction of the regular Whitham system on a complete regular family of $m$-phase solutions of (2.3) we need a sufficient number of the first integrals (2.5) such that the values of the functionals $J^{\nu}$ on $\Lambda$ represent the full set of parameters $U^{\nu}=\left.J^{\nu}\right|_{\Lambda}$. Besides that, we should 
require that the maximal linearly independent subset of the functions (2.7) give a complete set of linearly independent left eigenvectors of the operator $\hat{L}_{j\left[\mathbf{U}, \boldsymbol{\theta}_{0}\right]}^{i}$ with zero eigenvalues among the vectors regularly depending on the parameters $\mathbf{U}$ on the family $\Lambda$.

Coming back to the definition of a complete regular family of $m$-phase solutions of system (2.3) we can see that in the case of a complete regular family $\Lambda$ the number of linearly independent vectors (2.7) on $\Lambda$ is always finite. More precisely, if $N=2 m+s$ is the number of parameters of $m$-phase solutions of (2.3) (excluding the initial phase shifts) then for a complete regular family of $m$-phase solutions we require the presence of exactly $m+s=N-m$ left eigenvectors $\boldsymbol{\kappa}_{[\mathbf{U}]}^{(q)}\left(\boldsymbol{\theta}+\boldsymbol{\theta}_{0}\right)$ with zero eigenvalues, regularly depending on parameters, in accordance with the number of the vectors $\boldsymbol{\Phi}_{\theta^{\alpha}}, \boldsymbol{\Phi}_{n^{l}}$. Thus, according to Definition 1.1, we assume here that the number of linearly independent vectors defined by formula (2.7) is exactly equal to $m+s=N-m$ for a complete regular family $\Lambda$.

We should note that the conditions on the variational derivatives of $J^{\nu}$ formulated above do not contradict to the condition that the values $J^{\nu}(\nu=1, \ldots, N)$ can be chosen as parameters $U^{\nu}$ on the family of $m$-phase solutions. Indeed, the definition of $J^{\nu}(2.6)$ explicitly includes the additional $m$ functions $k^{\alpha}$, which provide the necessary functional independence of the values of $J^{\nu}$ on $\Lambda$. In other words, we can use the Euler-Lagrange expressions for the variational derivatives of $I^{\nu}$ only on subspaces with fixed quasiperiods $\left(k^{1}, \ldots, k^{m}\right)$. The variation of the quasiperiods gives linearly growing variations which do not allow to use the Euler-Lagrange expressions.

Moreover, under the assumptions formulated above, we can show that the condition of the completeness of the variational derivatives (2.7) of the functionals $J^{\nu}$ in the space of regular left eigenvectors of the operator $\hat{L}_{j\left[\mathbf{U}, \boldsymbol{\theta}_{0}\right]}^{i}$ with zero eigenvalues follows in fact from the condition that the values $U^{\nu}=\left.J^{\nu}\right|_{\Lambda}$ can be chosen as the full set of parameters (excluding the initial phase shifts) on the family $\Lambda$.

Let us make the agreement that we will always assume here that the Jacobian of the coordinate transformation

$$
(\mathbf{k}, \boldsymbol{\omega}, \mathbf{n}) \rightarrow\left(U^{1}, \ldots, U^{N}\right)
$$

is different from zero on $\Lambda$ whenever we say that the values $U^{\nu}(\mathbf{k}, \boldsymbol{\omega}, \mathbf{n})$ represent a complete set of parameters on $\Lambda$ (excluding the initial phase shifts).

Under the conditions formulated above let us prove here the following proposition.

Proposition 2.1. Let $\Lambda$ be a complete regular family of $m$-phase solutions of system (2.3). Let the values $\left(U^{1}, \ldots, U^{N}\right)$ of the functionals $\left(J^{1}, \ldots, J^{N}\right)(2.6)$ give a complete set of parameters on $\Lambda$ excluding the initial phase shifts. Then:

1) the set of the vectors

$$
\left\{\boldsymbol{\Phi}_{\omega^{\alpha}}\left(\boldsymbol{\theta}+\boldsymbol{\theta}_{0}, \mathbf{k}, \boldsymbol{\omega}, \mathbf{n}\right), \boldsymbol{\Phi}_{n^{l}}\left(\boldsymbol{\theta}+\boldsymbol{\theta}_{0}, \mathbf{k}, \boldsymbol{\omega}, \mathbf{n}\right), \alpha=1, \ldots, m, l=1, \ldots, s\right\}
$$

is linearly independent on $\Lambda$;

2) the variation derivatives $\zeta_{i[\mathbf{U}]}^{(\nu)}\left(\boldsymbol{\theta}+\boldsymbol{\theta}_{0}\right)$, given by (2.7), generate the full space of the regular left eigenvectors of the operator $\hat{L}_{j\left[\mathbf{U}, \boldsymbol{\theta}_{0}\right]}^{i}$ with zero eigenvalues on the family $\Lambda$.

Proof. Indeed, we require that the rows given by the derivatives

$$
\left(\frac{\partial U^{1}}{\partial \omega^{\alpha}}, \ldots, \frac{\partial U^{N}}{\partial \omega^{\alpha}}\right), \quad\left(\frac{\partial U^{1}}{\partial n^{l}}, \ldots, \frac{\partial U^{N}}{\partial n^{l}}\right)
$$


are linearly independent on $\Lambda$. Using the expressions

$$
\begin{array}{ll}
\frac{\partial U^{\nu}}{\partial \omega^{\alpha}}=\int_{0}^{2 \pi} \cdots \int_{0}^{2 \pi} \zeta_{i[\mathbf{U}]}^{(\nu)}(\boldsymbol{\theta}) \Phi_{\omega^{\alpha}}^{i}(\boldsymbol{\theta}, \mathbf{U}) \frac{\mathrm{d}^{m} \theta}{(2 \pi)^{m}}, & \alpha=1, \ldots, m, \\
\frac{\partial U^{\nu}}{\partial n^{l}}=\int_{0}^{2 \pi} \cdots \int_{0}^{2 \pi} \zeta_{i[\mathbf{U}]}^{(\nu)}(\boldsymbol{\theta}) \Phi_{n^{l}}^{i}(\boldsymbol{\theta}, \mathbf{U}) \frac{\mathrm{d}^{m} \theta}{(2 \pi)^{m}}, & l=1, \ldots, s,
\end{array}
$$

on $\Lambda$, we get that the set $\left\{\boldsymbol{\Phi}_{\omega^{\alpha}}, \boldsymbol{\Phi}_{n^{l}}\right\}$ is linearly independent on $\Lambda$ and the number of linearly independent variation derivatives (2.7) is not less than $m+s$.

We obtain then that the variation derivatives (2.7) generate in this case a space of regular left eigenvectors of the operator $\hat{L}_{j\left[\mathbf{U}, \boldsymbol{\theta}_{0}\right]}^{i}$ with zero eigenvalues of dimension $(m+s)$.

As a remark, let us note here some general fact associated with multiphase solutions of partial differential equations. As is well known, the presence of families of multiphase quasiperiodic solutions, as a rule, is connected with integrability of system (1.1) by the inverse scattering methods.

The first $m$-phase solutions for the KdV equation given by the Novikov potentials were introduced exactly as the functional families represented by the extremals of linear combinations of some set of higher integrals of the system, i.e. the families where the variational derivatives of integrals of the set become linearly dependent.

If we have a natural hierarchy of the first integrals and commuting flows of an integrable system, some of the first integrals of the hierarchy $\left(I^{1}, \ldots, I^{q}\right)$ are usually used for the construction of $m$-phase solutions. So, the functions given by the conditions

$$
c_{1} \delta I^{1}+\cdots+c_{q} \delta I^{q}=0
$$

for all possible $\left(c_{1}, \ldots, c_{q}\right)$ form a complete family of $m$-phase solutions of the integrable system [41].

Thus, for Novikov potentials we have $q=m+2$ while the dimensions of the families of $m$-phase solutions for $\mathrm{KdV}$ (excluding initial phase shifts) are equal to $2 m+1$. The first $2 m+1$ of integrals of $\operatorname{KdV}\left(I^{1}, \ldots, I^{2 m+1}\right)$ can be used for the construction of parameters $\left(U^{1}, \ldots, U^{2 m+1}\right)$ on the families of $m$-phase solutions of $\mathrm{KdV}$. The number of linearly independent variational derivatives of these functionals on the family of $m$-phase solutions is exactly equal to $m+1$. It's not difficult to show also that the variational derivatives of all the higher integrals of the KdV hierarchy are given by linear combinations of the variational derivatives of the set $\left(I^{1}, \ldots, I^{q}\right)$ on the families of $m$-phase solutions.

The construction proposed in [41] in fact is used without substantial changes for many systems that are integrable by the inverse scattering methods, and represents the basic scheme for constructing of $m$-phase solutions of integrable systems. This circumstance gives therefore a convenient method of checking the above relations for most specific examples.

Let us prove here the following lemma, which we will need in further considerations.

Lemma 2.1. Let the values $U^{\nu}$ of the functionals $J^{\nu}$ on a complete regular family of $m$-phase solutions $\Lambda$ be functionally independent and give a complete set of parameters (excluding initial phase shifts) on $\Lambda$, such that we have $k^{\alpha}=k^{\alpha}\left(U^{1}, \ldots, U^{N}\right)$. Then the functionals $k^{\alpha}\left(J^{1}, \ldots, J^{N}\right)$ have zero variational derivatives on $\Lambda$.

Proof. As we have seen, the conditions of the lemma imply the existence of $m$ independent relations

$$
\left.\sum_{\nu=1}^{N} \lambda_{\nu}^{\alpha}(\mathbf{U}) \frac{\delta J^{\nu}}{\delta \varphi^{i}(\boldsymbol{\theta})}\right|_{\boldsymbol{\varphi}(\boldsymbol{\theta})=\boldsymbol{\Phi}\left(\boldsymbol{\theta}+\boldsymbol{\theta}_{0}, \mathbf{U}\right)} \equiv 0, \quad \alpha=1, \ldots, m,
$$

on $\Lambda$. 
For the corresponding coordinates $U^{\nu}$ on $\Lambda$ this implies the relations

$$
\sum_{\nu=1}^{N} \lambda_{\nu}^{\alpha}(\mathbf{U}) \mathrm{d} U^{\nu}=\sum_{\beta=1}^{m} \mu_{\beta}^{(\alpha)}(\mathbf{U}) \mathrm{d} k^{\beta}(\mathbf{U})
$$

for some matrix $\mu_{\beta}^{(\alpha)}(\mathbf{U})$.

Since $U^{\nu}$ provide coordinates on $\Lambda$ the matrix $\mu_{\beta}^{(\alpha)}(\mathbf{U})$ has the full rank, and therefore invertible. We can then write

$$
\mathrm{d} k^{\beta}=\sum_{\alpha=1}^{m}\left(\hat{\mu}^{-1}\right)_{(\alpha)}^{\beta}(\mathbf{U}) \sum_{\nu=1}^{N} \lambda_{\nu}^{(\alpha)}(\mathbf{U}) \mathrm{d} U^{\nu} .
$$

The assertion of the lemma follows then from (2.9).

The regular Whitham system in the described approach can be written as

$$
\left\langle P^{\nu}\right\rangle_{T}=\left\langle Q^{\nu}\right\rangle_{X}, \quad \nu=1, \ldots, N
$$

where $\langle\ldots\rangle$ denotes the averaging operation on $\Lambda$ defined by the formula

$$
\left\langle f\left(\boldsymbol{\varphi}, \boldsymbol{\varphi}_{x}, \ldots\right)\right\rangle \equiv \int_{0}^{2 \pi} \cdots \int_{0}^{2 \pi} f\left(\boldsymbol{\Phi}, k^{\beta} \boldsymbol{\Phi}_{\theta^{\beta}}, \ldots\right) \frac{\mathrm{d}^{m} \theta}{(2 \pi)^{m}}
$$

Let us prove here the following lemma about the connection between the systems (2.10) and (1.11).

Lemma 2.2. Let the values $U^{\nu}$ of the functionals $J^{\nu}$ on a complete regular family of $m$-phase solutions $\Lambda$ be functionally independent and give a complete set of parameters on $\Lambda$ excluding the initial phase shifts. Then the system (2.10) is equivalent to (1.11).

Proof. Let us introduce the functions

$$
\Pi_{i(l)}^{\nu}\left(\boldsymbol{\varphi}, \boldsymbol{\varphi}_{x}, \ldots\right) \equiv \frac{\partial P^{\nu}\left(\boldsymbol{\varphi}, \boldsymbol{\varphi}_{x}, \ldots\right)}{\partial \varphi_{l x}^{i}}
$$

for $l \geq 0$.

Using the expression for the evolution of the densities $P^{\nu}\left(\boldsymbol{\varphi}, \epsilon \boldsymbol{\varphi}_{X}, \ldots\right)$ we can write the following identities

$$
P_{t}^{\nu}\left(\boldsymbol{\varphi}, \epsilon \boldsymbol{\varphi}_{X}, \ldots\right)=\sum_{l \geq 0} \epsilon^{l} \Pi_{i(l)}^{\nu}\left(\boldsymbol{\varphi}, \epsilon \boldsymbol{\varphi}_{X}, \ldots\right)\left(F^{i}\left(\boldsymbol{\varphi}, \epsilon \boldsymbol{\varphi}_{X}, \ldots\right)\right)_{l X} \equiv \epsilon Q_{X}^{\nu}\left(\boldsymbol{\varphi}, \epsilon \boldsymbol{\varphi}_{X}, \ldots\right) .
$$

To calculate the values $\epsilon\left\langle Q^{\nu}\right\rangle_{X}$ let us put now

$$
\varphi^{i}(\boldsymbol{\theta}, X)=\Phi^{i}\left(\frac{\mathbf{S}(X)}{\epsilon}+\boldsymbol{\theta}, \mathbf{U}(X)\right),
$$

where $S_{X}^{\alpha}=k^{\alpha}(\mathbf{U}(X))$.

The operator $\epsilon \partial / \partial X$ acting on the functions (2.12) can be naturally represented as a sum of $k^{\alpha} \partial / \partial \theta^{\alpha}$ and the terms proportional to $\epsilon$. So, any expression $f\left(\boldsymbol{\varphi}, \epsilon \boldsymbol{\varphi}_{X}, \ldots\right)$ on the submanifold (2.12) can be naturally represented in the form

$$
f\left(\boldsymbol{\varphi}, \epsilon \boldsymbol{\varphi}_{X}, \ldots\right)=\sum_{l \geq 0} \epsilon^{l} f_{[l]}[\mathbf{\Phi}, \mathbf{U}],
$$


where $f_{[l]}[\mathbf{\Phi}, \mathbf{U}]$ are smooth functions of $\left(\boldsymbol{\Phi}, \boldsymbol{\Phi}_{\theta^{\alpha}}, \boldsymbol{\Phi}_{U^{\nu}}, \ldots\right)$ and $\left(\mathbf{U}, \mathbf{U}_{X}, \mathbf{U}_{X X}, \ldots\right)$, polynomial in the derivatives $\left(\mathbf{U}_{X}, \mathbf{U}_{X X}, \ldots\right)$, and having degree $l$ in terms of the total number of derivations of $\mathbf{U}$ w.r.t. $X$. Note also that the functions $\boldsymbol{\Phi}$ appear in $f_{[l]}$ with the phase shift $\mathbf{S}(X) / \epsilon$ according to (2.12). The common phase shift is not important for the integration with respect to $\boldsymbol{\theta}$, so let us assume below that the phase shift $\mathbf{S}(X) / \epsilon$ is omitted after taking all the differentiations with respect to $X$.

According to (2.11) and (2.4) we can write

$$
\begin{aligned}
& \epsilon\left\langle Q^{\nu}\right\rangle_{X}=\epsilon \int_{0}^{2 \pi} \cdots \int_{0}^{2 \pi} Q_{X[1]}^{\nu} \frac{\mathrm{d}^{m} \theta}{(2 \pi)^{m}} \\
& =\epsilon \int_{0}^{2 \pi} \cdots \int_{0}^{2 \pi} \sum_{l \geq 0}\left(\Pi_{i(l)[0]}^{\nu} F_{l X[1]}^{i}+\Pi_{i(l)[1]}^{\nu} F_{l X[0]}^{i}\right) \frac{\mathrm{d}^{m} \theta}{(2 \pi)^{m}} \\
& =\epsilon \int_{0}^{2 \pi} \cdots \int_{0}^{2 \pi} \sum_{l \geq 0}\left(\Pi_{i(l)[0]}^{\nu} k^{\gamma_{1}} \cdots k^{\gamma_{l}} F_{[1] \theta^{\gamma_{1}} \ldots \theta^{\gamma_{l}}}^{i}\right. \\
& +\Pi_{i(l)[0]}^{\nu} l k^{\gamma_{1}} \cdots k^{\gamma_{l-1}}\left(\omega^{\beta} \Phi_{\theta^{\beta} \theta^{\gamma_{1} \ldots \theta^{\gamma_{l-1}}}}^{i}\right)_{X[1]} \\
& +\Pi_{i(l)[0]}^{\nu} \frac{l(l-1)}{2} k_{X}^{\gamma_{1}} k^{\gamma_{2}} \cdots k^{\gamma_{l-1}} \omega^{\beta} \Phi_{\theta^{\beta} \theta^{\gamma_{1} \ldots \theta^{\gamma} l-1}}^{i} \\
& \left.+\Pi_{i(l)[1]}^{\nu} k^{\gamma_{1}} \ldots k^{\gamma_{l}} \omega^{\beta} \Phi_{\theta^{\beta} \theta^{\gamma_{1} \ldots \theta^{\gamma_{l}}}}^{i}\right) \frac{\mathrm{d}^{m} \theta}{(2 \pi)^{m}} .
\end{aligned}
$$

It is not difficult to see also that for arbitrary dependence of parameters $\mathbf{U}$ of $T$, the derivative of the average $\left\langle P^{\nu}\right\rangle$ w.r.t. $T$ can be written as

$$
\left\langle P^{\nu}\right\rangle_{T}=\int_{0}^{2 \pi} \cdots \int_{0}^{2 \pi} \sum_{l \geq 0} \Pi_{i(l)[0]}^{\nu}\left(k^{\gamma_{1}} \cdots k^{\gamma_{l}} \Phi_{\theta^{\gamma_{1} \ldots \theta^{\gamma_{l}}}}^{i}\right)_{T} \frac{\mathrm{d}^{m} \theta}{(2 \pi)^{m}} .
$$

Now, we can write the relations $\left\langle P^{\nu}\right\rangle_{T}=\left\langle Q^{\nu}\right\rangle_{X}$ as

$$
\begin{aligned}
& \int_{0}^{2 \pi} \cdots \int_{0}^{2 \pi} \sum_{l \geq 0}\left(\Pi_{i(l)[0]}^{\nu} k^{\gamma_{1}} \cdots k^{\gamma_{l}} \Phi_{\theta^{\gamma_{1} \ldots \theta^{\gamma_{l} T}}}^{i}+\Pi_{i(l)[0]}^{\nu} l k^{\gamma_{1}} \cdots k^{\gamma_{l-1}} k_{T}^{\gamma_{l}} \Phi_{\theta^{\gamma_{1} \ldots \theta^{\gamma_{l}}}}^{i}\right) \frac{\mathrm{d}^{m} \theta}{(2 \pi)^{m}} \\
& =\int_{0}^{2 \pi} \cdots \int_{0}^{2 \pi} \sum_{l \geq 0}\left(\Pi_{i(l)[0]}^{\nu} k^{\gamma_{1}} \cdots k^{\gamma_{l}} F_{[1] \theta^{\gamma_{1} \ldots \theta^{\gamma l}}}^{i}\right.
\end{aligned}
$$

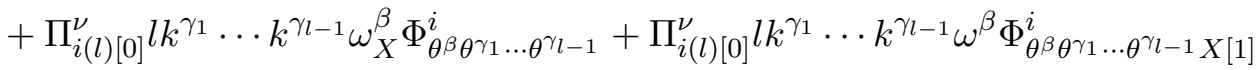

$$
\begin{aligned}
& \left.+\Pi_{i(l)[0]}^{\nu} \frac{l(l-1)}{2} k_{X}^{\gamma_{1}} k^{\gamma_{2}} \cdots k^{\gamma_{l-1}} \omega^{\beta} \Phi_{\theta^{\beta} \theta^{\gamma_{1} \ldots \theta^{\gamma_{l-1}}}}^{i}+\Pi_{i(l)[1]}^{\nu} k^{\gamma_{1}} \cdots k^{\gamma_{l}} \omega^{\beta} \Phi_{\theta^{\beta} \theta^{\gamma_{1} \ldots \theta^{\gamma_{l}}}}^{i}\right) \frac{\mathrm{d}^{m} \theta}{(2 \pi)^{m}} .
\end{aligned}
$$

The last three terms in the right-hand part represent the integral of the value

$$
\sum_{l \geq 0} \omega^{\beta}\left(\Pi_{i(l)[0]}^{\nu} \Phi_{\theta^{\beta}, l X[1]}^{i}+\Pi_{i(l)[1]}^{\nu} \Phi_{\theta^{\beta}, l X[0]}^{i}\right)=\omega^{\beta} \partial P_{[1]}^{\nu} / \partial \theta^{\beta}
$$

and are equal to zero. The remaining terms after integration by parts can be written in the form

$$
\begin{aligned}
\int_{0}^{2 \pi} \cdots \int_{0}^{2 \pi}( & \zeta_{i[\mathbf{U}(X)]}^{(\nu)}(\boldsymbol{\theta})\left[\Phi_{T}^{i}(\boldsymbol{\theta}, \mathbf{U}(X))-F_{[1]}^{i}(\boldsymbol{\theta}, X)\right] \\
& \left.+\left(k_{T}^{\beta}-\omega_{X}^{\beta}\right) \sum_{l \geq 0} \Pi_{i(l)[0]}^{\nu} l k^{\gamma_{1}} \cdots k^{\gamma_{l-1}} \Phi_{\theta^{\beta} \theta^{\gamma_{1} \ldots \theta^{\gamma} l-1}}^{i}\right) \frac{\mathrm{d}^{m} \theta}{(2 \pi)^{m}}=0
\end{aligned}
$$

where the values $\zeta_{i[\mathbf{U}(X)]}^{(\nu)}(\boldsymbol{\theta})$ are given by $(2.7)$. 
Consider the convolution (in $\nu$ ) of the above expression with the values $\partial k^{\alpha} / \partial U^{\nu}$. The expressions

$$
\frac{\partial k^{\alpha}}{\partial U^{\nu}}(\mathbf{U}(X)) \zeta_{i[\mathbf{U}(X)]}^{(\nu)}(\boldsymbol{\theta})
$$

are identically equal to zero according to Lemma 2.1 .

From the other hand we have

$$
\begin{gathered}
\frac{\partial k^{\alpha}}{\partial U^{\nu}} \int_{0}^{2 \pi} \cdots \int_{0}^{2 \pi} \sum_{l \geq 1} l k^{\beta_{1}} \ldots k^{\beta_{l-1}} \Phi_{\theta^{\beta} \theta^{\beta_{1} \ldots \theta^{\beta_{l-1}}}} \Pi_{i(l)}^{\nu}\left(\boldsymbol{\Phi}, k^{\gamma} \boldsymbol{\Phi}_{\theta^{\gamma}}, \ldots\right) \frac{\mathrm{d}^{m} \theta}{(2 \pi)^{m}} \\
\quad=\left.\left(\frac{\partial k^{\alpha}}{\partial U^{\nu}} \frac{\partial}{\partial k^{\beta}} J^{\nu}[\boldsymbol{\varphi}, \mathbf{k}]\right)\right|_{\boldsymbol{\varphi}(\boldsymbol{\theta})=\boldsymbol{\Phi}(\boldsymbol{\theta}, \mathbf{U})}=\delta_{\beta}^{\alpha},
\end{gathered}
$$

since the variations of the functions $\boldsymbol{\Phi}$ are insignificant for the values of $k^{\alpha}$ according to Lemma 2.1.

We get then that conditions (2.10) imply the relations $k_{T}^{\alpha}=\omega_{X}^{\alpha}$, which are the first part of system (1.11).

Now the conditions

$$
\int_{0}^{2 \pi} \cdots \int_{0}^{2 \pi} \zeta_{i[\mathbf{U}(X)]}^{(\nu)}(\boldsymbol{\theta})\left[\Phi_{T}^{i}(\boldsymbol{\theta}, \mathbf{U}(X))-F_{[1]}^{i}(\boldsymbol{\theta}, X)\right] \frac{\mathrm{d}^{m} \theta}{(2 \pi)^{m}}=0
$$

express the conditions of orthogonality of the vectors (2.7) to the function $-\mathbf{\Phi}_{T}+\mathbf{F}_{[1]}$, which coincides exactly with the right-hand part of the equation (1.7) in our case. Since the linear span of the vectors (2.7) coincides with the linear span of the complete set of the regular left eigenvectors of the operator $\hat{L}_{j\left[\mathbf{U}, \boldsymbol{\theta}_{0}\right]}^{i}(X, T)$ with zero eigenvalues, we get that system $(2.10)$ is equivalent to system (1.11).

Let us note here that it follows from Lemma 2.2 that systems (2.10), obtained from different sets of conservation laws are equivalent to each other. In other words, if system (2.3) has additional conservation laws then their averaging gives relations following from system (2.10).

Let us note also that the justification questions discussed above, as a rule, can be considered in a simpler way under additional assumptions about the next corrections to the main approximation (1.5) (see, e.g. [7]). We note again that here we don't make any additional assumptions of this kind and consider the regular Whitham system as an independent object that is associated only with description of the main approximation (1.5). As we have said, we will follow this approach everywhere in the paper.

The Hamiltonian properties of systems (2.10) and more general systems (1.8) play very important role in their consideration. The general theory of systems (1.8), which are Hamiltonian with respect to local Poisson brackets of hydrodynamic type (Dubrovin-Novikov brackets) was constructed by B.A. Dubrovin and S.P. Novikov. Let us give here a brief description of the Dubrovin-Novikov Hamiltonian structures and of the properties of the corresponding systems (1.8).

The Dubrovin-Novikov bracket on the space of fields $\left(U^{1}(X), \ldots, U^{N}(X)\right)$ has the form

$$
\left\{U^{\nu}(X), U^{\mu}(Y)\right\}=g^{\nu \mu}(\mathbf{U}) \delta^{\prime}(X-Y)+b_{\gamma}^{\nu \mu}(\mathbf{U}) U_{X}^{\gamma} \delta(X-Y), \quad \nu, \mu=1, \ldots, N .
$$

The Hamiltonian operator corresponding to (2.14) can be written in the form

$$
\hat{J}^{\nu \mu}=g^{\nu \mu}(\mathbf{U}) \frac{\mathrm{d}}{\mathrm{d} X}+b_{\gamma}^{\nu \mu}(\mathbf{U}) U_{X}^{\gamma} .
$$

As was shown by B.A. Dubrovin and S.P. Novikov [14-16], expression (2.14) with nondegenerate tensor $g^{\nu \mu}(\mathbf{U})$ defines a Poisson bracket on the space of fields $\mathbf{U}(X)$ if and only if: 
1) tensor $g^{\nu \mu}(\mathbf{U})$ gives a symmetric flat pseudo-Riemannian metric with upper indexes on the space of parameters $\left(U^{1}, \ldots, U^{N}\right)$;

2) the values

$$
\Gamma_{\mu \gamma}^{\nu}=-g_{\mu \lambda} b_{\gamma}^{\lambda \nu}
$$

where $g^{\nu \lambda}(\mathbf{U}) g_{\lambda \mu}(\mathbf{U})=\delta_{\mu}^{\nu}$, represent the Christoffel symbols for the corresponding metric $g_{\nu \mu}(\mathbf{U})$.

As follows from the statements above, every Dubrovin-Novikov bracket with non-degenerate tensor $g^{\nu \mu}(\mathbf{U})$ can be written in the canonical form [14-16]

$$
\left\{n^{\nu}(X), n^{\mu}(Y)\right\}=\epsilon^{\nu} \delta^{\nu \mu} \delta^{\prime}(X-Y), \quad \epsilon^{\nu}= \pm 1,
$$

after the transition to the flat coordinates $n^{\nu}=n^{\nu}(\mathbf{U})$ for the metric $g_{\nu \mu}(\mathbf{U})$.

The functionals

$$
N^{\nu}=\int_{-\infty}^{+\infty} n^{\nu}(X) \mathrm{d} X
$$

represent the annihilators of the Dubrovin-Novikov bracket while the functional

$$
P=\int_{-\infty}^{+\infty} \frac{1}{2} \sum_{\nu=1}^{N} \epsilon^{\nu}\left(n^{\nu}\right)^{2}(X) \mathrm{d} X
$$

represents the momentum functional for the bracket (2.14).

The Hamiltonian functions in the theory of brackets (2.14) are represented by the functionals of hydrodynamic type, i.e.

$$
H=\int_{-\infty}^{+\infty} h(\mathbf{U}) \mathrm{d} X
$$

The bracket (2.14) has also two other important forms on the space of $\mathbf{U}(X)$. One of them is the "Liouville" form [14-16] having the form

$$
\left\{U^{\nu}(X), U^{\mu}(Y)\right\}=\left(\gamma^{\nu \mu}(\mathbf{U})+\gamma^{\mu \nu}(\mathbf{U})\right) \delta^{\prime}(X-Y)+\frac{\partial \gamma^{\nu \mu}}{\partial U^{\lambda}} U_{X}^{\lambda} \delta(X-Y)
$$

for some functions $\gamma^{\nu \mu}(\mathbf{U})$.

The "Liouville" form of the Dubrovin-Novikov bracket is called also the physical form and corresponds to the case when the integrals of coordinates $U^{\nu}$

$$
I^{\nu}=\int_{-\infty}^{+\infty} U^{\nu}(X) \mathrm{d} X
$$

commute with each other.

Another important form of the Dubrovin-Novikov bracket is the diagonal form. It corresponds to the case when the coordinates $U^{\nu}$ represent the diagonal coordinates for the metric $g_{\nu \mu}(\mathbf{U})$ and the tensor $g^{\nu \mu}(\mathbf{U})$ in $(2.14)$ has a diagonal form. This form of the DubrovinNovikov bracket is closely connected with the integration theory of systems of hydrodynamic type

$$
U_{T}^{\nu}=V_{\mu}^{\nu}(\mathbf{U}) U_{X}^{\mu}
$$


which can be written in the diagonal form

$$
U_{T}^{\nu}=V^{\nu}(\mathbf{U}) U_{X}^{\nu}
$$

(no summation) and are Hamiltonian with respect to bracket (2.14).

It was conjectured by S.P. Novikov that all the systems of hydrodynamic type having the form (2.15) and Hamiltonian with respect to any bracket (2.14) are integrable. This conjecture was proved by S.P. Tsarev in [46] where the method (the "generalized hodograph method") of integration of these systems was suggested. However, the method of Tsarev proved to be applicable to a wider class of diagonalizable systems of hydrodynamic type which was called by Tsarev "semi-Hamiltonian". As it turned out later in the class of "semi-Hamiltonian systems" fall also the systems Hamiltonian with respect to generalizations of the Dubrovin-Novikov bracket - the weakly nonlocal Mokhov-Ferapontov bracket [38] and the Ferapontov bracket [18,21]. Various aspects of the weakly nonlocal brackets of hydrodynamic type are discussed in [18-21,36,38,43]. Let us note also that the generalization of the Dubrovin-Novikov procedure for weakly nonlocal case was proposed in [34].

Let us describe now the procedure for constructing the Dubrovin-Novikov bracket for the Whitham system in the case when the original system (2.3) is Hamiltonian with respect to a local field-theoretic bracket

$$
\left\{\varphi^{i}(x), \varphi^{j}(y)\right\}=\sum_{k \geq 0} B_{(k)}^{i j}\left(\boldsymbol{\varphi}, \boldsymbol{\varphi}_{x}, \ldots\right) \delta^{(k)}(x-y)
$$

with the local Hamiltonian of the form

$$
H=\int P_{H}\left(\varphi, \varphi_{x}, \ldots\right) \mathrm{d} x
$$

which was suggested by B.A. Dubrovin and S.P. Novikov [14-16].

Method of B.A. Dubrovin and S.P. Novikov is based on the existence of $N$ (equal to the number of parameters $U^{\nu}$ of the family of $m$-phase solutions of (2.3)) local integrals

$$
I^{\nu}=\int P^{\nu}\left(\varphi, \varphi_{x}, \ldots\right) \mathrm{d} x
$$

which commute with the Hamiltonian (2.17) and with each other

$$
\left\{I^{\nu}, H\right\}=0, \quad\left\{I^{\nu}, I^{\mu}\right\}=0
$$

and can be described as follows.

We calculate the pairwise Poisson brackets of the densities $P^{\nu}$ having the form

$$
\left\{P^{\nu}(x), P^{\mu}(y)\right\}=\sum_{k \geq 0} A_{k}^{\nu \mu}\left(\boldsymbol{\varphi}, \boldsymbol{\varphi}_{x}, \ldots\right) \delta^{(k)}(x-y)
$$

where

$$
A_{0}^{\nu \mu}\left(\boldsymbol{\varphi}, \boldsymbol{\varphi}_{x}, \ldots\right) \equiv \partial_{x} Q^{\nu \mu}\left(\boldsymbol{\varphi}, \boldsymbol{\varphi}_{x}, \ldots\right)
$$

according to (2.19).

The corresponding Dubrovin-Novikov bracket on the space of functions $\mathbf{U}(X)$ has the form

$$
\left\{U^{\nu}(X), U^{\mu}(Y)\right\}=\left\langle A_{1}^{\nu \mu}\right\rangle(\mathbf{U}) \delta^{\prime}(X-Y)+\frac{\partial\left\langle Q^{\nu \mu}\right\rangle}{\partial U^{\gamma}} U_{X}^{\gamma} \delta(X-Y) .
$$


Let us remind that we assume that the parameters $U^{\nu}$ coincide with the values of the functionals $I^{\nu}$ defined on the corresponding quasiperiodic solutions of the family $\Lambda$

$$
U^{\nu}=\left\langle P^{\nu}(x)\right\rangle .
$$

The Whitham system (2.10) is Hamiltonian with respect to the Dubrovin-Novikov bracket (2.21) with the Hamiltonian

$$
H^{a v}=\int_{-\infty}^{+\infty}\left\langle P_{H}\right\rangle(\mathbf{U}(X)) \mathrm{d} X
$$

The proof of the Jacobi identity for the bracket (2.21) was suggested in [32] under certain assumptions about the family of $m$-phase solutions of (2.3). Besides that, it was shown in [37] that the Dubrovin-Novikov procedure is compatible with the procedure of averaging of local Lagrangian functions when carrying out of both the procedures is possible.

Let us note also that in [44] all the local brackets (2.14) for the Whitham equations for KdV, NLS, and SG equations were found. Besides that, in [2] the hierarchies of the weakly nonlocal Hamiltonian structures for the Whitham systems for KdV were represented.

In this paper we give the most detailed discussion and justification of the Dubrovin-Novikov procedure separately for the single-phase and the multiphase cases. In the multiphase case we will show also that the justification of the Dubrovin-Novikov procedure can be done under weaker assumptions than in previous reviews. In particular, we will show that the justification of the procedure is in fact insensitive to the appearance of "resonances" which can arise in the multi-phase case, which is the basis for its widespread use in the multiphase situation. In all cases we will consider the regular Whitham system defined above without any assumptions about the form of higher corrections to the main approximation (1.5) of a slowly modulated solution.

In this chapter we consider general constructions connected with the Dubrovin-Novikov procedure and prove some technical lemmas needed for its justification. At the end of the chapter, we give a detailed justification of the procedure in the single-phase case. In the next chapter we will consider multiphase case, where the justification of the Dubrovin-Novikov procedure will have a more complicated form. In conclusion, we consider a simple but typical example of the justification of the Dubrovin-Novikov procedure using the Gardner-Zakharov-Faddeev bracket for $\mathrm{KdV}$.

As we said above, we will now consider the general structures connected with the construction of the Hamiltonian structure for the regular Whitham system.

Let us note that according to (2.19) the flows

$$
\varphi_{t^{\nu}}^{i}=S^{i \nu}\left(\boldsymbol{\varphi}, \varphi_{x}, \ldots\right)=\left\{\varphi^{i}(x), I^{\nu}\right\}
$$

generated by the functionals $I^{\nu}$ according to the bracket (2.16) commute with the initial flow (2.3). The flows (2.23) leave invariant the full families of $m$-phase solutions of (2.3) as well as the values $U^{\nu}=I^{\nu}$ of the functionals $I^{\nu}$ on them. For a complete regular family of $m$-phase solutions with independent parameters $\left(k^{1}, \ldots, k^{m}\right)$ it's not difficult to show that the flows (2.23) generate linear (in time) shifts of the phases $\theta_{0}^{\alpha}$ with some constant frequencies $\omega^{\alpha \nu}(\mathbf{U})$, such that

$$
S^{i \nu}\left(\boldsymbol{\Phi}, k^{\beta} \boldsymbol{\Phi}_{\theta^{\beta}}, \ldots\right)=\omega^{\alpha \nu}(\mathbf{U}) \Phi_{\theta^{\alpha}}^{i}(\boldsymbol{\theta}, \mathbf{U}) .
$$

According to Lemma 2.1 we have also that the functionals $k^{\alpha}(\mathbf{I})$ should generate the zero flows on the corresponding family of $m$-phase solutions of (2.3). For $U^{\nu}$ coinciding with the 
values of $I^{\nu}$ on $\Lambda$ we get then the relations

$$
\frac{\partial k^{\alpha}(\mathbf{U})}{\partial U^{\nu}} \omega^{\beta \nu}(\mathbf{U}) \equiv 0, \quad \alpha, \beta=1, \ldots, m
$$

Let us give here the definition of a regular Hamiltonian family of $m$-phase solutions of system (2.3) and of a complete Hamiltonian set of the functionals (2.18).

Definition 2.1. We call family $\Lambda$ of $m$-phase solutions of system (2.3) a regular Hamiltonian family if:

1) it represents a complete regular family of $m$-phase solutions of $(2.3)$ in the sense of Definition 1.1;

2) the corresponding bracket (2.16) has on $\Lambda$ constant number of annihilators $N^{1}, \ldots, N^{s}$ with linearly independent variational derivatives $\delta N^{l} / \delta \varphi^{i}(x)$ which coincides with the number of independent annihilators in the neighborhood of $\Lambda$.

Let us say that according to the generalized Darboux theorem we can identify the number of the variational derivatives $\delta N^{l} / \delta \varphi^{i}(x)$ on $\Lambda$ with the number of linearly independent quasiperiodic solutions $v_{i}^{(l)}(x)$ of the equation

$$
\left.\sum_{k \geq 0} B_{(k)}^{i j}\left(\boldsymbol{\varphi}, \boldsymbol{\varphi}_{x}, \ldots\right)\right|_{\Lambda} v_{j, k x}^{(l)}=0
$$

where $v_{i}^{(l)}(x)$ have the same quasiperiods as the corresponding functions $\varphi(x)$ on $\Lambda$.

Definition 2.2. We call a set $\left(I^{1}, \ldots, I^{N}\right)$ of commuting functionals (2.18) a complete Hamiltonian set on a regular Hamiltonian family $\Lambda$ of $m$-phase solutions of system (2.3) if:

1) the restriction of the functionals $\left(I^{1}, \ldots, I^{N}\right)$ on the quasiperiodic solutions of the family $\Lambda$ gives a complete set of parameters $\left(U^{1}, \ldots, U^{N}\right)$ on this family;

2 ) the Hamiltonian flows generated by $\left(I^{1}, \ldots, I^{N}\right)$ generate on $\Lambda$ linear phase shifts of $\boldsymbol{\theta}_{0}$ with frequencies $\boldsymbol{\omega}^{\nu}(\mathbf{U})$, such that

$$
\operatorname{rank}\left\|\omega^{\alpha \nu}(\mathbf{U})\right\|=m
$$

3) the linear space generated by the variational derivatives $\delta I^{\nu} / \delta \varphi^{i}(x)$ on $\Lambda$ contains the variational derivatives of all the annihilators $N^{q}$ of the bracket (2.16), such that

$$
\left.\frac{\delta N^{l}}{\delta \varphi^{i}(x)}\right|_{\Lambda}=\left.\sum_{\nu} \gamma_{\nu}^{l}(\mathbf{U}) \frac{\delta I^{\nu}}{\delta \varphi^{i}(x)}\right|_{\Lambda}
$$

for some smooth functions $\gamma_{\nu}^{l}(\mathbf{U})$ on the family $\Lambda$.

Let us note that it follows from Definition 2.2 that if a complete Hamiltonian set of integrals $\left(I^{1}, \ldots, I^{N}\right)$ exists for a regular Hamiltonian family $\Lambda$ then the number of the additional parameters $\left(n^{1}, \ldots, n^{s}\right)$ discussed above is equal to the number of annihilators of the bracket (2.16). Indeed, according to Definitions 2.1 and 2.2, the number of the functionals $I^{\nu}$ having linearly independent variational derivatives on $\Lambda$ exactly equals to $m+s$, where $s$ is the number of annihilators of the bracket (2.16). The total number of independent parameters $U^{\nu}$ on $\Lambda$ is then equal to $2 m+s$ due to the wave vectors $k^{\alpha}, \alpha=1, \ldots, m$, which implies the above assertion.

As follows from the condition (2) of Definition 2.2 and from the invariance of the functionals $N^{l}$ and $I^{\nu}$ with respect to the flows $(2.23)$, the values $\gamma_{\nu}^{l}(\mathbf{U})$ can always be chosen independent on the initial phase shifts on the family $\Lambda$. The values $\delta I^{\nu} /\left.\delta \varphi^{i}(x)\right|_{\Lambda}$ are linearly dependent 
on $\Lambda$, so it's natural to choose a complete linearly independent subsystem. Remembering that the variational derivatives of $J^{\nu}(2.7)$ are linear combinations of the regular left eigenvectors $\kappa_{i[\mathbf{U}]}^{(q)}\left(\boldsymbol{\theta}+\boldsymbol{\theta}_{0}\right)$, we can write

$$
\left.\frac{\delta N^{l}}{\delta \varphi^{i}(x)}\right|_{\varphi(x)=\boldsymbol{\Phi}\left(\mathbf{k}(\mathbf{U}) x+\boldsymbol{\theta}_{0}, \mathbf{U}\right)}=\sum_{q} n_{q}^{l}(\mathbf{U}) \kappa_{i[\mathbf{U}]}^{(q)}\left(\mathbf{k}(\mathbf{U}) x+\boldsymbol{\theta}_{0}\right)
$$

for some smooth functions $n_{q}^{l}(\mathbf{U})$. The functions $n_{q}^{l}(\mathbf{U})$ are then uniquely determined on $\Lambda$ and we have $\operatorname{rank}\left\|n_{q}^{l}(\mathbf{U})\right\|=s$ by Definition 2.1 .

We will need now the construction of the Dirac restriction of a Poisson bracket on a submanifold. We describe here briefly this construction. Using the terminology of finite-dimensional spaces we can say that the Dirac restriction of a Poisson bracket on a submanifold $\mathcal{N}^{k} \subset \mathcal{M}^{n}$ is associated with a special choice of coordinates in the neighborhood of the submanifold $\mathcal{N}^{k}$. The coordinates in the neighborhood of the submanifold $\mathcal{N}^{k}$ are divided into the "coordinates on the submanifold" $\left(U^{1}, \ldots, U^{k}\right)$ and the constraints $\left(g^{1}, \ldots, g^{n-k}\right)$ which define the submanifold $\mathcal{N}^{k}$. It is assumed that the submanifold $\mathcal{N}^{k}$ is defined by the conditions

$$
g^{i}(\mathbf{x})=0, \quad i=1, \ldots, n-k,
$$

while the functions $U^{1}(\mathbf{x}), \ldots, U^{k}(\mathbf{x})$ on $\mathcal{M}^{n}$ play the role of a coordinate system on $\mathcal{N}^{k}$ after the restriction to this submanifold.

If the Hamiltonian flows generated by the functions $U^{j}(\mathbf{x})$ leave the submanifold $\mathcal{N}^{k}$ invariant, i.e. we have

$$
\left\{U^{j}(\mathbf{x}), g^{i}(\mathbf{x})\right\}=0 \quad \text { for } \quad \mathbf{g}(\mathbf{x})=0,
$$

then the pairwise Poisson brackets of the functions $U^{j}(\mathbf{x})$ define the Poisson tensor in the coordinates $\left(U^{1}, \ldots, U^{k}\right)$ after restriction on $\mathcal{N}^{k}$ which is called the Dirac restriction of the Poisson bracket $\{\cdot, \cdot\}$ on the submanifold $\mathcal{N}^{k} \subset \mathcal{M}^{n}$.

In general, according to the procedure of Dirac, if we have some constraints $g^{i}(\mathbf{x})$ which define the submanifold $\mathcal{N}^{k}$ and some functions $U^{j}(\mathbf{x})$ which give a coordinate system on $\mathcal{N}^{k}$ we must find $k$ linear combinations $\beta_{s}^{j}(\mathbf{U}) g^{s}(\mathbf{x})$ at every point of $\mathcal{N}^{k}$, such that for the functions

$$
\tilde{U}^{j}(\mathbf{x})=U^{j}(\mathbf{x})+\beta_{s}^{j}(\mathbf{U}) g^{s}(\mathbf{x}), \quad j=1, \ldots, k,
$$

we have the relations

$$
\left\{\tilde{U}^{j}(\mathbf{x}), g^{i}(\mathbf{x})\right\}=0
$$

for $\mathbf{g}(\mathbf{x})=0$.

The functions $\tilde{U}^{j}(\mathbf{x})$ take the same values that $U^{j}(\mathbf{x})$ at the points of $\mathcal{N}^{k}$ and we can define the Dirac bracket $\{\cdot, \cdot\}_{D}$ on $\mathcal{N}^{k}$ by the formula

$$
\left\{U^{i}, U^{j}\right\}_{D}=\left.\left\{\tilde{U}^{i}(\mathbf{x}), \tilde{U}^{j}(\mathbf{x})\right\}\right|_{\mathcal{N}^{k}}(\mathbf{U}) .
$$

The functions $\beta_{s}^{j}(\mathbf{U})$ are determined from the linear systems

$$
\left.\left\{g^{i}(\mathbf{x}), g^{s}(\mathbf{x})\right\}\right|_{\mathcal{N}^{k}} \beta_{s}^{j}(\mathbf{U})+\left.\left\{g^{i}(\mathbf{x}), U^{j}(\mathbf{x})\right\}\right|_{\mathcal{N}^{k}}=0, \quad i=1, \ldots, n-k,
$$

and we can also write

$$
\left\{U^{i}, U^{j}\right\}_{D}=\left.\left\{U^{i}(\mathbf{x}), U^{j}(\mathbf{x})\right\}\right|_{\mathcal{N}^{k}}-\left.\beta_{s}^{i}(\mathbf{U})\left\{g^{s}(\mathbf{x}), g^{q}(\mathbf{x})\right\}\right|_{\mathcal{N}^{k}} \beta_{q}^{j}(\mathbf{U})
$$

for the Dirac bracket on $\mathcal{N}^{k}$. 
We now describe the procedure of the Dirac restriction, which will be needed in our situation. We consider now system (2.3) which is Hamiltonian with respect to some local bracket (2.16) with a local Hamiltonian function of the form (2.17). We first introduce the extended space of fields

$$
\varphi(x) \rightarrow \varphi(\theta, x),
$$

where the functions $\boldsymbol{\varphi}(\boldsymbol{\theta}, x)$ are $2 \pi$-periodic with respect to each $\theta^{\alpha}$, and define the extended Poisson bracket

$$
\left\{\varphi^{i}(\boldsymbol{\theta}, x), \varphi^{j}\left(\boldsymbol{\theta}^{\prime}, y\right)\right\}=\sum_{k \geq 0} B_{(k)}^{i j}\left(\boldsymbol{\varphi}, \boldsymbol{\varphi}_{x}, \ldots\right) \delta^{(k)}(x-y) \delta\left(\boldsymbol{\theta}-\boldsymbol{\theta}^{\prime}\right) .
$$

We also make the replacement $x \rightarrow X=\epsilon x$ and define the Poisson bracket

$$
\left\{\varphi^{i}(\boldsymbol{\theta}, X), \varphi^{j}\left(\boldsymbol{\theta}^{\prime}, Y\right)\right\}=\sum_{k \geq 0} \epsilon^{k} B_{(k)}^{i j}\left(\boldsymbol{\varphi}, \epsilon \boldsymbol{\varphi}_{X}, \ldots\right) \delta^{(k)}(X-Y) \delta\left(\boldsymbol{\theta}-\boldsymbol{\theta}^{\prime}\right)
$$

on the space of fields $\varphi(\theta, X)$.

Let us define again the submanifold $\mathcal{K}$ by the formula (2.12) in the extended space of fields. We thus assume that the functions $\boldsymbol{\varphi}(\boldsymbol{\theta}, X)$ represent functions from the family $\Lambda$ of the $m$-phase solutions of (2.3) with some parameters $\mathbf{U}(X)$ at every $X$.

It is convenient to choose the boundary conditions in the form $\mathbf{U}(X) \rightarrow \mathbf{U}_{0}, X \rightarrow \pm \infty$, such that $k^{\alpha}\left(\mathbf{U}_{0}\right)=0$ and define the functions $S^{\alpha}(X)$ by the formula

$$
S^{\alpha}(X)=\frac{1}{2} \int_{-\infty}^{+\infty} \operatorname{sgn}(X-Y) k^{\alpha}(Y) \mathrm{d} Y .
$$

We can write then

$$
\varphi^{i}(\boldsymbol{\theta}, X)=\Phi^{i}\left(\frac{\mathbf{S}[\mathbf{U}](X)}{\epsilon}+\boldsymbol{\theta}, \mathbf{U}(X)\right)
$$

where $S^{\alpha}[\mathbf{U}]$ is a single-valued functional of coordinates $\mathbf{U}(X)$ on the submanifold $\mathcal{K}{ }^{2}$

Let us introduce the functionals $J^{\nu}(X)$ on the functions $\varphi(\theta, X)$ by the formula

$$
J^{\nu}(X)=\int_{0}^{2 \pi} \cdots \int_{0}^{2 \pi} P^{\nu}\left(\varphi, \epsilon \varphi_{X}, \epsilon^{2} \varphi_{X X}, \ldots\right) \frac{\mathrm{d}^{m} \theta}{(2 \pi)^{m}}
$$

and consider their values on the functions of the family $\mathcal{K}$.

We can write on $\mathcal{K}$,

$$
J^{\nu}(X)=U^{\nu}(X)+\sum_{l \geq 1} \epsilon^{l} J_{(l)}^{\nu}(X)
$$

where $J_{(l)}^{\nu}(X)$ are polynomials in the derivatives $\mathbf{U}_{X}, \mathbf{U}_{X X}, \ldots$ with coefficients depending on $\mathbf{U}$ and have grading degree $l$ in terms of total number of derivations with respect to $X$.

The transformation (2.30) can be inverted as a formal series in $\epsilon$, such that we can write

$$
U^{\nu}(X)=J^{\nu}(X)+\sum_{l \geq 1} \epsilon^{l} U_{(l)}^{\nu}(X)
$$

\footnotetext{
${ }^{2}$ The case when the values $k^{\alpha}=0$ are absent on the space of the parameters $\mathbf{U}$ requires just a simple modification of the definition of $\mathbf{S}(X)$ which we do not consider here.
} 
on the functions of the submanifold $\mathcal{K}$. In the formula (2.31) the functions $U_{(l)}^{\nu}$ are functions of $\mathbf{J}, \mathbf{J}_{X}, \mathbf{J}_{X X}, \ldots$, polynomial in the derivatives $\mathbf{J}_{X}, \mathbf{J}_{X X}, \ldots$, and having degree $l$ in terms of the number of derivations w.r.t. $X$.

The values of the functionals $J^{\nu}(X)$ or $U^{\nu}(X)$ on the functions from $\mathcal{K}$ can thus be chosen as coordinates on $\mathcal{K}$. We can define also the functionals $\mathbf{U}(X)$ on the whole functional space using the definition of the functionals $\mathbf{J}(X)$ and relations (2.31). Let us introduce also the constraints $g^{i}(\boldsymbol{\theta}, X)$ defining the submanifold $\mathcal{K}$ by the conditions $g^{i}(\boldsymbol{\theta}, X)=0$ and numbered by the values of $\boldsymbol{\theta}$ and $X$

$$
g^{i}(\boldsymbol{\theta}, X)=\varphi^{i}(\boldsymbol{\theta}, X)-\Phi^{i}\left(\frac{\mathbf{S}[\mathbf{U}[\mathbf{J}]](X)}{\epsilon}+\boldsymbol{\theta}, \mathbf{U}[\mathbf{J}](X)\right) .
$$

The constraints $g^{i}(\boldsymbol{\theta}, X)$ are functionals on the whole extended space of fields $\varphi^{i}(\boldsymbol{\theta}, X)$ by virtue of the corresponding definition of the functionals $J^{\nu}(X)$.

The constraints $(2.32)$ are not independent since the following relations hold identically for the "gradients" $\delta g^{i}(\boldsymbol{\theta}, X) / \delta \varphi^{j}\left(\boldsymbol{\theta}^{\prime}, Y\right)$ on the submanifold $\mathcal{K}$

$$
\left.\left.\int_{-\infty}^{+\infty} \int_{0}^{2 \pi} \cdots \int_{0}^{2 \pi} \frac{\delta J^{\nu}(Z)}{\delta \varphi^{i}(\boldsymbol{\theta}, X)}\right|_{\mathcal{K}} \frac{\delta g^{i}(\boldsymbol{\theta}, X)}{\delta \varphi^{j}\left(\boldsymbol{\theta}^{\prime}, Y\right)}\right|_{\mathcal{K}} \frac{\mathrm{d}^{m} \theta}{(2 \pi)^{m}} \mathrm{~d} X \equiv 0
$$

Nevertheless, it will be convenient here not to choose an independent subsystem from (2.32) and keep the system of constraints in the form (2.32) keeping in mind the existence of identities (2.33).

Thus, we consider now the values of the functionals $[\mathbf{U}(X), \mathbf{g}(\boldsymbol{\theta}, X)]$ as a coordinate system in the neighborhood of $\mathcal{K}$ with the relations (2.33). The values of the functionals $\mathbf{U}(X)$ will be considered as a coordinate system on $\mathcal{K}$.

Using the explicit expression for the quantities $\delta J^{\nu}(Z) / \delta \varphi^{i}(\boldsymbol{\theta}, X)$ on $\mathcal{K}$

$$
\begin{aligned}
& \left.\frac{\delta J^{\nu}(Z)}{\delta \varphi^{i}(\boldsymbol{\theta}, X)}\right|_{\mathcal{K}} \\
& \quad=\sum_{l \geq 0} \Pi_{i(l)}^{\nu}\left(\boldsymbol{\Phi}\left(\frac{\mathbf{S}(Z)}{\epsilon}+\boldsymbol{\theta}, \mathbf{U}(Z)\right), \epsilon \frac{\partial}{\partial Z} \boldsymbol{\Phi}\left(\frac{\mathbf{S}(Z)}{\epsilon}+\boldsymbol{\theta}, \mathbf{U}(Z)\right), \ldots\right) \epsilon^{l} \delta^{(l)}(Z-X),
\end{aligned}
$$

we can write the convolution (2.33) in the form of action of the operator

$$
\int_{0}^{2 \pi} \cdots \int_{0}^{2 \pi} \frac{\mathrm{d}^{m} \theta}{(2 \pi)^{m}} \sum_{l \geq 0} \Pi_{i(l)}^{\nu}\left(\boldsymbol{\Phi}\left(\frac{\mathbf{S}(Z)}{\epsilon}+\boldsymbol{\theta}, \mathbf{U}(Z)\right), \epsilon \frac{\partial}{\partial Z} \boldsymbol{\Phi}\left(\frac{\mathbf{S}(Z)}{\epsilon}+\boldsymbol{\theta}, \mathbf{U}(Z)\right), \ldots\right) \epsilon^{l} \frac{\mathrm{d}^{l}}{\mathrm{~d} Z^{l}}
$$

on the distributions $\delta g^{i}(\boldsymbol{\theta}, Z) /\left.\delta \varphi^{j}\left(\boldsymbol{\theta}^{\prime}, Y\right)\right|_{\mathcal{K}}$.

Pairwise Poisson brackets of the functionals $J^{\nu}(X), J^{\mu}(Y)$ have the form

$$
\left\{J^{\nu}(X), J^{\mu}(Y)\right\}=\sum_{k \geq 0} \int_{0}^{2 \pi} \cdots \int_{0}^{2 \pi} A_{k}^{\nu \mu}\left(\boldsymbol{\varphi}(\boldsymbol{\theta}, X), \epsilon \boldsymbol{\varphi}_{X}(\boldsymbol{\theta}, X), \ldots\right) \frac{\mathrm{d}^{m} \theta}{(2 \pi)^{m}} \epsilon^{k} \delta^{(k)}(X-Y),
$$

where

$$
A_{0}^{\nu \mu}\left(\boldsymbol{\varphi}, \epsilon \boldsymbol{\varphi}_{X}, \ldots\right) \equiv \epsilon \partial_{X} Q^{\nu \mu}\left(\boldsymbol{\varphi}, \epsilon \boldsymbol{\varphi}_{X}, \ldots\right) .
$$

Substituting the functions $\varphi^{i}(\boldsymbol{\theta}, X)$ in the form (2.28) it is easy to see that the DubrovinNovikov bracket is the main part (in $\epsilon$ ) of the pairwise Poisson brackets $\left\{J^{\nu}(X), J^{\mu}(Y)\right\}$ on the submanifold $\mathcal{K}$ in the coordinates $[\mathbf{U}(Z)]$. 
The Poisson brackets of the fields $\varphi^{i}(\boldsymbol{\theta}, X)$ with the functionals $J^{\mu}(Y)$ can be written as

$$
\left\{\varphi^{i}(\boldsymbol{\theta}, X), J^{\mu}(Y)\right\}=\sum_{l \geq 0} \epsilon^{l} C_{(l)}^{i \mu}\left(\boldsymbol{\varphi}(\boldsymbol{\theta}, X), \epsilon \boldsymbol{\varphi}_{X}(\boldsymbol{\theta}, X), \ldots\right) \delta^{(l)}(X-Y)
$$

with some smooth functions $C_{(l)}^{i \mu}\left(\varphi, \epsilon \varphi_{X}, \ldots\right)$.

We also have in this case

$$
C_{(0)}^{i \mu}\left(\boldsymbol{\varphi}(\boldsymbol{\theta}, X), \epsilon \boldsymbol{\varphi}_{X}(\boldsymbol{\theta}, X), \ldots\right) \equiv S^{i \mu}\left(\boldsymbol{\varphi}(\boldsymbol{\theta}, X), \epsilon \boldsymbol{\varphi}_{X}(\boldsymbol{\theta}, X), \ldots\right)
$$

by virtue of $(2.23)$.

For any function of the slow variable $q(Y)$ we can write

$$
\left\{\varphi^{i}(\boldsymbol{\theta}, X), \int q(Y) J^{\mu}(Y) \mathrm{d} Y\right\}=\sum_{l \geq 0} \epsilon^{l} C_{(l)}^{i \mu}\left(\boldsymbol{\varphi}(\boldsymbol{\theta}, X), \epsilon \boldsymbol{\varphi}_{X}(\boldsymbol{\theta}, X), \ldots\right) q_{l X} .
$$

The leading term in the expression $(2.38)$ on $\mathcal{K}$ has the form

$$
\begin{aligned}
\left.\left\{\varphi^{i}(\boldsymbol{\theta}, X), \int q(Y) J^{\mu}(Y) \mathrm{d} Y\right\}\right|_{\mathcal{K}[0]} & \\
= & C_{(0)}^{i \mu}\left(\boldsymbol{\Phi}\left(\frac{\mathbf{S}(X)}{\epsilon}+\boldsymbol{\theta}, X\right), k^{\beta} \mathbf{\Phi}_{\theta^{\beta}}\left(\frac{\mathbf{S}(X)}{\epsilon}+\boldsymbol{\theta}, X\right), \ldots\right) q(X) \\
= & q(X) S^{i \mu}\left(\boldsymbol{\Phi}\left(\frac{\mathbf{S}(X)}{\epsilon}+\boldsymbol{\theta}, X\right), k^{\beta} \mathbf{\Phi}_{\theta^{\beta}}\left(\frac{\mathbf{S}(X)}{\epsilon}+\boldsymbol{\theta}, X\right), \ldots\right),
\end{aligned}
$$

where $S^{i \mu}\left(\boldsymbol{\varphi}, \boldsymbol{\varphi}_{x}, \ldots\right)$ is the flow (2.23) generated by the functional $I^{\mu}$. According to (2.24) we can write then

$$
\left.\left\{\varphi^{i}(\boldsymbol{\theta}, X), \int q(Y) J^{\mu}(Y) \mathrm{d} Y\right\}\right|_{\mathcal{K}}=\omega^{\alpha \mu}(X) \Phi_{\theta^{\alpha}}^{i}\left(\frac{\mathbf{S}(X)}{\epsilon}+\boldsymbol{\theta}, \mathbf{U}(X)\right) q(X)+O(\epsilon) .
$$

Similarly, for any smooth function $Q(\boldsymbol{\theta}, X), 2 \pi$-periodic in each $\theta^{\alpha}$, we can write on the basis of $(2.36)$

$$
\begin{aligned}
\int_{0}^{2 \pi} & \left.\cdots \int_{0}^{2 \pi} Q\left(\frac{\mathbf{S}(X)}{\epsilon}+\boldsymbol{\theta}, X\right)\left\{\varphi^{i}(\boldsymbol{\theta}, X), J^{\mu}(Y)\right\}\right|_{\mathcal{K}} \frac{\mathrm{d}^{m} \theta}{(2 \pi)^{m}} \\
& =\omega^{\alpha \mu}(X) \int_{0}^{2 \pi} \cdots \int_{0}^{2 \pi} Q(\boldsymbol{\theta}, X) \Phi_{\theta^{\alpha}}^{i}(\boldsymbol{\theta}, \mathbf{U}(X)) \frac{\mathrm{d}^{m} \theta}{(2 \pi)^{m}} \delta(X-Y)+O(\epsilon) .
\end{aligned}
$$

In view of relations $(2.30),(2.31)$ for the functionals $J^{\mu}(Y), U^{\mu}(Y)$ we can also write

$$
\begin{gathered}
\left.\left\{\varphi^{i}(\boldsymbol{\theta}, X), \int q(Y) U^{\mu}(Y) \mathrm{d} Y\right\}\right|_{\mathcal{K}}=\omega^{\alpha \mu}(X) \Phi_{\theta^{\alpha}}^{i}\left(\frac{\mathbf{S}(X)}{\epsilon}+\boldsymbol{\theta}, \mathbf{U}(X)\right) q(X)+O(\epsilon), \\
\left.\int_{0}^{2 \pi} \cdots \int_{0}^{2 \pi} Q\left(\frac{\mathbf{S}(X)}{\epsilon}+\boldsymbol{\theta}, X\right)\left\{\varphi^{i}(\boldsymbol{\theta}, X), U^{\mu}(Y)\right\}\right|_{\mathcal{K}} \frac{\mathrm{d}^{m} \theta}{(2 \pi)^{m}} \\
=\omega^{\alpha \mu}(X) \int_{0}^{2 \pi} \cdots \int_{0}^{2 \pi} Q(\boldsymbol{\theta}, X) \Phi_{\theta^{\alpha}}^{i}(\boldsymbol{\theta}, \mathbf{U}(X)) \frac{\mathrm{d}^{m} \theta}{(2 \pi)^{m}} \delta(X-Y)+O(\epsilon) .
\end{gathered}
$$

In this case, by virtue of $(2.25)$ we have

$$
\left.\left\{\varphi^{i}(\boldsymbol{\theta}, X), \int q(Y) k^{\alpha}(\mathbf{U}(Y)) \mathrm{d} Y\right\}\right|_{\mathcal{K}}=O(\epsilon)
$$




$$
\left.\int_{0}^{2 \pi} \cdots \int_{0}^{2 \pi} Q\left(\frac{\mathbf{S}(X)}{\epsilon}+\boldsymbol{\theta}, X\right)\left\{\varphi^{i}(\boldsymbol{\theta}, X), k^{\alpha}(\mathbf{U}(Y))\right\}\right|_{\mathcal{K}} \frac{\mathrm{d}^{m} \theta}{(2 \pi)^{m}}=O(\epsilon)
$$

for fixed values of coordinates $[\mathbf{U}(Z)]$.

Let us note here that since we consider the change of coordinates, which depends explicitly on $\epsilon$, we shall assume now, that all relations are written in the coordinate system $[\mathbf{U}(X), \mathbf{g}(\boldsymbol{\theta}, X)]$ near $\mathcal{K}$. In particular, all the values on $\mathcal{K}$ will be functionals of $\mathbf{U}(X)$, represented usually in the form of graded expansions in $\epsilon$ with coefficients depending on $\mathbf{U}, \mathbf{U}_{X}, \mathbf{U}_{X X}, \ldots$

Let us prove here some lemmas about the structure of Poisson brackets on the submanifold $\mathcal{K}$ which we will need in the further consideration.

Lemma 2.3. Let the values $U^{\nu}$ of the functionals $I^{\nu}$ on a complete regular family $\Lambda$ of m-phase solutions of (2.3) be functionally independent and give a complete set of parameters on $\Lambda$, excluding the initial phases. Then for the Poisson brackets of the functionals $k^{\alpha}(\mathbf{U}(X))$ and $J^{\mu}(Y)$ on $\mathcal{K}$ we have the following relation

$$
\left.\left\{k^{\alpha}(\mathbf{U}(X)), J^{\mu}(Y)\right\}\right|_{\mathcal{K}}=\epsilon\left[\omega^{\alpha \mu}(\mathbf{U}(X)) \delta(X-Y)\right]_{X}+O\left(\epsilon^{2}\right) .
$$

Proof. The conditions of Lemma 2.3 coincide with the conditions of Lemmas 2.1 and 2.2. Consider the Hamiltonian flow generated by the functional $\int q(Y) J^{\mu}(Y) \mathrm{d} Y$ according to the bracket (2.27) with a compactly supported function $q(Y)$ of the slow variable $Y=\epsilon y$.

According to $(2.38)$ we can write at the "points" of the submanifold $\mathcal{K}$

$$
\varphi_{t}^{i}=q(X) \omega^{\beta \mu}(\mathbf{U}(X)) \Phi_{\theta^{\beta}}^{i}\left(\frac{\mathbf{S}(X)}{\epsilon}+\boldsymbol{\theta}, \mathbf{U}(X)\right)+\epsilon \eta_{[q]}^{i}\left(\frac{\mathbf{S}(X)}{\epsilon}+\boldsymbol{\theta}, X\right)+O\left(\epsilon^{2}\right)
$$

with some $\left(2 \pi\right.$-periodic in each $\left.\theta^{\alpha}\right)$ functions $\eta_{[q]}^{i}(\boldsymbol{\theta}, X)$.

Let us introduce the functionals $k^{\alpha}(\mathbf{J}(X))=k^{\alpha}\left(J^{1}(X), \ldots, J^{N}(X)\right)$ using the same functions $k^{\alpha}(\mathbf{U})$. According to $(2.30),(2.31)$ we can write

$$
\left.\left\{k^{\alpha}(\mathbf{U}(X)), J^{\mu}(Y)\right\}\right|_{\mathcal{K}}=\left.\left\{k^{\alpha}(\mathbf{J}(X)), J^{\mu}(Y)\right\}\right|_{\mathcal{K}}+O\left(\epsilon^{2}\right) .
$$

Consider the evolution of the functionals $k^{\alpha}(\mathbf{J}(X))$ according to the flow generated by $\int q(Y) J^{\mu}(Y) \mathrm{d} Y$ on the submanifold $\mathcal{K}$. Using relations $(2.46)$ we can write

$$
\begin{aligned}
& k_{t}^{\alpha}(\mathbf{J}(X))=\frac{\partial k^{\alpha}}{\partial U^{\nu}}(\mathbf{J}(X)) J_{t}^{\nu}(X)=\frac{\partial k^{\alpha}}{\partial U^{\nu}}(\mathbf{J}(X)) q(X) \omega^{\beta \mu}(\mathbf{U}(X)) \\
& \times \int_{0}^{2 \pi} \cdots \int_{0}^{2 \pi} \sum_{l \geq 0} \Pi_{i(l)}^{\nu}\left(\boldsymbol{\Phi}\left(\frac{\mathbf{S}(X)}{\epsilon}+\boldsymbol{\theta}, \mathbf{U}(X)\right), \epsilon \frac{\partial}{\partial X} \boldsymbol{\Phi}\left(\frac{\mathbf{S}(X)}{\epsilon}+\boldsymbol{\theta}, \mathbf{U}(X)\right), \ldots\right) \\
& \quad \times \epsilon^{l} \frac{\partial^{l}}{\partial X^{l}} \Phi_{\theta^{\beta}}^{i}\left(\frac{\mathbf{S}(X)}{\epsilon}+\boldsymbol{\theta}, \mathbf{U}(X)\right) \frac{\mathrm{d}^{m} \theta}{(2 \pi)^{m}} \\
& +\epsilon \frac{\partial k^{\alpha}}{\partial U^{\nu}}(\mathbf{U}(X))\left(q(X) \omega^{\beta \mu}(\mathbf{U}(X))\right)_{X} \\
& \times \int_{0}^{2 \pi} \ldots \int_{0}^{2 \pi} \sum_{l \geq 1} l \Pi_{i(l)}^{\nu}\left(\mathbf{\Phi}(\boldsymbol{\theta}, \mathbf{U}(X)), k^{\gamma} \mathbf{\Phi}_{\theta^{\gamma}}(\boldsymbol{\theta}, \mathbf{U}(X)), \ldots\right) \\
& \times k^{\alpha_{1}}(X) \cdots k^{\alpha_{l-1}}(X) \Phi_{\theta^{\beta} \theta^{\alpha_{1} \ldots \theta^{\alpha} l-1}}(\boldsymbol{\theta}, \mathbf{U}(X)) \frac{\mathrm{d}^{m} \theta}{(2 \pi)^{m}} \\
& +\epsilon \frac{\partial k^{\alpha}}{\partial U^{\nu}}(\mathbf{U}(X)) \int_{0}^{2 \pi} \ldots \int_{0}^{2 \pi} \sum_{l \geq 0} \Pi_{i(l)}^{\nu}\left(\boldsymbol{\Phi}(\boldsymbol{\theta}, \mathbf{U}(X)), k^{\gamma} \mathbf{\Phi} \theta^{\gamma}(\boldsymbol{\theta}, \mathbf{U}(X)), \ldots\right) \\
& \times k^{\alpha_{1}}(X) \cdots k^{\alpha_{l}}(X) \eta_{[q] \theta^{\alpha_{1} \ldots} \theta^{\alpha_{l}}}^{i}(\boldsymbol{\theta}, X) \frac{\mathrm{d}^{m} \theta}{(2 \pi)^{m}}+O\left(\epsilon^{2}\right) .
\end{aligned}
$$


It's not difficult to see that the first part of the above expression contains the integrals over $\boldsymbol{\theta}$ of the expressions $P_{\theta^{\beta}}^{\nu}(\boldsymbol{\theta}, X)$ and is equal to zero. It is easy to see also after integration by parts that the third part of the above expression represents the value

$$
\epsilon \int_{0}^{2 \pi} \cdots \int_{0}^{2 \pi} \frac{\partial k^{\alpha}}{\partial U^{\nu}}(\mathbf{U}(X)) \zeta_{i[\mathbf{U}(X)]}^{(\nu)}(\boldsymbol{\theta}) \eta_{[q]}^{i}(\boldsymbol{\theta}, X) \frac{\mathrm{d}^{m} \theta}{(2 \pi)^{m}}
$$

and is equal to zero according to Lemma 2.1.

Thus, we can write in the main order the expressions for the evolution of the functionals $k^{\alpha}(\mathbf{J}(X))$ on the submanifold $\mathcal{K}$ in the form

$$
\begin{aligned}
& k_{t}^{\alpha}(\mathbf{J}(X))=\epsilon\left(q(X) \omega^{\beta \mu}(\mathbf{U}(X))\right)_{X} \\
& \quad \times \frac{\partial k^{\alpha}}{\partial U^{\nu}} \int_{0}^{2 \pi} \cdots \int_{0}^{2 \pi} \sum_{l \geq 1} l k^{\beta_{1}} \cdots k^{\beta_{l-1}} \Phi_{\theta^{\beta} \theta^{\beta_{1} \ldots \theta^{\beta_{l-1}}}}^{i} \Pi_{i(l)}^{\nu}\left(\boldsymbol{\Phi}, k^{\gamma} \mathbf{\Phi}_{\theta^{\gamma}}, \ldots\right) \frac{\mathrm{d}^{m} \theta}{(2 \pi)^{m}}+O\left(\epsilon^{2}\right) .
\end{aligned}
$$

Using relations (2.13) we get then

$$
k_{t}^{\alpha}(\mathbf{J}(X))=\epsilon\left[q(X) \omega^{\alpha \mu}(\mathbf{U}(X))\right]_{X}+O\left(\epsilon^{2}\right),
$$

i.e.

$$
\left.\left\{k^{\alpha}(\mathbf{J}(X)), J^{\mu}(Y)\right\}\right|_{\mathcal{K}}=\epsilon \omega^{\alpha \mu}(\mathbf{U}(X)) \delta^{\prime}(X-Y)+\epsilon \omega_{X}^{\alpha \mu} \delta(X-Y)+O\left(\epsilon^{2}\right),
$$

which implies (2.45).

Easy to see that according to Lemma 2.3 and relations $(2.30),(2.31)$ we can write also

$$
\left.\left\{k^{\alpha}(\mathbf{U}(X)), U^{\mu}(Y)\right\}\right|_{\mathcal{K}}=\epsilon\left[\omega^{\alpha \mu}(\mathbf{U}(X)) \delta(X-Y)\right]_{X}+O\left(\epsilon^{2}\right)
$$

on the submanifold $\mathcal{K}$.

Corollary 2.1. Under the conditions of Lemma 2.3, we have also

$$
\left.\left\{k^{\alpha}(X), k^{\beta}(Y)\right\}\right|_{\mathcal{K}}=O\left(\epsilon^{2}\right)
$$

for the functionals $\mathbf{k}(\mathbf{U}(X))$.

Indeed, by virtue of (2.25) we have

$$
\begin{aligned}
\left.\left\{k^{\alpha}(X), k^{\beta}(Y)\right\}\right|_{\mathcal{K}}= & \left.\left\{k^{\alpha}(X), U^{\mu}(Y)\right\}\right|_{\mathcal{K}} \frac{\partial k^{\beta}}{\partial U^{\mu}}(\mathbf{U}(Y)) \\
= & \epsilon \omega^{\alpha \mu}(X) k_{U^{\mu}}^{\beta}(X) \delta^{\prime}(X-Y)+\epsilon \omega^{\alpha \mu}(X)\left(k_{U^{\mu}}^{\beta}(X)\right)_{X} \delta(X-Y) \\
& +\epsilon\left(\omega^{\alpha \mu}(X)\right)_{X} k_{U^{\mu}}^{\beta}(X) \delta(X-Y)+O\left(\epsilon^{2}\right)=O\left(\epsilon^{2}\right) .
\end{aligned}
$$

Let us note now that in the proof of Lemma 2.3, we have not used the fact that the functionals $J^{\mu}(Y)$ belong to our special set of functionals (2.29) and used only the fact that the flow generated by the functional $I^{\mu}$ leaves invariant the family $\Lambda$ generating linear shifts of $\theta_{0}^{\alpha}$ with constant frequencies $\omega^{\alpha \mu}(\mathbf{U})$. We can therefore formulate here the following lemma.

Lemma 2.3'. Let the values $U^{\nu}$ of the functionals $I^{\nu}$ on a complete regular family $\Lambda$ of m-phase solutions of system (2.3) be functionally independent and give a complete set of parameters on $\Lambda$, excluding the initial phases. Let the flow generated by the functional

$$
\tilde{I}=\int \tilde{P}\left(\boldsymbol{\varphi}, \boldsymbol{\varphi}_{x}, \ldots\right) \mathrm{d} x
$$

leave invariant the family $\Lambda$ generating a linear shift of $\theta_{0}^{\alpha}$ with constant frequencies $\tilde{\omega}^{\alpha}(\mathbf{U})$. 
Consider the functionals

$$
\tilde{J}(X)=\int_{0}^{2 \pi} \cdots \int_{0}^{2 \pi} \tilde{P}\left(\boldsymbol{\varphi}, \epsilon \boldsymbol{\varphi}_{X}, \epsilon^{2} \boldsymbol{\varphi}_{X X}, \ldots\right) \frac{\mathrm{d}^{m} \theta}{(2 \pi)^{m}}
$$

Then for the Poisson brackets of the functionals $k^{\alpha}(\mathbf{U}(X))$ and $\tilde{J}(Y)$ on $\mathcal{K}$ we have the relation

$$
\left.\left\{k^{\alpha}(\mathbf{U}(X)), \tilde{J}(Y)\right\}\right|_{\mathcal{K}}=\epsilon\left[\tilde{\omega}^{\alpha}(\mathbf{U}(X)) \delta(X-Y)\right]_{X}+O\left(\epsilon^{2}\right) .
$$

Proof of Lemma 2.3' completely repeats the proof of Lemma 2.3.

Lemma 2.4. Let the values $U^{\nu}$ of the functionals $I^{\nu}$ on a complete regular family $\Lambda$ of $m$-phase solutions of system (2.3) be functionally independent and give a complete set of parameters on $\Lambda$, excluding the initial phases. Then for the constraints $g^{i}(\boldsymbol{\theta}, X)$ imposed by (2.32) and smooth compactly supported function $q(X)$ as well as smooth $2 \pi$-periodic in each $\theta^{\alpha}$ function $Q(\boldsymbol{\theta}, X)$ we have the following relations on the submanifold $\mathcal{K}$

$$
\begin{aligned}
& \left.\left\{g^{i}(\boldsymbol{\theta}, X), \int q(Y) J^{\mu}(Y) \mathrm{d} Y\right\}\right|_{\mathcal{K}}=O(\epsilon), \\
& {\left.\left[\int_{0}^{2 \pi} \cdots \int_{0}^{2 \pi} Q\left(\frac{\mathbf{S}(X)}{\epsilon}+\boldsymbol{\theta}, X\right)\left\{g^{i}(\boldsymbol{\theta}, X), J^{\mu}(Y)\right\} \frac{\mathrm{d}^{m} \theta}{(2 \pi)^{m}}\right]\right|_{\mathcal{K}}=O(\epsilon) .}
\end{aligned}
$$

Proof. Indeed, by (2.39), (2.40), and Lemma 2.3 we have

$$
\begin{aligned}
& \left.\left\{g^{i}(\boldsymbol{\theta}, X), \int q(Y) J^{\mu}(Y) \mathrm{d} Y\right\}\right|_{\mathcal{K}[0]}=\Phi_{\theta^{\alpha}}^{i}\left(\frac{\mathbf{S}(X)}{\epsilon}+\boldsymbol{\theta}, \mathbf{U}(X)\right) \omega^{\alpha \mu}(X) q(X) \\
& -\frac{1}{2} \Phi_{\theta^{\alpha}}^{i}\left(\frac{\mathbf{S}(X)}{\epsilon}+\boldsymbol{\theta}, \mathbf{U}(X)\right) \int \operatorname{sgn}(X-Z)\left(\omega^{\alpha \mu}(Z) q(Z)\right)_{Z} \mathrm{~d} Z \equiv 0, \\
& {\left.\left[\int_{0}^{2 \pi} \cdots \int_{0}^{2 \pi} Q\left(\frac{\mathbf{S}(X)}{\epsilon}+\boldsymbol{\theta}, X\right)\left\{g^{i}(\boldsymbol{\theta}, X), J^{\mu}(Y)\right\} \frac{\mathrm{d}^{m} \theta}{(2 \pi)^{m}}\right]\right|_{\mathcal{K}[0]}} \\
& =\omega^{\alpha \mu}(X) \int_{0}^{2 \pi} \cdots \int_{0}^{2 \pi} Q(\boldsymbol{\theta}, X) \Phi_{\theta^{\alpha}}^{i}(\boldsymbol{\theta}, \mathbf{U}(X)) \frac{\mathrm{d}^{m} \theta}{(2 \pi)^{m}} \delta(X-Y) \\
& -\int_{0}^{2 \pi} \cdots \int_{0}^{2 \pi} Q(\boldsymbol{\theta}, X) \Phi_{\theta^{\alpha}}^{i}(\boldsymbol{\theta}, \mathbf{U}(X)) \frac{\mathrm{d}^{m} \theta}{(2 \pi)^{m}} \frac{1}{2} \operatorname{sgn}(X-Z)\left[\omega^{\alpha \mu}(Z) \delta(Z-Y)\right]_{Z} \mathrm{~d} Z \equiv 0 .
\end{aligned}
$$

Similarly to the previous case, we can formulate also the following lemma.

Lemma 2.4'. Let the values $U^{\nu}$ of the functionals $I^{\nu}$ on a complete regular family $\Lambda$ of $m$-phase solutions of system (2.3) be functionally independent and give a complete set of parameters on $\Lambda$, excluding the initial phases. Let the flow generated by the functional (2.49) leave invariant the family $\Lambda$ generating a linear shift of $\theta_{0}^{\alpha}$ with constant frequencies $\tilde{\omega}^{\alpha}(\mathbf{U})$. Then for the constraints $g^{i}(\boldsymbol{\theta}, X)$ and the functionals $\tilde{J}(X)$ imposed by (2.50) we have the following relations on the submanifold $\mathcal{K}$

$$
\begin{aligned}
& \left.\left\{g^{i}(\boldsymbol{\theta}, X), \int q(Y) \tilde{J}(Y) \mathrm{d} Y\right\}\right|_{\mathcal{K}}=O(\epsilon), \\
& {\left.\left[\int_{0}^{2 \pi} \cdots \int_{0}^{2 \pi} Q\left(\frac{\mathbf{S}(X)}{\epsilon}+\boldsymbol{\theta}, X\right)\left\{g^{i}(\boldsymbol{\theta}, X), \tilde{J}(Y)\right\} \frac{\mathrm{d}^{m} \theta}{(2 \pi)^{m}}\right]\right|_{\mathcal{K}}=O(\epsilon) .}
\end{aligned}
$$

Let us consider now the Dirac restriction of the bracket $(2.27)$ on the submanifold $\mathcal{K}$. 
For the Dirac restriction of the bracket $(2.27)$ on the submanifold $\mathcal{K}$ we have to modify the functionals $U^{\mu}(Y)$ by linear combinations of the constraints $g^{i}(\boldsymbol{\theta}, X)$

$$
\tilde{U}^{\mu}(Y)=U^{\mu}(Y)+\int_{-\infty}^{+\infty} \int_{0}^{2 \pi} \cdots \int_{0}^{2 \pi} g^{i}(\boldsymbol{\theta}, X) \alpha_{i}^{\mu}\left(\frac{\mathbf{S}[\mathbf{U}](X)}{\epsilon}+\boldsymbol{\theta},[\mathbf{U}], X, Y\right) \frac{\mathrm{d}^{m} \theta}{(2 \pi)^{m}} \mathrm{~d} X,
$$

so that the flows generated by the functionals $\tilde{U}^{\mu}(Y)$ leave $\mathcal{K}$ invariant and then use the functionals $\tilde{U}^{\mu}(Y)$ to construct the Dirac bracket on $\mathcal{K}$.

Since the functionals $U^{\mu}(Y)$ are defined with the aid of the functionals $\mathbf{J}(Z)$, technically it is more convenient to modify the functionals $J^{\mu}(Y)$

$$
\tilde{J}^{\mu}(Y)=J^{\mu}(Y)+\int_{-\infty}^{+\infty} \int_{0}^{2 \pi} \cdots \int_{0}^{2 \pi} g^{i}(\boldsymbol{\theta}, X) \beta_{i}^{\mu}\left(\frac{\mathbf{S}[\mathbf{U}[\mathbf{J}]](X)}{\epsilon}+\boldsymbol{\theta},[\mathbf{U}[\mathbf{J}]], X, Y\right) \frac{\mathrm{d}^{m} \theta}{(2 \pi)^{m}} \mathrm{~d} X,
$$

so that the flows generated by the functionals $\tilde{J}^{\mu}(Y)$ leave $\mathcal{K}$ invariant and then put

$$
\tilde{U}^{\mu}(Y)=\tilde{J}^{\mu}(Y)+\sum_{l \geq 1} \epsilon^{l} \tilde{U}_{(l)}^{\mu}(Y)
$$

just using the functionals $\tilde{\mathbf{J}}(Y)$ instead of $\mathbf{J}(Y)$ in (2.31). Both the approaches give, certainly, the same result for the Dirac restriction of the bracket $(2.27)$ on $\mathcal{K}$.

The distributions $\beta_{i}^{\mu}(\mathbf{S}(X) / \epsilon+\boldsymbol{\theta}, X, Y)$ must satisfy the relation

$$
\begin{aligned}
& \int_{-\infty}^{+\infty} \int_{0}^{2 \pi} \cdots \int_{0}^{2 \pi}\left.\left\{g^{i}(\boldsymbol{\theta}, X), g^{j}\left(\boldsymbol{\theta}^{\prime}, Z\right)\right\}\right|_{\mathcal{K}} \beta_{j}^{\mu}\left(\frac{\mathbf{S}(Z)}{\epsilon}+\boldsymbol{\theta}^{\prime}, Z, Y\right) \frac{\mathrm{d}^{m} \theta^{\prime}}{(2 \pi)^{m}} \mathrm{~d} Z \\
&+\left.\left\{g^{i}(\boldsymbol{\theta}, X), J^{\mu}(Y)\right\}\right|_{\mathcal{K}}=0
\end{aligned}
$$

on $\mathcal{K}$ and are defined at each "point" of $\mathcal{K}$ up to linear combinations of the distributions $\delta J^{\zeta}(W) / \delta \varphi^{i}(\boldsymbol{\theta}, X)$ by virtue of the original dependence (2.33) of the constraints (2.32).

To obtain a local Poisson bracket on $\mathcal{K}$ we are trying to find the functions $\beta_{i}^{\mu}(\boldsymbol{\theta}, X, Y)$ in the form

$$
\beta_{i}^{\mu}(\boldsymbol{\theta}, X, Y)=\sum_{l \geq 1} \epsilon^{l} \beta_{i(l)}^{\mu}(\boldsymbol{\theta}, X, Y),
$$

where the functions $\beta_{i(l)}^{\mu}(\boldsymbol{\theta}, X, Y)$ represent the local distributions

$$
\beta_{i(l)}^{\mu}(\boldsymbol{\theta}, X, Y)=\sum_{p=0}^{l} \beta_{i(l), p}^{\mu}(\boldsymbol{\theta}, X) \delta^{(p)}(X-Y)
$$

with the total grading $l$ in $\epsilon$, considering that the derivatives of the delta function $\delta^{(p)}(X-Y)$ have by definition the degree $p$.

Thus, we assume that all the functions $\beta_{i(l), p}^{\mu}(\boldsymbol{\theta}, X)$ on $\mathcal{K}$ are local functionals of $\left(\mathbf{U}(X), \mathbf{U}_{X}\right.$, $\ldots)$ at every $\boldsymbol{\theta}$, polynomial in the derivatives $\left(\mathbf{U}_{X}, \ldots\right)$ and having degree $l-p$ under the previous definition. Such a structure of $\beta_{i}^{\mu}(\boldsymbol{\theta}, X, Y)$ is obviously equivalent to saying that the functionals

$$
J_{[\mathbf{q}]}=\int_{-\infty}^{+\infty} J^{\mu}(Y) q_{\mu}(Y) \mathrm{d} Y
$$

can be modified by linear combinations of constraints (2.32) with the coefficients

$$
B_{i[\mathbf{q}]}(\boldsymbol{\theta}, X)=\sum_{l \geq 1} \epsilon^{l} B_{i[\mathbf{q}](l)}(\boldsymbol{\theta}, X)=\sum_{l \geq 1} \epsilon^{l} \sum_{p=0}^{l} \beta_{i(l), p}^{\mu}(\boldsymbol{\theta}, X) \frac{\mathrm{d}^{p} q_{\mu}(X)}{\mathrm{d} X^{p}},
$$


so that the corresponding flows leave invariant the submanifold $\mathcal{K}$. Under this scheme, the derivatives $\mathrm{d}^{p} q_{\mu} / \mathrm{d} X^{p}$ of slowly varying functions $q_{\mu}(X)$ also have grading $p$ as the derivatives of the functionals $\mathbf{U}(X)$.

The pairwise Poisson brackets of the constraints $g^{i}(\boldsymbol{\theta}, X), g^{j}\left(\boldsymbol{\theta}^{\prime}, Y\right)$ on $\mathcal{K}$ can be written in the form

$$
\begin{aligned}
\left\{g^{i}(\boldsymbol{\theta},\right. & \left.X), g^{j}\left(\boldsymbol{\theta}^{\prime}, Y\right)\right\}\left.\right|_{\mathcal{K}} \\
= & \left\{\varphi^{i}(\boldsymbol{\theta}, X), \varphi^{j}\left(\boldsymbol{\theta}^{\prime}, Y\right)\right\}\left|\mathcal{K}-\left\{\varphi^{i}(\boldsymbol{\theta}, X), U^{\lambda}(Y)\right\}\right|_{\mathcal{K}} \Phi_{U^{\lambda}}^{j}\left(\frac{\mathbf{S}(Y)}{\epsilon}+\boldsymbol{\theta}^{\prime}, \mathbf{U}(Y)\right) \\
& -\left.\Phi_{U^{\nu}}^{i}\left(\frac{\mathbf{S}(X)}{\epsilon}+\boldsymbol{\theta}, \mathbf{U}(X)\right)\left\{U^{\nu}(X), \varphi^{j}\left(\boldsymbol{\theta}^{\prime}, Y\right)\right\}\right|_{\mathcal{K}} \\
& +\left.\Phi_{U^{\nu}}^{i}\left(\frac{\mathbf{S}(X)}{\epsilon}+\boldsymbol{\theta}, \mathbf{U}(X)\right)\left\{U^{\nu}(X), U^{\lambda}(Y)\right\}\right|_{\mathcal{K}} \Phi_{U^{\lambda}}^{j}\left(\frac{\mathbf{S}(Y)}{\epsilon}+\boldsymbol{\theta}^{\prime}, \mathbf{U}(Y)\right) \\
& -\left.\frac{1}{2 \epsilon} \int\left\{\varphi^{i}(\boldsymbol{\theta}, X), k^{\beta}(W)\right\}\right|_{\mathcal{K}} \operatorname{sgn}(Y-W) \mathrm{d} W \Phi_{\theta^{\prime \beta}}^{j}\left(\frac{\mathbf{S}(Y)}{\epsilon}+\boldsymbol{\theta}^{\prime}, \mathbf{U}(Y)\right) \\
& -\left.\frac{1}{2 \epsilon} \Phi_{\theta^{\alpha}}^{i}\left(\frac{\mathbf{S}(X)}{\epsilon}+\boldsymbol{\theta}, \mathbf{U}(X)\right) \int \operatorname{sgn}(X-Z)\left\{k^{\alpha}(Z), \varphi^{j}\left(\boldsymbol{\theta}^{\prime}, Y\right)\right\}\right|_{\mathcal{K}} \mathrm{d} Z \\
+ & \frac{1}{2 \epsilon} \Phi_{\theta^{\alpha}}^{i}\left(\frac{\mathbf{S}(X)}{\epsilon}+\boldsymbol{\theta}, \mathbf{U}(X)\right) \\
& \times\left.\int \operatorname{sgn}(X-Z)\left\{k^{\alpha}(Z), U^{\lambda}(Y)\right\}\right|_{\mathcal{K}} \mathrm{d} Z \Phi_{U^{\lambda}}^{j}\left(\frac{\mathbf{S}(Y)}{\epsilon}+\boldsymbol{\theta}^{\prime}, \mathbf{U}(Y)\right) \\
+ & \frac{1}{2 \epsilon} \Phi_{U^{\nu}}^{i}\left(\frac{\mathbf{S}(X)}{\epsilon}+\boldsymbol{\theta}, \mathbf{U}(X)\right) \\
& \times \int\left\{U^{\nu}(X), k^{\beta}(W)\right\} \mid \mathcal{K} \operatorname{sgn}(Y-W) \mathrm{d} W \Phi_{\theta^{\prime \beta}}^{j}\left(\frac{\mathbf{S}(Y)}{\epsilon}+\boldsymbol{\theta}^{\prime}, \mathbf{U}(Y)\right) \\
+ & \frac{1}{4 \epsilon^{2}} \Phi_{\theta^{\alpha}}^{i}\left(\frac{\mathbf{S}(X)}{\epsilon}+\boldsymbol{\theta}, \mathbf{U}(X)\right) \\
& \times\left.\int \operatorname{sgn}(X-Z)\left\{k^{\alpha}(Z), k^{\beta}(W)\right\}\right|_{\mathcal{K}} \operatorname{sgn}(Y-W) \mathrm{d} Z \mathrm{~d} W \Phi_{\theta^{\prime \beta}}^{j}\left(\frac{\mathbf{S}(Y)}{\epsilon}+\boldsymbol{\theta}^{\prime}, \mathbf{U}(Y)\right) .
\end{aligned}
$$

For the purposes of this chapter, we need only "approximate" restriction of the bracket (2.27) on the submanifold $\mathcal{K}$. Specifically, we will need to prove the existence of only the first term $\beta_{i(1)}^{\mu}(\boldsymbol{\theta}, X, Y)$ in the expansion $(2.53)$ having the form

$$
\beta_{i(1)}^{\mu}(\boldsymbol{\theta}, X, Y)=\beta_{i(1), 1}^{\mu}(\boldsymbol{\theta}, \mathbf{U}(X)) \delta^{\prime}(X-Y)+\beta_{i(1), 0 \lambda}^{\mu}(\boldsymbol{\theta}, \mathbf{U}(X)) U_{X}^{\lambda} \delta(X-Y),
$$

which is equivalent to the approximation

$$
B_{i[\mathbf{q}](1)}(\boldsymbol{\theta}, X)=\beta_{i(1), 1}^{\mu}(\boldsymbol{\theta}, \mathbf{U}(X)) q_{\mu X}(X)+\beta_{i(1), 0 \lambda}^{\mu}(\boldsymbol{\theta}, \mathbf{U}(X)) U_{X}^{\lambda} q_{\mu}(X)
$$

for the functions $B_{i[\mathbf{q}]}(\boldsymbol{\theta}, X)$.

To find it we need only the leading term of the convolution of the Poisson brackets of constraints on $\mathcal{K}$ with $B_{j[\mathbf{q}](1)}\left(\mathbf{S}(Y) / \epsilon+\boldsymbol{\theta}^{\prime}, Y\right)$ and the first (in $\left.\epsilon\right)$ non-vanishing term of the brackets of constraints and the functional $J_{[\mathbf{q}]}$.

Note that according to the relations (2.43), (2.44), (2.47), and (2.48) all the terms of the bracket (2.54) behave as the values of the order of at most $O(1)$ for $\epsilon \rightarrow 0$ in the convolution with $B_{j[\mathbf{q}](1)}\left(\mathbf{S}(Y) / \epsilon+\boldsymbol{\theta}^{\prime}, Y\right)$.

We impose additional conditions

$$
\int_{0}^{2 \pi} \cdots \int_{0}^{2 \pi} \Phi_{\theta^{\alpha}}^{j}(\boldsymbol{\theta}, \mathbf{U}(X)) \beta_{j(1)}^{\mu}(\boldsymbol{\theta}, X, Y) \frac{\mathrm{d}^{m} \theta}{(2 \pi)^{m}} \equiv 0, \quad \alpha=1, \ldots, m
$$


or equivalently

$$
\int_{0}^{2 \pi} \cdots \int_{0}^{2 \pi} \Phi_{\theta^{\alpha}}^{j}(\boldsymbol{\theta}, \mathbf{U}(X)) B_{j[\mathbf{q}](1)}(\boldsymbol{\theta}, X) \frac{\mathrm{d}^{m} \theta}{(2 \pi)^{m}} \equiv 0, \quad \alpha=1, \ldots, m,
$$

to be confirmed aposteriori for the functions $\beta_{j(1)}^{\mu}(\boldsymbol{\theta}, X, Y)$ and $B_{j[\mathbf{q}](1)}(\boldsymbol{\theta}, X)$. The Poisson brackets of constraints $(2.54)$ on $\mathcal{K}$ can then be reduced to the form

$$
\begin{aligned}
\left.\left.\left\{g^{i}(\boldsymbol{\theta}, X), g^{j}\left(\boldsymbol{\theta}^{\prime}, Y\right)\right\}\right|_{\mathcal{K}} \rightarrow\left\{g^{i}(\boldsymbol{\theta}, X), g^{j}\left(\boldsymbol{\theta}^{\prime}, Y\right)\right\}\right|_{\mathcal{K}} ^{\text {eff }} \\
=\left.\left\{\varphi^{i}(\boldsymbol{\theta}, X), \varphi^{j}\left(\boldsymbol{\theta}^{\prime}, Y\right)\right\}\right|_{\mathcal{K}}-\left.\left\{\varphi^{i}(\boldsymbol{\theta}, X), U^{\lambda}(Y)\right\}\right|_{\mathcal{K}} \Phi_{U^{\lambda}}^{j}\left(\frac{\mathbf{S}(Y)}{\epsilon}+\boldsymbol{\theta}^{\prime}, \mathbf{U}(Y)\right) \\
-\left.\Phi_{U^{\nu}}^{i}\left(\frac{\mathbf{S}(X)}{\epsilon}+\boldsymbol{\theta}, \mathbf{U}(X)\right)\left\{U^{\nu}(X), \varphi^{j}\left(\boldsymbol{\theta}^{\prime}, Y\right)\right\}\right|_{\mathcal{K}} \\
+\left.\Phi_{U^{\nu}}^{i}\left(\frac{\mathbf{S}(X)}{\epsilon}+\boldsymbol{\theta}, \mathbf{U}(X)\right)\left\{U^{\nu}(X), U^{\lambda}(Y)\right\}\right|_{\mathcal{K}} \Phi_{U^{\lambda}}^{j}\left(\frac{\mathbf{S}(Y)}{\epsilon}+\boldsymbol{\theta}^{\prime}, \mathbf{U}(Y)\right) \\
-\left.\frac{1}{2 \epsilon} \Phi_{\theta^{\alpha}}^{i}\left(\frac{\mathbf{S}(X)}{\epsilon}+\boldsymbol{\theta}, \mathbf{U}(X)\right) \int \operatorname{sgn}(X-Z)\left\{k^{\alpha}(Z), \varphi^{j}\left(\boldsymbol{\theta}^{\prime}, Y\right)\right\}\right|_{\mathcal{K}} \mathrm{d} Z \\
+\left.\frac{1}{2 \epsilon} \Phi_{\theta^{\alpha}}^{i}\left(\frac{\mathbf{S}(X)}{\epsilon}+\boldsymbol{\theta}, \mathbf{U}(X)\right) \int \operatorname{sgn}(X-Z)\left\{k^{\alpha}(Z), U^{\lambda}(Y)\right\}\right|_{\mathcal{K}} \mathrm{d} Z \\
\quad \times \Phi_{U^{\lambda}}^{j}\left(\frac{\mathbf{S}(Y)}{\epsilon}+\boldsymbol{\theta}^{\prime}, \mathbf{U}(Y)\right)
\end{aligned}
$$

in finding $B_{i[\mathbf{q}](1)}(\boldsymbol{\theta}, X)$.

To find the leading term of the convolution of the Poisson brackets of constraints on $\mathcal{K}$ and $B_{j[\mathbf{q}](1)}\left(\mathbf{S}(Y) / \epsilon+\boldsymbol{\theta}^{\prime}, Y\right)$ we can simplify further the relation (2.59). Namely, taking into account relations $(2.41),(2.47)$ we can omit in the leading order the second and the last terms of (2.59) in the convolution with $B_{j[\mathbf{q}](1)}\left(\mathbf{S}(Y) / \epsilon+\boldsymbol{\theta}^{\prime}, Y\right)$. By (2.42) and (2.58), we can omit in the leading order also the third term of (2.59) in the convolution with $B_{j[\mathbf{q}](1)}\left(\mathbf{S}(Y) / \epsilon+\boldsymbol{\theta}^{\prime}, Y\right)$. The fourth term of (2.59), obviously, is of the order $O(\epsilon)$ and also can be omitted in the leading order.

As a result, the principal term of the convolution of (2.54) with the functions $\epsilon B_{j[\mathbf{q}](1)}\left(\mathbf{S}(Y) / \epsilon+\boldsymbol{\theta}^{\prime}, Y\right)$ can be written in the form

$$
\begin{aligned}
& {\left[\left.\int\left\{g^{i}(\boldsymbol{\theta}, X), g^{j}\left(\boldsymbol{\theta}^{\prime}, Y\right)\right\}\right|_{\mathcal{K}} \epsilon B_{j[\mathbf{q}](1)}\left(\frac{\mathbf{S}(Y)}{\epsilon}+\boldsymbol{\theta}^{\prime}, Y\right) \frac{\mathrm{d}^{m} \theta^{\prime}}{(2 \pi)^{m}} \mathrm{~d} Y\right]_{[1]}} \\
& \equiv \sum_{s \geq 0} B_{(s)}^{i j}\left(\boldsymbol{\Phi}\left(\frac{\mathbf{S}(X)}{\epsilon}+\boldsymbol{\theta}, \mathbf{U}(X)\right), k^{\gamma}(X) \mathbf{\Phi}_{\theta^{\gamma}}\left(\frac{\mathbf{S}(X)}{\epsilon}+\boldsymbol{\theta}, \mathbf{U}(X)\right), \ldots\right) \\
& \times k^{\alpha_{1}}(X) \cdots k^{\alpha_{s}}(X) B_{j[\mathbf{q}](1) \theta^{\alpha_{1} \ldots \theta^{\alpha_{s}}}}\left(\frac{\mathbf{S}(X)}{\epsilon}+\boldsymbol{\theta}, X\right) \\
& -\frac{1}{2} \Phi_{\theta^{\alpha}}^{i}\left(\frac{\mathbf{S}(X)}{\epsilon}+\boldsymbol{\theta}, \mathbf{U}(X)\right) \int \operatorname{sgn}(X-Z)
\end{aligned}
$$

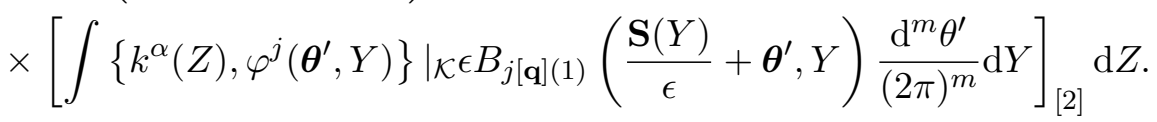

Let us remind here that the indexes [1], [2] mean as before the terms of the corresponding graded expansion on $\mathcal{K}$ having degree 1 and 2 respectively.

We have also

$$
\left.\left\{g^{i}(\boldsymbol{\theta}, X), J_{[\mathbf{q}]}\right\}\right|_{\mathcal{K}[1]}=\left.\left\{\varphi^{i}(\boldsymbol{\theta}, X), J_{[\mathbf{q}]}\right\}\right|_{\mathcal{K}[1]}-\left.\Phi_{U^{\nu}}^{i}\left(\frac{\mathbf{S}(X)}{\epsilon}+\boldsymbol{\theta}, \mathbf{U}(X)\right)\left\{U^{\nu}(X), J_{[\mathbf{q}]}\right\}\right|_{\mathcal{K}[1]}
$$




$$
-\left.\frac{1}{2} \Phi_{\theta^{\alpha}}^{i}\left(\frac{\mathbf{S}(X)}{\epsilon}+\boldsymbol{\theta}, \mathbf{U}(X)\right) \int \operatorname{sgn}(X-Z)\left\{k^{\alpha}(Z), J_{[\mathbf{q}]}\right\}\right|_{\mathcal{K}[2]} \mathrm{d} Z
$$

Let us prove here the following lemma which allows to investigate the further construction of the functions $B_{i[\mathbf{q}](1)}(\boldsymbol{\theta}, X)$.

Lemma 2.5. Let the functions $B_{i[\mathbf{q}](1)}(\boldsymbol{\theta}, X)$ satisfy the conditions (2.58), i.e.

$$
\int_{0}^{2 \pi} \cdots \int_{0}^{2 \pi} \Phi_{\theta^{\alpha}}^{j}(\boldsymbol{\theta}, \mathbf{U}(X)) B_{j[\mathbf{q}](1)}(\boldsymbol{\theta}, X) \frac{\mathrm{d}^{m} \theta}{(2 \pi)^{m}} \equiv 0
$$

and the relation

$$
\hat{B}_{[0]}^{i j}(X) B_{j[\mathbf{q}](1)}(\boldsymbol{\theta}, X)+A_{[1][\mathbf{q}]}^{i}(\boldsymbol{\theta}, X)=0,
$$

where

$$
\begin{aligned}
& \hat{B}_{[0]}^{i j}(X) \\
& \quad=\sum_{s \geq 0} B_{(s)}^{i j}\left(\boldsymbol{\Phi}(\boldsymbol{\theta}, \mathbf{U}(X)), k^{\alpha}(X) \boldsymbol{\Phi}_{\theta^{\alpha}}(\boldsymbol{\theta}, \mathbf{U}(X)), \ldots\right) k^{\alpha_{1}}(X) \cdots k^{\alpha_{s}}(X) \frac{\partial^{s}}{\partial \theta^{\alpha_{1}} \cdots \partial \theta^{\alpha_{s}}} \\
& A_{[1][\mathbf{q}]}^{i}\left(\frac{\mathbf{S}(X)}{\epsilon}+\boldsymbol{\theta}, X\right) \\
& \quad=\left.\left\{\varphi^{i}(\boldsymbol{\theta}, X), J_{[\mathbf{q}]}\right\}\right|_{\mathcal{K}[1]}-\left.\Phi_{U^{\nu}}^{i}\left(\frac{\mathbf{S}(X)}{\epsilon}+\boldsymbol{\theta}, \mathbf{U}(X)\right)\left\{U^{\nu}(X), J_{[\mathbf{q}]}\right\}\right|_{\mathcal{K}[1]} .
\end{aligned}
$$

Then the functions $B_{i[\mathbf{q}](1)}(\boldsymbol{\theta}, X)$ satisfy the relation

$$
\begin{aligned}
& {\left[\left.\int\left\{g^{i}(\boldsymbol{\theta}, X), g^{j}\left(\boldsymbol{\theta}^{\prime}, Z\right)\right\}\right|_{\mathcal{K}} \in B_{j[\mathbf{q}](1)}\left(\frac{\mathbf{S}(Z)}{\epsilon}+\boldsymbol{\theta}^{\prime}, Z\right) \frac{\mathrm{d}^{m} \theta^{\prime}}{(2 \pi)^{m}} \mathrm{~d} Z\right]_{[1]}} \\
& \quad+\left.\left\{g^{i}(\boldsymbol{\theta}, X), J_{[\mathbf{q}]}\right\}\right|_{\mathcal{K}[1]}=0 .
\end{aligned}
$$

Proof. The fulfillment of condition (2.58) allows to reduce the expression

$$
\left.\int\left\{g^{i}(\boldsymbol{\theta}, X), g^{j}\left(\boldsymbol{\theta}^{\prime}, Z\right)\right\}\right|_{\mathcal{K}} \epsilon B_{j[\mathbf{q}](1)}\left(\frac{\mathbf{S}(Z)}{\epsilon}+\boldsymbol{\theta}^{\prime}, Z\right) \frac{\mathrm{d}^{m} \theta^{\prime}}{(2 \pi)^{m}} \mathrm{~d} Z
$$

according to formula (2.60) up to the terms $O\left(\epsilon^{2}\right)$. From (2.62) we can get then

$$
\begin{aligned}
&\left.\int\left\{g^{i}(\boldsymbol{\theta}, X), g^{j}\left(\boldsymbol{\theta}^{\prime}, Z\right)\right\}\right|_{\mathcal{K}} \epsilon B_{j[\mathbf{q}](1)}\left(\frac{\mathbf{S}(Z)}{\epsilon}+\boldsymbol{\theta}^{\prime}, Z\right) \frac{\mathrm{d}^{m} \theta^{\prime}}{(2 \pi)^{m}} \mathrm{~d} Z+\left.\left\{g^{i}(\boldsymbol{\theta}, X), J_{[\mathbf{q}]}\right\}\right|_{\mathcal{K}} \\
&=- \frac{\epsilon}{2} \Phi_{\theta^{\alpha}}^{i}\left(\frac{\mathbf{S}(X)}{\epsilon}+\boldsymbol{\theta}, \mathbf{U}(X)\right) \int \operatorname{sgn}(X-Z) \\
& \times {\left[\left.\int\left\{k^{\alpha}(Z), \varphi^{j}\left(\boldsymbol{\theta}^{\prime}, W\right)\right\}\right|_{\mathcal{K}} \epsilon B_{j[\mathbf{q}](1)}\left(\frac{\mathbf{S}(W)}{\epsilon}+\boldsymbol{\theta}^{\prime}, W\right) \frac{\mathrm{d}^{m} \theta^{\prime}}{(2 \pi)^{m}} \mathrm{~d} W\right]_{[2]} \mathrm{d} Z } \\
&-\left.\frac{\epsilon}{2} \Phi_{\theta^{\alpha}}^{i}\left(\frac{\mathbf{S}(X)}{\epsilon}+\boldsymbol{\theta}, \mathbf{U}(X)\right) \int \operatorname{sgn}(X-Z)\left\{k^{\alpha}(Z), J_{[\mathbf{q}]}\right\}\right|_{\mathcal{K}[2]} \mathrm{d} Z+O\left(\epsilon^{2}\right) .
\end{aligned}
$$

The proof of the Lemma follows then from the orthogonality conditions of the left-hand part of (2.66) to the variational derivatives

$$
\left.\frac{\delta k^{\beta}\left(\mathbf{J}\left(X^{\prime}\right)\right)}{\delta \varphi^{i}(\boldsymbol{\theta}, X)}\right|_{\mathcal{K}}=\left.\frac{\partial k^{\beta}}{\partial U^{\lambda}}\left(\mathbf{J}\left(X^{\prime}\right)\right) \frac{\delta J^{\lambda}\left(X^{\prime}\right)}{\delta \varphi^{i}(\boldsymbol{\theta}, X)}\right|_{\mathcal{K}}, \quad \beta=1, \ldots, m
$$


according to $(2.33)$ in the order $\epsilon^{2}$ on $\mathcal{K}$. Here we use the functions $k^{\beta}(\mathbf{U})$ to define the functionals $k^{\beta}\left(\mathbf{J}\left(X^{\prime}\right)\right)$. Let us remind also that the functionals $\mathbf{U}(X)$ and $\mathbf{J}(X)$ are connected by the transformations (2.30) and (2.31).

Indeed, the right-hand part of (2.66) has the form

$$
\frac{\epsilon}{2} \Phi_{\theta^{\alpha}}^{i}\left(\frac{\mathbf{S}(X)}{\epsilon}+\boldsymbol{\theta}, \mathbf{U}(X)\right) \int \operatorname{sgn}(X-Z) Q_{[\mathbf{q}]}^{\alpha}(Z) \mathrm{d} Z+O\left(\epsilon^{2}\right)
$$

with some functions $Q_{[\mathbf{q}]}^{\alpha}(Z)$.

The convolution with the quantities (2.67) can be represented as the action of the operator

$$
\begin{aligned}
\int_{0}^{2 \pi} & \cdots \int_{0}^{2 \pi} \frac{\mathrm{d}^{m} \theta}{(2 \pi)^{m}} \frac{\partial k^{\beta}}{\partial U^{\lambda}}\left(\mathbf{J}\left(X^{\prime}\right)\right) \\
& \times \sum_{l \geq 0} \Pi_{i(l)}^{\lambda}\left(\boldsymbol{\Phi}\left(\frac{\mathbf{S}\left(X^{\prime}\right)}{\epsilon}+\boldsymbol{\theta}, \mathbf{U}\left(X^{\prime}\right)\right), \epsilon \frac{\partial}{\partial X^{\prime}} \boldsymbol{\Phi}\left(\frac{\mathbf{S}\left(X^{\prime}\right)}{\epsilon}+\boldsymbol{\theta}, \mathbf{U}\left(X^{\prime}\right)\right), \ldots\right) \epsilon^{l} \frac{\mathrm{d}^{l}}{\mathrm{~d} X^{\prime l}}
\end{aligned}
$$

on the functions of $\mathbf{S}\left(X^{\prime}\right) / \epsilon+\boldsymbol{\theta}$ and $X^{\prime}$.

Expanding the action of the operator (2.69) in powers of $\epsilon$ we can write it's main part in the form

$$
\begin{aligned}
\int_{0}^{2 \pi} & \cdots \int_{0}^{2 \pi} \frac{\mathrm{d}^{m} \theta}{(2 \pi)^{m}} \frac{\partial k^{\beta}}{\partial U^{\lambda}}\left(\mathbf{U}\left(X^{\prime}\right)\right) \\
& \times \sum_{l \geq 0} \Pi_{i(l)}^{\lambda}\left(\boldsymbol{\Phi}(\ldots), k^{\alpha} \boldsymbol{\Phi}_{\theta^{\alpha}}(\ldots), \ldots\right) k^{\alpha_{1}}\left(X^{\prime}\right) \cdots k^{\alpha_{l}}\left(X^{\prime}\right) \frac{\partial^{l}}{\partial \theta^{\alpha_{1}} \cdots \partial \theta^{\alpha_{l}}} .
\end{aligned}
$$

We can see then after the integration by parts that the main part of the operator (2.69) reduces to the convolution (w.r.t. $\boldsymbol{\theta}$ ) with the vectors

$$
\frac{\partial k^{\beta}}{\partial U^{\lambda}}\left(\mathbf{U}\left(X^{\prime}\right)\right) \zeta_{i\left[\mathbf{U}\left(X^{\prime}\right)\right]}^{(\lambda)}\left(\frac{\mathbf{S}\left(X^{\prime}\right)}{\epsilon}+\boldsymbol{\theta}\right)
$$

which are identically equal to zero according to Lemma 2.1 .

We can conclude, therefore, that terms of the order of $O\left(\epsilon^{2}\right)$ in the right-hand part of (2.66) do not play any role in the convolution with the values (2.67) in the order $\epsilon^{2}$.

Let us consider now the action of the operator (2.69) on the terms of the order of $\epsilon$ in the expression (2.68). We can see that the terms in which the operators $\mathrm{d}^{s} / \mathrm{d} X^{\prime s}$ apply only to the functions $\boldsymbol{\Phi}_{\theta^{\alpha}}\left(\mathbf{S}\left(X^{\prime}\right) / \epsilon+\boldsymbol{\theta}, \mathbf{U}\left(X^{\prime}\right)\right)$ yield the quantity

$$
\left.\frac{\epsilon}{2} \frac{\partial k^{\beta}}{\partial U^{\lambda}}\left(\mathbf{J}\left(X^{\prime}\right)\right) \int_{0}^{2 \pi} \cdots \int_{0}^{2 \pi} \frac{\partial}{\partial \theta^{\alpha}} P^{\lambda}\left(\boldsymbol{\varphi}, \epsilon \boldsymbol{\varphi}_{X^{\prime}}, \ldots\right)\right|_{\mathcal{K}} \frac{\mathrm{d}^{m} \theta}{(2 \pi)^{m}} \int \operatorname{sgn}\left(X^{\prime}-Z\right) Q_{[\mathbf{q}]}^{\alpha}(Z) \mathrm{d} Z
$$

identically equal to zero.

The terms of the order of $\epsilon^{2}$ occur at the same time with a single differentiation of the function $\operatorname{sgn}\left(X^{\prime}-Z\right)$ and application of the remaining derivations $\partial / \partial X^{\prime}$ to $\boldsymbol{\Phi}_{\theta^{\alpha}}\left(\mathbf{S}\left(X^{\prime}\right) / \epsilon+\boldsymbol{\theta}, \mathbf{U}\left(X^{\prime}\right)\right)$ in the leading order. In the required approximation the functionals $\mathbf{J}(X)$ should be also replaced by $\mathbf{U}(X)$ in the argument of $\partial k^{\beta} / \partial U^{\lambda}$.

The corresponding values are then equal to

$$
\begin{aligned}
& \epsilon^{2} \frac{\partial k^{\beta}}{\partial U^{\lambda}}\left(\mathbf{U}\left(X^{\prime}\right)\right) \\
& \quad \times \int_{0}^{2 \pi} \cdots \int_{0}^{2 \pi} \sum_{l \geq 1} \Pi_{i(l)}^{\lambda}\left(\boldsymbol{\Phi}, k^{\gamma} \boldsymbol{\Phi}_{\theta^{\gamma}}, \ldots\right) l k^{\alpha_{1}} \cdots k^{\alpha_{l-1}} \Phi_{\theta^{\alpha_{1} \ldots \theta^{\alpha} l-1} \theta^{\alpha}}^{i} \frac{\mathrm{d}^{m} \theta}{(2 \pi)^{m}} Q_{[\mathbf{q}]}^{\alpha}\left(X^{\prime}\right) .
\end{aligned}
$$


Using again the relations (2.13), we obtain that the orthogonality of the right-hand part of (2.66) to the values (2.67) in the order $\epsilon^{2}$ is equivalent to the relations $Q_{[\mathbf{q}]}^{\beta}\left(X^{\prime}\right) \equiv 0$. Given that the main part of (2.68) represents the difference between the left-hand sides of (2.65) and (2.62) we obtain the assertion of the lemma.

For a regular Hamiltonian family $\Lambda$ and a complete Hamiltonian set of integrals $\left(I^{1}, \ldots, I^{N}\right)$ we can also prove the following lemma.

Lemma 2.6. Let the functions $B_{j[\mathbf{q}](1)}(\boldsymbol{\theta}, X)$ satisfy conditions (2.62). Then the functions $B_{j[\mathbf{q}](1)}(\boldsymbol{\theta}, X)$ automatically satisfy $(2.58)$.

Proof. We first prove the following statement.

The values $\zeta_{i[\mathbf{U}(X)]}^{(\nu)}(\boldsymbol{\theta})$ are orthogonal (for any $\left.X\right)$ to the values $A_{[1][\mathbf{q}]}^{i}(\boldsymbol{\theta}, X)$, i.e.

$$
\int_{0}^{2 \pi} \cdots \int_{0}^{2 \pi} \zeta_{i[\mathbf{U}(X)]}^{(\nu)}(\boldsymbol{\theta}) A_{[1][\mathbf{q}]}^{i}(\boldsymbol{\theta}, X) \frac{\mathrm{d}^{m} \theta}{(2 \pi)^{m}} \equiv 0 .
$$

Indeed, as we know, the values $\left.\left\{g^{i}(\boldsymbol{\theta}, X), J_{[\mathbf{q}]}\right\}\right|_{\mathcal{K}}$ identically vanish under action of the operator (2.34) (with the replacement of $Z$ to $X)$. In the leading order $(\sim \epsilon)$ it's action is given by the convolution w.r.t. $\boldsymbol{\theta}$ of the values $\left.\left\{g^{i}(\boldsymbol{\theta}, X), J_{[\mathbf{q}]}\right\}\right|_{\mathcal{K}[1]}$, imposed by (2.61), with the values $\zeta_{i[\mathbf{U}(X)]}^{(\nu)}(\mathbf{S}(X) / \epsilon+\boldsymbol{\theta})$. We know also that the values $\zeta_{i[\mathbf{U}(X)]}^{(\nu)}(\mathbf{S}(X) / \epsilon+\boldsymbol{\theta})$ are automatically orthogonal to the functions $\Phi_{\theta^{\alpha}}^{i}(\mathbf{S}(X) / \epsilon+\boldsymbol{\theta}, \mathbf{U}(X))$, so we get the relation (2.70).

Thus, the implementation of (2.62) implies the conditions

$$
\int_{0}^{2 \pi} \cdots \int_{0}^{2 \pi} \zeta_{i[\mathbf{U}(X)]}^{(\nu)}(\boldsymbol{\theta}) \hat{B}_{[0]}^{i j}(X) B_{j[\mathbf{q}](1)}(\boldsymbol{\theta}, X) \frac{\mathrm{d}^{m} \theta}{(2 \pi)^{m}}=0,
$$

which is equivalent to

$$
\omega^{\alpha \nu}(\mathbf{U}(X)) \int_{0}^{2 \pi} \cdots \int_{0}^{2 \pi} \Phi_{\theta^{\alpha}}^{j}(\boldsymbol{\theta}, \mathbf{U}(X)) B_{j[\mathbf{q}](1)}(\boldsymbol{\theta}, X) \frac{\mathrm{d}^{m} \theta}{(2 \pi)^{m}}=0
$$

view the skew-symmetry of $\hat{B}_{[0]}^{i j}(X)$.

From condition (2) of the definition of a complete Hamiltonian family of commuting functionals we immediately obtain now conditions (2.58).

It is not difficult to see that from the relation (2.70) follow also the conditions

$$
\int_{0}^{2 \pi} \cdots \int_{0}^{2 \pi} \kappa_{i[\mathbf{U}(X)]}^{(q)}(\boldsymbol{\theta}) A_{[1][\mathbf{q}]}^{i}(\boldsymbol{\theta}, X) \frac{\mathrm{d}^{m} \theta}{(2 \pi)^{m}} \equiv 0
$$

by Proposition 2.1 .

We come, therefore, to investigation of system (2.62) to construct an "approximate" restriction of the Poisson bracket (2.27) on the submanifold $\mathcal{K}$. In the remaining part of the article the investigation of system (2.62) will play the basic role for the justification of the main results.

In what follows we consider separately the single-phase $(m=1)$ and the multiphase $(m \geq 2)$ cases.

The following lemma can be formulated for the single-phase case $m=1$.

Lemma 2.7. Let $\Lambda$ be a regular Hamiltonian family of single-phase solutions of (2.3) and $\left(I^{1}, \ldots, I^{N}\right)$ be a complete Hamiltonian set of the first integrals of the form (2.18). Then the functions $B_{j[\mathbf{q}](1)}(\boldsymbol{\theta}, X)$ can be found from system (2.62) and can be written in the form (2.56) with smooth dependence on parameters $\mathbf{U}(X)$. 
Proof. System (2.62) in the single-phase case is a system of ordinary differential equations in $\theta$ with skew-symmetric operator $\hat{B}_{[0]}^{i j}(X)$. It is easy to see also that the right-hand side of system (2.62) has the form

$$
\xi^{i \mu}(\theta, \mathbf{U}(X)) q_{\mu, X}(X)+\eta_{\lambda}^{i \mu}(\theta, \mathbf{U}(X)) U_{X}^{\lambda} q_{\mu}(X)
$$

with periodic in $\theta$ functions $\xi^{i \mu}(\theta, \mathbf{U}(X)), \eta_{\lambda}^{i \mu}(\theta, \mathbf{U}(X))$.

The orthogonality conditions (2.71) imply the orthogonality of both the sets of functions $\xi^{i \mu}(\theta, \mathbf{U}(X))$ and $\eta_{\lambda}^{i \mu}(\theta, \mathbf{U}(X))$ to the functions $\kappa_{i[\mathbf{U}(X)]}^{(q)}(\theta)$, such that system (2.62) can be split into independent inhomogeneous systems

$$
\begin{aligned}
& \hat{B}_{[0]}^{i j}(X) \beta_{j(1), 1}^{\mu}(\theta, \mathbf{U}(X))=\xi^{i \mu}(\theta, \mathbf{U}(X)), \\
& \hat{B}_{[0]}^{i j}(X) \beta_{j(1), 0 \lambda}^{\mu}(\theta, \mathbf{U}(X))=\eta_{\lambda}^{i \mu}(\theta, \mathbf{U}(X))
\end{aligned}
$$

defining functions (2.55).

Both the systems (2.72), (2.73) are systems of ordinary linear differential equations with periodic coefficients and the skew-symmetric operator $\hat{B}_{[0]}^{i j}(X)$. The zero modes of the operator $\hat{B}_{[0]}^{i j}(X)$ are given by the variational derivatives of annihilators of the bracket (2.16) represented as functions of $\theta$ on the manifold of single-phase solutions and are orthogonal to the right-hand parts of (2.72) and (2.73) according to (2.26) and (2.71). Eigenfunctions of $\hat{B}_{[0]}^{i j}(X)$ form a basis in the space of $2 \pi$-periodic functions $\varphi(\theta)$. Besides that, the nonzero eigenvalues of $\hat{B}_{[0]}^{i j}(X)$ are separated from zero in this case. Thus, the $2 \pi$-periodic functions $\beta_{j(1), 1}^{\mu}(\theta, \mathbf{U}(X))$, $\beta_{j(1), 0 \lambda}^{\mu}(\theta, \mathbf{U}(X))$ can be found from systems (2.72), (2.73) up to the variational derivatives of the annihilators of the bracket (2.16). If we impose additional conditions of orthogonality of $\beta_{j(1)}^{\mu}(\theta, X, Y)$ to the variational derivatives of the annihilators of the bracket $(2.16)$ on the manifold of single-phase solutions we can suggest a unique procedure of construction of the functions $\beta_{j(1)}^{\mu}(\theta, X, Y)$. The functions $\beta_{j(1)}^{\mu}(\theta, X, Y)$ satisfy $(2.52)$ in the order of $O(\epsilon)$ and conditions (2.57) and smoothly depend on the parameters $\mathbf{U}(X)$, which implies the required properties for the functions $B_{j[\mathbf{q}](1)}(\theta, X)$.

Let us formulate now the theorem that gives the justification of the Dubrovin-Novikov procedure for a regular Hamiltonian family of single-phase solutions of system (2.3).

Theorem 2.1. Let $\Lambda$ be a regular Hamiltonian family of single-phase solutions of system (2.3). Let $\left(I^{1}, \ldots, I^{N}\right)$ be a complete Hamiltonian set of commuting first integrals of system (2.3) on $\Lambda$ having the form (2.18). Then the Dubrovin-Novikov procedure gives a Poisson bracket of hydrodynamic type on the space of parameters $\left\{U^{\nu}(X), \nu=1, \ldots, N\right\}$, where $U^{\nu} \equiv\left\langle P^{\nu}\right\rangle$.

Proof. The antisymmetry of the Dubrovin-Novikov bracket follows immediately from the antisymmetry of the original bracket. Let us prove the existence of the Jacobi identity under the conditions of the theorem.

Consider the Poisson bracket (2.27) and the functionals

$$
\tilde{J}_{(1)}^{\nu}(X)=J^{\nu}(X)+\epsilon \iint_{0}^{2 \pi} g^{j}(\theta, Z) \beta_{j(1)}^{\nu}\left(\frac{S(Z)}{\epsilon}+\theta, Z, X\right) \frac{\mathrm{d} \theta}{2 \pi} \mathrm{d} Z
$$

in the neighborhood of the submanifold $\mathcal{K}$. (Note that the functions $\beta_{j(1)}^{\nu}$ depending on the values $\mathbf{U}(X)$ on $\mathcal{K}$, can be considered as functionals in the neighborhood of $\mathcal{K}$ by the definition of the functionals $\mathbf{U}(X)$ ).

The pairwise brackets of the functionals $\tilde{J}_{(1)}^{\nu}(X), \tilde{J}_{(1)}^{\mu}(Y)$ can be written as

$$
\left\{\tilde{J}_{(1)}^{\nu}(X), \tilde{J}_{(1)}^{\mu}(Y)\right\}=\left\{J^{\nu}(X), J^{\mu}(Y)\right\}
$$




$$
\begin{aligned}
& +\epsilon \iint_{0}^{2 \pi}\left\{\beta_{i(1)}^{\nu}\left(\frac{S(Z)}{\epsilon}+\theta, Z, X\right) g^{i}(\theta, Z), J^{\mu}(Y)\right\} \frac{\mathrm{d} \theta}{2 \pi} \mathrm{d} Z \\
& +\epsilon \iint_{0}^{2 \pi}\left\{J^{\nu}(X), g^{j}(\theta, W) \beta_{j(1)}^{\mu}\left(\frac{S(W)}{\epsilon}+\theta, W, Y\right)\right\} \frac{\mathrm{d} \theta}{2 \pi} \mathrm{d} W \\
& +\epsilon^{2} \int\left\{\beta_{i(1)}^{\nu}\left(\frac{S(Z)}{\epsilon}+\theta, Z, X\right) g^{i}(\theta, Z), g^{j}\left(\theta^{\prime}, W\right) \beta_{j(1)}^{\mu}\left(\frac{S(W)}{\epsilon}+\theta^{\prime}, W, Y\right)\right\} \frac{\mathrm{d} \theta}{2 \pi} \frac{\mathrm{d} \theta^{\prime}}{2 \pi} \mathrm{d} Z \mathrm{~d} W .
\end{aligned}
$$

We note here that despite the presence of non-local terms in the brackets of constraints $\left\{g^{i}(\theta, Z), g^{j}\left(\theta^{\prime}, W\right)\right\}$, and $\left\{J^{\nu}(X), g^{j}(\theta, W)\right\},\left\{g^{i}(\theta, Z), J^{\mu}(Y)\right\}$, the bracket of the functionals $\left\{\tilde{J}_{(1)}^{\nu}(X), \tilde{J}_{(1)}^{\mu}(Y)\right\}$ on $\mathcal{K}$ has a purely local form because of the conditions (2.57). We can also see here that expression (2.74) is a regular at $\epsilon \rightarrow 0$ distribution after the restriction on $\mathcal{K}$.

From the form of the constraints and condition (2.57) it is easy to obtain that the pairwise Poisson brackets of the functionals $\tilde{J}_{(1)}^{\nu}(X), \tilde{J}_{(1)}^{\mu}(Y)$ on $\mathcal{K}$ can be written as

$$
\left.\left\{\tilde{J}_{(1)}^{\nu}(X), \tilde{J}_{(1)}^{\mu}(Y)\right\}\right|_{\mathcal{K}}=\sum_{l \geq 1} \epsilon^{l} \sum_{q=0}^{l} a_{[l-q]}^{\nu \mu}\left(\mathbf{U}, \mathbf{U}_{X}, \ldots\right) \delta^{(q)}(X-Y)
$$

where $a_{[l-q]}^{\nu \mu}$ are some functions of $\mathbf{U}, \mathbf{U}_{X}, \ldots$, polynomial in the derivatives and having degree $l-q$. By virtue of relations $(2.51)$ we can state also that the leading term in $\epsilon$ in the expression (2.75) is still the same Dubrovin-Novikov bracket on $\mathcal{K}$, since the corrections given by the last three terms of (2.74) are of the order of $O\left(\epsilon^{2}\right)$ on $\mathcal{K}$. We thus have

$$
\left.\left\{\tilde{J}_{(1)}^{\nu}(X), \tilde{J}_{(1)}^{\mu}(Y)\right\}\right|_{\mathcal{K}}=\epsilon\left\{U^{\nu}(X), U^{\mu}(Y)\right\}_{\mathrm{DN}}+\sum_{l \geq 2} \epsilon^{l} \sum_{q=0}^{l} a_{[l-q]}^{\nu \mu}\left(\mathbf{U}, \mathbf{U}_{X}, \ldots\right) \delta^{(q)}(X-Y)
$$

where

$$
\left\{U^{\nu}(X), U^{\mu}(Y)\right\}_{\mathrm{DN}}=\left\langle A_{1}^{\nu \mu}\right\rangle(\mathbf{U}(X)) \delta^{\prime}(X-Y)+\frac{\partial\left\langle Q^{\nu \mu}\right\rangle}{\partial U^{\gamma}} U_{X}^{\gamma} \delta(X-Y)
$$

is the Dubrovin-Novikov bracket on the space of fields $\mathbf{U}(X)$. Thus, we can write

$$
\begin{gathered}
\left.\frac{\delta}{\delta U^{\gamma}(W)}\left\{\tilde{J}_{(1)}^{\nu}(X), \tilde{J}_{(1)}^{\mu}(Y)\right\}\right|_{\mathcal{K}}=\epsilon \frac{\delta\left\{U^{\nu}(X), U^{\mu}(Y)\right\}_{\mathrm{DN}}}{\delta U^{\gamma}(W)} \\
\quad+\frac{\delta}{\delta U^{\gamma}(W)} \sum_{l \geq 2} \epsilon^{l} \sum_{q=0}^{l} a_{[l-q]}^{\nu \mu}\left(\mathbf{U}, \mathbf{U}_{X}, \ldots\right) \delta^{(q)}(X-Y)
\end{gathered}
$$

on the submanifold $\mathcal{K}$.

For further discussion it is convenient to introduce "regularized" functionals. Namely, for any smooth compactly supported vector-valued function $\mathbf{q}(X)=\left(q_{1}(X), \ldots, q_{N}(X)\right)$ we introduce the functionals

$$
J_{[\mathbf{q}]}=\int q_{\nu}(X) J^{\nu}(X) \mathrm{d} X, \quad \tilde{J}_{(1)[\mathbf{q}]}=\int q_{\nu}(X) \tilde{J}_{(1)}^{\nu}(X) \mathrm{d} X, \quad U_{[\mathbf{q}]}=\int q_{\nu}(X) U^{\nu}(X) \mathrm{d} X .
$$

We can write then

$$
\begin{aligned}
& \left\{\tilde{J}_{(1)[\mathbf{q}]}, \tilde{J}_{(1)[\mathbf{p}]}\right\}=\left\{J_{[\mathbf{q}]}, J_{[\mathbf{p}]}\right\}+\epsilon \int_{-\infty}^{+\infty} \int_{0}^{2 \pi}\left\{B_{i[\mathbf{q}](1)}\left(\frac{S(Z)}{\epsilon}+\theta, Z\right) g^{i}(\theta, Z), J_{[\mathbf{p}]}\right\} \frac{\mathrm{d} \theta}{2 \pi} \mathrm{d} Z \\
& +\epsilon \int_{-\infty}^{+\infty} \int_{0}^{2 \pi}\left\{J_{[\mathbf{q}]}, g^{j}(\theta, W) B_{j[\mathbf{p}](1)}\left(\frac{S(W)}{\epsilon}+\theta, W\right)\right\} \frac{\mathrm{d} \theta}{2 \pi} \mathrm{d} W
\end{aligned}
$$




$$
+\epsilon^{2} \int\left\{B_{i[\mathbf{q}](1)}\left(\frac{S(Z)}{\epsilon}+\theta, Z\right) g^{i}(\theta, Z), g^{j}\left(\theta^{\prime}, W\right) B_{j[\mathbf{p}](1)}\left(\frac{S(W)}{\epsilon}+\theta^{\prime}, W\right)\right\} \frac{\mathrm{d} \theta}{2 \pi} \frac{\mathrm{d} \theta^{\prime}}{2 \pi} \mathrm{d} Z \mathrm{~d} W
$$

We can see again that the last three terms of (2.77) give corrections of the order of $\epsilon^{2}$ on the submanifold $\mathcal{K}$.

For the expansion of the brackets (2.77) in the neighborhood of the submanifold $\mathcal{K}$ it is convenient to use the expressions

$$
\varphi^{i}(\theta, X)=g^{i}(\theta, X)+\Phi^{i}\left(\frac{S(X)}{\epsilon}+\theta, \mathbf{U}(X)\right)
$$

making it possible to easily expand any functionals of $\varphi(\theta, X)$ with respect to the values of $\mathbf{g}$. For example, using the expression $(2.20)$, we can write for the brackets $\left\{J_{[\mathbf{q}]}, J_{[\mathbf{p}]}\right\}$ in the neighborhood of $\mathcal{K}$

$$
\begin{aligned}
& \left\{J_{[\mathbf{q}]}, J_{[\mathbf{p}]}\right\}=\left.\left\{J_{[\mathbf{q}]}, J_{[\mathbf{p}]}\right\}\right|_{\mathcal{K}} \\
& +\left.\iint_{0}^{2 \pi} \sum_{s \geq 0}\left[\frac{\delta}{\delta \varphi^{j}(\theta, W)} \iint_{0}^{2 \pi} \epsilon^{s} A_{s}^{\nu \mu}\left(\boldsymbol{\varphi}\left(\theta^{\prime}, X\right), \epsilon \boldsymbol{\varphi}_{X}\left(\theta^{\prime}, X\right), \ldots\right) q_{\nu}(X) p_{\mu, s X}(X) \frac{\mathrm{d} \theta^{\prime}}{2 \pi} \mathrm{d} X\right]\right|_{\mathcal{K}} \\
& \quad \times g^{j}(\theta, W) \frac{\mathrm{d} \theta}{2 \pi} \mathrm{d} W+O\left(\mathbf{g}^{2}\right),
\end{aligned}
$$

where the values $\left.\left\{J_{[\mathbf{q}]}, J_{[\mathbf{p}]}\right\}\right|_{\mathcal{K}}$ as well as all the other values on $\mathcal{K}$ are calculated on the functions $\tilde{\varphi}^{i}(\theta, W) \in \mathcal{K}$ corresponding to the same values of the functionals $[\mathbf{U}(Z)]$ as the original functions $\varphi^{i}(\theta, W)$. We can see that the first term of the expansion of $\left\{J_{[\mathbf{q}]}, J_{[\mathbf{p}]}\right\}$ near $\mathcal{K}$ also has the order $O(\epsilon)$ as well as the values $\left\{J_{[\mathbf{q}]}, J_{[\mathbf{p}]}\right\}$ on $\mathcal{K}$ view the condition (2.35).

Considering relations (2.36), we can write a similar representation for the remaining terms of the expression (2.77).

We can see that the last term of the right-hand side of (2.77) has the order $O\left(\epsilon^{2}\right)$ in the whole space which gives the same order in $\epsilon$ for the terms of its expansion after the substitution of (2.78).

By Lemma 2.4, the second and the third terms of (2.77) are of the order $O\left(\epsilon^{2}\right)$ on the submanifold $\mathcal{K}$. However, this can not be said about the next terms of the expansion of these brackets in the values of $\mathbf{g}$. To find the values of these terms near $\mathcal{K}$ we write e.g.

$$
\begin{aligned}
& \iint_{0}^{2 \pi} B_{i[\mathbf{q}](1)}\left(\frac{S(Z)}{\epsilon}+\theta, Z\right) g^{i}(\theta, Z) \frac{\mathrm{d} \theta}{2 \pi} \mathrm{d} Z \\
& =\iint_{0}^{2 \pi} B_{i[\mathbf{q}](1)}\left(\frac{S(Z)}{\epsilon}+\theta, Z\right) \varphi^{i}(\theta, Z) \frac{\mathrm{d} \theta}{2 \pi} \mathrm{d} Z-\iint_{0}^{2 \pi} B_{i[\mathbf{q}](1)}(\theta, Z) \Phi^{i}(\theta, \mathbf{U}(Z)) \frac{\mathrm{d} \theta}{2 \pi} \mathrm{d} Z .
\end{aligned}
$$

After rather simple calculations it's not difficult to get then for the second term of (2.77) near $\mathcal{K}$

$$
\begin{aligned}
& \iint_{0}^{2 \pi}\left\{B_{i[\mathbf{q}](1)}\left(\frac{S(Z)}{\epsilon}+\theta, Z\right) g^{i}(\theta, Z), J_{[\mathbf{p}]}\right\} \frac{\mathrm{d} \theta}{2 \pi} \mathrm{d} Z \\
& =\left.\iint_{0}^{2 \pi}\left\{B_{i[\mathbf{q}](1)}\left(\frac{S(Z)}{\epsilon}+\theta, Z\right) g^{i}(\theta, Z), J_{[\mathbf{p}]}\right\}\right|_{\mathcal{K}} \frac{\mathrm{d} \theta}{2 \pi} \mathrm{d} Z \\
& +\int\left[\left.\int B_{i[\mathbf{q}](1)}\left(\frac{S(Z)}{\epsilon}+\theta, Z\right) \frac{\delta C_{(0)}^{i \mu}\left(\varphi(\theta, Z), \epsilon \varphi_{Z}(\theta, Z), \ldots\right)}{\delta \varphi^{j}\left(\theta^{\prime}, W\right)}\right|_{\mathcal{K}} p_{\mu}(Z) \frac{\mathrm{d} \theta}{2 \pi} \mathrm{d} Z+O(\epsilon)\right] \\
& \quad \times g^{j}\left(\theta^{\prime}, W\right) \frac{\mathrm{d} \theta^{\prime}}{2 \pi} \mathrm{d} W
\end{aligned}
$$




$$
\begin{aligned}
& +\int\left[\left.\int B_{i[\mathbf{q}](1) \theta}\left(\frac{S(Z)}{\epsilon}+\theta, Z\right) \frac{1}{2 \epsilon} \operatorname{sgn}(Z-W)\left\{k(W), J_{[\mathbf{p}]}\right\}\right|_{\mathcal{K}} \mathrm{d} W+O(\epsilon)\right] \\
& \times g^{i}(\theta, Z) \frac{\mathrm{d} \theta}{2 \pi} \mathrm{d} Z+O\left(\mathbf{g}^{2}\right) \\
& =\left.\iint_{0}^{2 \pi}\left\{B_{i[\mathbf{q}](1)}\left(\frac{S(Z)}{\epsilon}+\theta, Z\right) g^{i}(\theta, Z), J_{[\mathbf{p}]}\right\}\right|_{\mathcal{K}} \frac{\mathrm{d} \theta}{2 \pi} \mathrm{d} Z \\
& +\int\left[\left.\int B_{j[\mathbf{q}](1)}\left(\frac{S(Z)}{\epsilon}+\theta^{\prime}, Z\right) \frac{\delta S^{j \mu}\left(\boldsymbol{\varphi}\left(\theta^{\prime}, Z\right), \epsilon \boldsymbol{\varphi}_{Z}\left(\theta^{\prime}, Z\right), \ldots\right)}{\delta \varphi^{i}(\theta, W)}\right|_{\mathcal{K}} p_{\mu}(Z) \frac{\mathrm{d} \theta^{\prime}}{2 \pi} \mathrm{d} Z+O(\epsilon)\right] \\
& +\int g^{i}(\theta, W) \frac{\mathrm{d} \theta}{2 \pi} \mathrm{d} W \\
& \left.+\int B_{i[\mathbf{q}](1) \theta}\left(\frac{S(Z)}{\epsilon}+\theta, Z\right) \omega^{\mu}(Z) p_{\mu}(Z)+O(\epsilon)\right] g^{i}(\theta, Z) \frac{\mathrm{d} \theta}{2 \pi} \mathrm{d} Z+O\left(\mathbf{g}^{2}\right) .
\end{aligned}
$$

In general, considering the expansion of the brackets $(2.77)$ in the neighborhood of $\mathcal{K}$, we can write the relations

$$
\begin{aligned}
& \left\{\tilde{J}_{(1)[\mathbf{q}]}, \tilde{J}_{(1)[\mathbf{p}]}\right\}=\left.\left\{\tilde{J}_{(1)[\mathbf{q}]}, \tilde{J}_{(1)[\mathbf{p}]}\right\}\right|_{\mathcal{K}} \\
& \quad+\epsilon \iint_{0}^{2 \pi} \tilde{T}_{i[\mathbf{q}, \mathbf{p}]}\left(\frac{S(W)}{\epsilon}+\theta, W, \epsilon\right) g^{i}(\theta, W) \frac{\mathrm{d} \theta}{2 \pi} \mathrm{d} W+O\left(\mathbf{g}^{2}\right)
\end{aligned}
$$

with some regular at $\epsilon \rightarrow 0,2 \pi$-periodic in $\theta$, functions $\tilde{T}_{i[\mathbf{q}, \mathbf{p}]}(\theta, W, \epsilon)$.

Now for the bracket (2.27) we consider at the "points" of $\mathcal{K}$ the Jacobi identity of the form

$$
\left\{\left\{\tilde{J}_{(1)[\mathbf{q}]}, \tilde{J}_{(1)[\mathbf{p}]}\right\}, \tilde{J}_{(1)[\mathbf{r}]}\right\}+\text { c.p. } \equiv 0
$$

with some smooth compactly supported $\mathbf{q}(X), \mathbf{p}(X), \mathbf{r}(X)$.

We have on $\mathcal{K}$ the relations

$$
\left.\left\{g^{i}(\theta, W), \tilde{J}_{(1)[\mathbf{r}]}\right\}\right|_{\mathcal{K}}=O\left(\epsilon^{2}\right)
$$

for the bracket of the functionals $g^{i}(\theta, W)$ and $\tilde{J}_{(1)[\mathbf{r}]}$.

It's not difficult to get also

$$
\begin{aligned}
\left.\left\{U^{\gamma}(W), \tilde{J}_{(1)[\mathbf{r}]}\right\}\right|_{\mathcal{K}} & =\left.\left\{J^{\gamma}(W), \tilde{J}_{(1)[\mathbf{r}]}\right\}\right|_{\mathcal{K}}+O\left(\epsilon^{2}\right) \\
& =\left.\left\{J^{\gamma}(W), J_{[\mathbf{r}]}\right\}\right|_{\mathcal{K}}+O\left(\epsilon^{2}\right)=\epsilon\left\{U^{\gamma}(W), U_{[\mathbf{r}]}\right\}_{\mathrm{DN}}+O\left(\epsilon^{2}\right) .
\end{aligned}
$$

Now using relations (2.76) and expansion (2.79), we can see that the Jacobi identities (2.80) on $\mathcal{K}$ coincide in the leading order in $\epsilon\left(\epsilon^{2}\right)$ with the similar Jacobi identities for the DubrovinNovikov bracket

$$
\int \frac{\delta\left\{U_{[\mathbf{q}]}, U_{[\mathbf{p}]}\right\}_{\mathrm{DN}}}{\delta U^{\gamma}(W)}\left\{U^{\gamma}(W), U_{[\mathbf{r}]}\right\}_{\mathrm{DN}} \mathrm{d} W+\text { c.p. } \equiv 0
$$

on the space of fields $\mathbf{U}(X)$.

Let us formulate here also the Theorems justifying the invariance of the Dubrovin-Novikov procedure and the Hamiltonian properties of the Whitham system (2.10) with respect to the averaged bracket in the single-phase case.

Theorem 2.2. Let $\Lambda$ be a regular Hamiltonian family of single-phase solutions of (2.3). Let $\left(I^{1}, \ldots, I^{N}\right)$ and $\left(I^{\prime 1}, \ldots, I^{\prime N}\right)$ be two different complete Hamiltonian sets of commuting first integrals of (2.3) having the form (2.18). Then the Dubrovin-Novikov brackets obtained using the sets $\left(I^{1}, \ldots, I^{N}\right)$ and $\left(I^{\prime 1}, \ldots, I^{N}\right)$ coincide with each other. 
Theorem 2.3. Let $\Lambda$ be a regular Hamiltonian family of single-phase solutions of (2.3). Let $\left(I^{1}, \ldots, I^{N}\right)$ be a complete Hamiltonian set of commuting first integrals of (2.3) having the form (2.18) and $H$ be the Hamiltonian function for the system (2.3) having the form (2.17). Then the Whitham system (2.10) is Hamiltonian with respect to the corresponding DubrovinNovikov bracket (2.21) with the Hamiltonian function (2.22)

$$
H^{a v}=\int_{-\infty}^{+\infty}\left\langle P_{H}\right\rangle(\mathbf{U}(X)) \mathrm{d} X
$$

Proofs of Theorems 2.2 and 2.3 are identical for the single-phase and the multiphase cases, so we prove them in general $m$-phase case in the next chapter.

\section{Dubrovin-Novikov bracket and the multiphase case}

We turn now to the general multiphase situation. We note that the difference with the singlephase case occur only in Theorem 2.1, since finding the functions $B_{j[\mathbf{q}](1)}(\boldsymbol{\theta}, X)$ here is a more complicated problem. Let us return to the system (2.62), i.e.

$$
\hat{B}_{[0]}^{i j}(X) B_{j[\mathbf{q}](1)}(\boldsymbol{\theta}, X)+A_{[1][\mathbf{q}]}^{i}(\boldsymbol{\theta}, X)=0,
$$

where the operator $\hat{B}_{[0]}^{i j}(X)$ and the functions $A_{[1][\mathbf{q}]}^{i}(\boldsymbol{\theta}, X)$ are given by (2.63) and (2.64) respectively. It is easy to see that the operator $\hat{B}_{[0]}^{i j}(X)$ is a differential operator on the torus, which acts along a constant direction $k^{\alpha} \partial / \partial \theta^{\alpha}$. The corresponding vector field generates one-dimensional trajectories which are everywhere dense in the torus $\mathbb{T}^{m}$ for generic vectors $\mathbf{k}(X)$. For special values of $\mathbf{k}(X)$ the closures of the trajectories may be lower-dimensional tori, in particular, the closed one-dimensional trajectories in $\mathbb{T}^{m}$. Note that the operator $\hat{B}_{[0]}^{i j}(X) \equiv \hat{B}_{[0]}^{i j}(\mathbf{U}(X))$ is exactly the Hamiltonian operator for the bracket (2.16) on the manifold of $m$-phase solutions $\Lambda$.

The operator $\hat{B}_{[0]}^{i j}(\mathbf{U})$ has in general finite number of "regular" eigenvectors with zero eigenvalues defined for all values of the parameters $\mathbf{U}$ and smoothly depending on the parameters. However, for special values of $\mathbf{U}$ the set of eigenvectors with zero eigenvalues is infinite and determined by the dimension of closures of the straight-line trajectories in $\mathbb{T}^{m}$, defined by the vector $\mathbf{k}(\mathbf{U}) .^{3}$

Let us define in the space of the parameters $\mathbf{U}$ the set $\mathcal{M}$, such that for all $\mathbf{U} \in \mathcal{M}$ the trajectories of the vector field $\left(k^{1}(\mathbf{U}), \ldots, k^{m}(\mathbf{U})\right)$ are everywhere dense in $\mathbb{T}^{m}$. From the condition

$$
\operatorname{rank}\left\|\partial k^{\alpha} / \partial U^{\nu}\right\|=m
$$

it follows that the set $\mathcal{M}$ is everywhere dense in the parameter space $\mathbf{U}$ and, moreover, has the full measure.

\footnotetext{
${ }^{3}$ For some special brackets $(2.16)$ the differential part can be absent in the operator $\hat{B}_{[0]}^{i j}(\mathbf{U})$. The operator $\hat{B}_{[0]}^{i j}(\mathbf{U})$ reduces then to an ultralocal operator acting independently at every point of $\mathbb{T}^{m}$. As a rule, the matrix $B_{[0]}^{i j}(\mathbf{U})$ is non-degenerate in this case. For example, for the ultralocal Poisson bracket

$$
\{\psi(x), \bar{\psi}(y)\}=i \delta(x-y)
$$

for the NLS equation

$$
i \psi_{t}=\psi_{x x}+\nu|\psi|^{2} \psi
$$

we have exactly this situation. Easy to see that system (3.1) represents a simple algebraic system in this case and is trivially solvable. The multiphase situation is not different here from the single-phase one, so all the arguments of the previous chapter can be used also for the multiphase case. However, for arbitrary brackets (2.16) the operators $\hat{B}_{[0]}^{i j}(\mathbf{U})$ have more general form described above.
} 
In the study of the solubility of (3.1) we must first require the orthogonality of the functions $A_{[1][\mathbf{q}]}^{i}(\boldsymbol{\theta}, X)$ to the "regular" eigenvectors of $\hat{B}_{[0]}^{i j}(X)$ with zero eigenvalues. Let us prove here the following lemma.

Lemma 3.1. Let $\Lambda$ be a regular Hamiltonian family of $m$-phase solutions of $(2.3)$ and $\left(I^{1}, \ldots\right.$, $\left.I^{N}\right)$ be a complete Hamiltonian set of commuting first integrals of (2.3) having the form (2.18). Let for $\mathbf{U} \in \mathcal{M}$

$$
\mathbf{v}_{[\mathbf{U}]}^{(l)}(\boldsymbol{\theta})=\left(v_{1[\mathbf{U}]}^{(l)}(\boldsymbol{\theta}), \ldots, v_{n[\mathbf{U}]}^{(l)}(\boldsymbol{\theta})\right), \quad l=1, \ldots, s
$$

be the complete set of linearly independent eigenvectors of the operator $\hat{B}_{[0]}^{i j}(\mathbf{U})$ on the torus with zero eigenvalues, smoothly depending on $\boldsymbol{\theta}$. Then

1) the number of the vectors $\mathbf{v}_{[\mathbf{U}]}^{(l)}(\boldsymbol{\theta})$ is equal to the number of annihilators of the bracket (2.16) on the submanifold of $m$-phase solutions of (2.3);

2) the functions $A_{[1][\mathbf{q}]}^{i}(\boldsymbol{\theta}, X)$ are orthogonal to all the vectors $\mathbf{v}_{[\mathbf{U}(X)]}^{(l)}(\boldsymbol{\theta})$, i.e.

$$
\int_{0}^{2 \pi} \cdots \int_{0}^{2 \pi} v_{i[\mathbf{U}(X)]}^{(l)}(\boldsymbol{\theta}) A_{[1][\mathbf{q}]}^{i}(\boldsymbol{\theta}, X) \frac{\mathrm{d}^{m} \theta}{(2 \pi)^{m}} \equiv 0 .
$$

Proof. Consider the values of $\mathbf{v}_{[\mathbf{U}]}^{(l)}(\boldsymbol{\theta})$ on any of the trajectories of the vector field $k^{\alpha} \partial / \partial \theta^{\alpha}$ on the torus $\mathbb{T}^{m}$. According to the definition of regular Hamiltonian family of $m$-phase solutions of (2.3) the corresponding functions $v_{i[\mathbf{U}]}^{(l)}\left(\mathbf{k} x+\boldsymbol{\theta}_{0}\right)$ should be the variational derivatives of some linear combination of annihilators of the bracket (2.16). We have then for a fixed value of $\boldsymbol{\theta}_{0}$

$$
v_{i[\mathbf{U}]}^{(l)}\left(\mathbf{k} x+\boldsymbol{\theta}_{0}\right)=\left.\sum_{p} \alpha_{p}^{l}\left(\mathbf{U}, \boldsymbol{\theta}_{0}\right) \frac{\delta N^{p}}{\delta \varphi^{i}(x)}\right|_{\boldsymbol{\varphi}=\mathbf{\Phi}\left(\mathbf{k} x+\boldsymbol{\theta}_{0}, \mathbf{U}\right)} .
$$

From relation (2.26) we have then

$$
v_{i[\mathbf{U}]}^{(l)}\left(\mathbf{k}(\mathbf{U}) x+\boldsymbol{\theta}_{0}\right)=\sum_{p, q} \alpha_{p}^{l}\left(\mathbf{U}, \boldsymbol{\theta}_{0}\right) n_{q}^{p}(\mathbf{U}) \kappa_{i[\mathbf{U}]}^{(q)}\left(\mathbf{k}(\mathbf{U}) x+\boldsymbol{\theta}_{0}\right) .
$$

By definition, for $\mathbf{U} \in \mathcal{M}$ the trajectories of the field $k^{\alpha} \partial / \partial \theta^{\alpha}$ are everywhere dense in $\mathbb{T}^{m}$. Since both the left- and the right-hand parts of (3.2) are smooth functions on $\mathbb{T}^{m}$, we get then that they coincide on $\mathbb{T}^{m}$. We can put then $\alpha_{p}^{l}\left(\mathbf{U}, \boldsymbol{\theta}_{0}\right)=\alpha_{p}^{l}(\mathbf{U})$ and write

$$
v_{i[\mathbf{U}]}^{(l)}(\boldsymbol{\theta}) \equiv \sum_{p, q} \alpha_{p}^{l}(\mathbf{U}) n_{q}^{p}(\mathbf{U}) \kappa_{i[\mathbf{U}]}^{(q)}(\boldsymbol{\theta}) .
$$

It is easy to see also that any linear combination of the form (3.3) gives a regular eigenvector of the operator $\hat{B}_{[0]}^{i j}(\mathbf{U}(X))$ with zero eigenvalue.

Statement (2) follows then from relation (2.71) in view of representation (3.3).

However, despite the presence of Lemma 3.1, study of system (3.1) is much more complicated in the multiphase case if compared with the single-phase case. Thus, the presence of "resonances" for some values of the parameters $\mathbf{U}(X)$ may lead to insolubility of (3.1) in the space of periodic (in all $\theta^{\alpha}$ ) functions. The set of the "resonance" values of $\mathbf{U}$, as a rule, has measure zero. Nevertheless, the possibility of resonant parameters do not allow to transfer directly the methods of the previous chapter to the multiphase case. The following theorem shows, however, that the procedure of the bracket averaging is in fact insensitive to the appearance of the resonant values of $\mathbf{U}$ and can be used in most multiphase cases, as well as in the single-phase case. 
Theorem 3.1. Let the system (2.3) be a local Hamiltonian system generated by the functional (2.17) in the local field-theoretic Hamiltonian structure (2.16). Let $\Lambda$ be a regular Hamiltonian family of $m$-phase solutions of $(2.3)$ and $\left(I^{1}, \ldots, I^{N}\right)$ be a complete Hamiltonian set of commuting integrals (2.18) for this family.

Let the parameter space $\mathbf{U}$ of the family $\Lambda$ have a dense set $\mathcal{S} \subset \mathcal{M}$ on which the system (3.1), or, equivalently, the multiphase system (2.72), (2.73) is solvable in the space of smooth $2 \pi-$ periodic in each $\theta^{\alpha}$ functions. Then the Dubrovin-Novikov bracket, obtained with the aid of the functionals $\left(I^{1}, \ldots, I^{N}\right)$, satisfies the Jacobi identity.

Proof. As before, for a smooth compactly supported vector-valued function $\mathbf{q}(X)=\left(q_{1}(X)\right.$, $\left.\ldots, q_{N}(X)\right)$ we define the functional

$$
J_{[\mathbf{q}]}=\int_{-\infty}^{+\infty} J^{\nu}(X) q_{\nu}(X) \mathrm{d} X .
$$

Then, for arbitrary smooth, compactly supported in $X$ and $2 \pi$-periodic in each $\theta^{\alpha}$ functions $\tilde{\mathbf{Q}}(\boldsymbol{\theta}, X)=\left(\tilde{Q}_{1}(\boldsymbol{\theta}, X), \ldots, \tilde{Q}_{n}(\boldsymbol{\theta}, X)\right)$ we define the functionals

$$
\begin{aligned}
Q_{i}(\boldsymbol{\theta}, X)= & \tilde{Q}_{i}(\boldsymbol{\theta}, X) \\
& -\Phi_{\theta^{\beta}}^{i}(\boldsymbol{\theta}, \mathbf{U}(X)) M^{\beta \gamma}(\mathbf{U}(X)) \int_{0}^{2 \pi} \cdots \int_{0}^{2 \pi} \tilde{Q}_{j}\left(\boldsymbol{\theta}^{\prime}, X\right) \Phi_{\theta^{\prime} \gamma}^{j}\left(\boldsymbol{\theta}^{\prime}, \mathbf{U}(X)\right) \frac{\mathrm{d}^{m} \theta^{\prime}}{(2 \pi)^{m}},
\end{aligned}
$$

where the matrix $M^{\beta \gamma}(\mathbf{U})$ is the inverse of the matrix

$$
\int_{0}^{2 \pi} \cdots \int_{0}^{2 \pi} \sum_{i=1}^{n} \Phi_{\theta^{\beta}}^{i}(\boldsymbol{\theta}, \mathbf{U}) \Phi_{\theta^{\gamma}}^{i}(\boldsymbol{\theta}, \mathbf{U}) \frac{\mathrm{d}^{m} \theta}{(2 \pi)^{m}},
$$

which is always defined according to the definition of a complete regular family of $m$-phase solutions of system (2.3).

By definition, the functions $Q_{i}(\boldsymbol{\theta}, X)$ are local functionals of $\mathbf{U}(X)$

$$
Q_{i}(\boldsymbol{\theta}, X) \equiv Q_{i}(\boldsymbol{\theta}, X, \mathbf{U}(X))
$$

depending also on the arbitrary fixed functions $\tilde{\mathbf{Q}}(\boldsymbol{\theta}, X)$. Everywhere below we will assume that $\mathbf{Q}(\boldsymbol{\theta}, X)$ is a functional of this type defined with some function $\tilde{\mathbf{Q}}(\boldsymbol{\theta}, X)$.

Easy to see that the values of $Q_{i}(\boldsymbol{\theta}, X)$ with arbitrary $\tilde{\mathbf{Q}}(\boldsymbol{\theta}, X)$ represent for fixed values of the functionals $\mathbf{U}(Z)$ all possible smooth, compactly supported in $X$ and $2 \pi$-periodic in each $\theta^{\alpha}$ functions with the only restriction

$$
\int_{0}^{2 \pi} \cdots \int_{0}^{2 \pi} Q_{i}(\boldsymbol{\theta}, X) \Phi_{\theta^{\alpha}}^{i}(\boldsymbol{\theta}, \mathbf{U}(X)) \frac{\mathrm{d}^{m} \theta}{(2 \pi)^{m}}=0, \quad \forall X, \alpha=1, \ldots, m .
$$

For the functionals $Q_{i}(\boldsymbol{\theta}, X)$ we define the functionals

$$
g_{[\mathbf{Q}]}=\int_{-\infty}^{+\infty} \int_{0}^{2 \pi} \cdots \int_{0}^{2 \pi} g^{i}(\boldsymbol{\theta}, X) Q_{i}\left(\frac{\mathbf{S}(X)}{\epsilon}+\boldsymbol{\theta}, X\right) \frac{\mathrm{d}^{m} \theta}{(2 \pi)^{m}} \mathrm{~d} X .
$$

Now, for fixed functions $\mathbf{q}(X), \mathbf{p}(X)$, and functional $\mathbf{Q}(\boldsymbol{\theta}, X)$ consider the Jacobi identity of the form

$$
\left\{g_{[\mathbf{Q}]},\left\{J_{[\mathbf{q}]}, J_{[\mathbf{p}]}\right\}\right\}+\left\{J_{[\mathbf{p}]},\left\{g_{[\mathbf{Q}]}, J_{[\mathbf{q}]}\right\}\right\}+\left\{J_{[\mathbf{q}]},\left\{J_{[\mathbf{p}]}, g_{[\mathbf{Q}]}\right\}\right\} \equiv 0
$$


Expanding the values of the brackets $\left\{J^{\nu}(X), J^{\mu}(Y)\right\}$ in the neighborhood of the submanifold $\mathcal{K}$, as in the single-phase case, we can write

$$
\begin{aligned}
\left\{J^{\nu}(X), J^{\mu}(Y)\right\}=\left.\left\{J^{\nu}(X), J^{\mu}(Y)\right\}\right|_{\mathcal{K}} \\
+\left.\sum_{s \geq 0}\left[\frac{\delta}{\delta \varphi^{k}(\boldsymbol{\theta}, W)} \int_{0}^{2 \pi} \cdots \int_{0}^{2 \pi} A_{s}^{\nu \mu}\left(\boldsymbol{\varphi}\left(\boldsymbol{\theta}^{\prime}, X\right), \epsilon \boldsymbol{\varphi}_{X}\left(\boldsymbol{\theta}^{\prime}, X\right), \ldots\right) \frac{\mathrm{d}^{m} \theta^{\prime}}{(2 \pi)^{m}}\right]\right|_{\mathcal{K}} \\
\times g^{k}(\boldsymbol{\theta}, W) \frac{\mathrm{d}^{m} \theta}{(2 \pi)^{m}} \mathrm{~d} W \epsilon^{s} \delta^{(s)}(X-Y)+O\left(\mathbf{g}^{2}\right),
\end{aligned}
$$

where all the values on the submanifold $\mathcal{K}$ are calculated at the same values of the functionals $[\mathbf{U}(Z)]$ as for the original function $\boldsymbol{\varphi}(\boldsymbol{\theta}, X)$.

Let us introduce by definition

$$
\begin{aligned}
& \left.\frac{\delta\left\{J_{[\mathbf{q}]}, J_{[\mathbf{p}]}\right\}}{\delta g^{k}(\boldsymbol{\theta}, W)}\right|_{\mathcal{K}} \\
& \left.\equiv \sum_{s \geq 0} \int\left[\frac{\delta}{\delta \varphi^{k}(\boldsymbol{\theta}, W)} \int_{0}^{2 \pi} \cdots \int_{0}^{2 \pi} \epsilon^{s} A_{s}^{\nu \mu}\left(\boldsymbol{\varphi}\left(\boldsymbol{\theta}^{\prime}, X\right), \epsilon \boldsymbol{\varphi}_{X}\left(\boldsymbol{\theta}^{\prime}, X\right), \ldots\right) \frac{\mathrm{d}^{m} \theta^{\prime}}{(2 \pi)^{m}}\right]\right|_{\mathcal{K}} q_{\nu}(X) p_{\mu, s X} \mathrm{~d} X
\end{aligned}
$$

Note that the notations $\delta / \delta g^{k}(\boldsymbol{\theta}, W)$, generally speaking, are not natural in our situation because of the dependence of the chosen system of constraints. Nevertheless, the preservation of these notations can better clarify the algebraic structure of the further calculations.

The values defined by (3.6) can be represented in the form of the graded decompositions at $\epsilon \rightarrow 0$ on the submanifold $\mathcal{K}$. By virtue of (2.35) it is easy to conclude that the expansion in $\epsilon$ of the quantities (3.6) begins with the first degree in $\epsilon$

$$
\left.\frac{\delta\left\{J_{[\mathbf{q}]}, J_{[\mathbf{p}]}\right\}}{\delta g^{k}(\boldsymbol{\theta}, W)}\right|_{\mathcal{K}}=\left.\epsilon \frac{\delta\left\{J_{[\mathbf{q}]}, J_{[\mathbf{p}]}\right\}}{\delta g^{k}(\boldsymbol{\theta}, W)}\right|_{\mathcal{K}[1]}+\left.\epsilon^{2} \frac{\delta\left\{J_{[\mathbf{q}]}, J_{[\mathbf{p}]}\right\}}{\delta g^{k}(\boldsymbol{\theta}, W)}\right|_{\mathcal{K}[2]}+\cdots
$$

The leading term of (3.7) can be divided into two parts, corresponding to the functions $\epsilon Q_{X}^{\nu \mu}\left(\boldsymbol{\varphi}, \epsilon \boldsymbol{\varphi}_{X}, \ldots\right)$ and $A_{1}^{\nu \mu}\left(\boldsymbol{\varphi}, \epsilon \boldsymbol{\varphi}_{X}, \ldots\right)$ and containing the quantities $q_{\nu}(W) p_{\mu}(W)$ and $q_{\nu}(W) p_{\mu, W}(W)$ as local factors, respectively.

The values of $\left\{J_{[\mathbf{q}]}, J_{[\mathbf{p}]}\right\}$, are obviously invariant under transformations of the form

$$
\varphi(\theta, X) \rightarrow \varphi(\theta+\Delta \theta, X) .
$$

From the invariance of the functionals $\mathbf{U}(X)$ in such transformations, we can write for the corresponding increments of the constraints $(2.32)$

$$
\delta g^{k}(\boldsymbol{\theta}, X)=\varphi^{k}(\boldsymbol{\theta}+\delta \boldsymbol{\theta}, X)-\varphi^{k}(\boldsymbol{\theta}, X) .
$$

As a consequence, we can write on the submanifold $\mathcal{K}$

$$
\begin{aligned}
& \left.\iint_{0}^{2 \pi} \cdots \int_{0}^{2 \pi} \frac{\delta\left\{J_{[\mathbf{q}]}, J_{[\mathbf{p}]}\right\}}{\delta g^{k}(\boldsymbol{\theta}, W)}\right|_{\mathcal{K}} \Phi_{\theta^{\alpha}}^{k}\left(\frac{\mathbf{S}(W)}{\epsilon}+\boldsymbol{\theta}, \mathbf{U}(W)\right) \frac{\mathrm{d}^{m} \theta}{(2 \pi)^{m}} \mathrm{~d} W \equiv 0, \\
& \alpha=1, \ldots, m .
\end{aligned}
$$

The above relation is satisfied to all orders in $\epsilon$. By the arbitrariness of the functions $q_{\nu}(X)$, the relation (3.9) in the leading order can be strengthened. Namely, according to the remark about the form of the leading term of (3.7) we can write for any $W$

$$
\left.\int_{0}^{2 \pi} \cdots \int_{0}^{2 \pi} \frac{\delta\left\{J_{[\mathbf{q}]}, J_{[\mathbf{p}]}\right\}}{\delta g^{k}(\boldsymbol{\theta}, W)}\right|_{\mathcal{K}[1]} \Phi_{\theta^{\alpha}}^{k}\left(\frac{\mathbf{S}(W)}{\epsilon}+\boldsymbol{\theta}, \mathbf{U}(W)\right) \frac{\mathrm{d}^{m} \theta}{(2 \pi)^{m}} \equiv 0, \quad \alpha=1, \ldots, m
$$


Quite similarly, we have the relation

$$
\begin{aligned}
\left\{g_{[\mathbf{Q}]},\right. & \left.J_{[\mathbf{q}]}\right\}=\int Q_{i}\left(\frac{\mathbf{S}(X)}{\epsilon}+\boldsymbol{\theta}, X\right)\left\{g^{i}(\boldsymbol{\theta}, X), J_{[\mathbf{q}]}\right\} \frac{\mathrm{d}^{m} \theta}{(2 \pi)^{m}} \mathrm{~d} X \\
+ & \int g^{i}(\boldsymbol{\theta}, X) Q_{i, \theta^{\alpha}}\left(\frac{\mathbf{S}(X)}{\epsilon}+\boldsymbol{\theta}, X\right) \frac{1}{2 \epsilon} \operatorname{sgn}(X-Y)\left\{k^{\alpha}(Y), J_{[\mathbf{q}]}\right\} \frac{\mathrm{d}^{m} \theta}{(2 \pi)^{m}} \mathrm{~d} Y \mathrm{~d} X \\
& +\int g^{i}(\boldsymbol{\theta}, X) Q_{i, U^{\nu}}\left(\frac{\mathbf{S}(X)}{\epsilon}+\boldsymbol{\theta}, X\right)\left\{U^{\nu}(X), J_{[\mathbf{q}]}\right\} \frac{\mathrm{d}^{m} \theta}{(2 \pi)^{m}} \mathrm{~d} X \\
= & \int Q_{i}\left(\frac{\mathbf{S}(X)}{\epsilon}+\boldsymbol{\theta}, X\right) \sum_{l \geq 0} \epsilon^{l} C_{(l)}^{i \mu}\left(\boldsymbol{\varphi}(\boldsymbol{\theta}, X), \epsilon \boldsymbol{\varphi}_{X}(\boldsymbol{\theta}, X), \ldots\right) \frac{\mathrm{d}^{m} \theta}{(2 \pi)^{m}} q_{\mu, l X}(X) \mathrm{d} X \\
& -\int Q_{i}\left(\frac{\mathbf{S}(X)}{\epsilon}+\boldsymbol{\theta}, X\right) \Phi_{U^{\nu}}^{i}\left(\frac{\mathbf{S}(X)}{\epsilon}+\boldsymbol{\theta}, \mathbf{U}(X)\right)\left\{U^{\nu}(X), J_{[\mathbf{q}]}\right\} \frac{\mathrm{d}^{m} \theta}{(2 \pi)^{m}} \mathrm{~d} X \\
& -\int Q_{i}\left(\frac{\mathbf{S}(X)}{\epsilon}+\boldsymbol{\theta}, X\right) \Phi_{\theta^{\alpha}}^{i}\left(\frac{\mathbf{S}(X)}{\epsilon}+\boldsymbol{\theta}, \mathbf{U}(X)\right) \\
& \times \frac{1}{2 \epsilon} \operatorname{sgn}(X-Y)\left\{k^{\alpha}(Y), J_{[\mathbf{q}]}\right\} \frac{\mathrm{d}^{m} \theta}{(2 \pi)^{m}} \mathrm{~d} Y \mathrm{~d} X \\
+ & \int g^{i}(\boldsymbol{\theta}, X) Q_{i, \theta^{\alpha}}\left(\frac{\mathbf{S}(X)}{\epsilon}+\boldsymbol{\theta}, X\right) \frac{1}{2 \epsilon} \operatorname{sgn}(X-Y)\left\{k^{\alpha}(Y), J_{[\mathbf{q}]}\right\} \frac{\mathrm{d}^{m} \theta}{(2 \pi)^{m}} \mathrm{~d} Y \mathrm{~d} X \\
+ & \int g^{i}(\boldsymbol{\theta}, X) Q_{i, U^{\nu}}\left(\frac{\mathbf{S}(X)}{\epsilon}+\boldsymbol{\theta}, X\right)\left\{U^{\nu}(X), J_{[\mathbf{q}]}\right\} \frac{\mathrm{d}^{m} \theta}{(2 \pi)^{m}} \mathrm{~d} X .
\end{aligned}
$$

According to relations (3.4), we can actually see here that the third term in the above expression is identically equal to zero.

According to the form of the constraints, we have again in the neighborhood of $\mathcal{K}$

$$
\begin{aligned}
& \left\{g_{[\mathbf{Q}]}, J_{[\mathbf{q}]}\right\}=\left.\left\{g_{[\mathbf{Q}]}, J_{[\mathbf{q}]}\right\}\right|_{\mathcal{K}} \\
& +\left.\left[\int Q_{i}\left(\frac{\mathbf{S}(X)}{\epsilon}+\boldsymbol{\theta}^{\prime}, X\right) \sum_{l \geq 0} \epsilon^{l} \frac{\delta C_{(l)}^{i \mu}\left(\boldsymbol{\varphi}\left(\boldsymbol{\theta}^{\prime}, X\right), \epsilon \boldsymbol{\varphi}_{X}\left(\boldsymbol{\theta}^{\prime}, X\right), \ldots\right)}{\delta \varphi^{k}(\boldsymbol{\theta}, W)} \frac{\mathrm{d}^{m} \theta^{\prime}}{(2 \pi)^{m}} q_{\mu, l X}(X) \mathrm{d} X\right]\right|_{\mathcal{K}} \\
& \quad \times g^{k}(\boldsymbol{\theta}, W) \frac{\mathrm{d}^{m} \theta}{(2 \pi)^{m}} \mathrm{~d} W \\
& -\left.\int Q_{i}\left(\boldsymbol{\theta}^{\prime}, X\right) \Phi_{U^{\nu}}^{i}\left(\boldsymbol{\theta}^{\prime}, \mathbf{U}(X)\right) \frac{\mathrm{d}^{m} \theta^{\prime}}{(2 \pi)^{m}} \frac{\delta\left\{U^{\nu}(X), J_{[\mathbf{q}]}\right\}}{\delta \varphi^{k}(\boldsymbol{\theta}, W)}\right|_{\mathcal{K}} \mathrm{d} X \times g^{k}(\boldsymbol{\theta}, W) \frac{\mathrm{d}^{m} \theta}{(2 \pi)^{m}} \mathrm{~d} W \\
& +\left.\int Q_{k, \theta^{\alpha}}\left(\frac{\mathbf{S}(W)}{\epsilon}+\boldsymbol{\theta}, W\right) \frac{1}{2 \epsilon} \operatorname{sgn}(W-Y)\left\{k^{\alpha}(Y), J_{[\mathbf{q}]}\right\}\right|_{\mathcal{K}} \mathrm{d} Y \times g^{k}(\boldsymbol{\theta}, W) \frac{\mathrm{d}^{m} \theta}{(2 \pi)^{m}} \mathrm{~d} W \\
& +\left.\int Q_{i, U^{\nu}}\left(\frac{\mathbf{S}(W)}{\epsilon}+\boldsymbol{\theta}, W\right)\left\{U^{\nu}(W), J_{[\mathbf{q}]}\right\}\right|_{\mathcal{K}} \times g^{k}(\boldsymbol{\theta}, W) \frac{\mathrm{d}^{m} \theta}{(2 \pi)^{m}} \mathrm{~d} W+O\left(\mathbf{g}^{2}\right)
\end{aligned}
$$

provided that all the values on the submanifold $\mathcal{K}$ are calculated at the same values of the functionals $[\mathbf{U}(Z)]$.

For the quantities $\left\{g_{[\mathbf{Q}]}, J_{[\mathbf{q}]}\right\}$ we can introduce by definition

$$
\begin{aligned}
& \left.\frac{\delta\left\{g_{[\mathbf{Q}]}, J_{[\mathbf{q}]}\right\}}{\delta g^{k}(\boldsymbol{\theta}, W)}\right|_{\mathcal{K}} \\
& \left.\equiv \int Q_{i}\left(\frac{\mathbf{S}(X)}{\epsilon}+\boldsymbol{\theta}^{\prime}, X\right)\left[\sum_{l \geq 0} \epsilon^{l} \frac{\delta C_{(l)}^{i \mu}\left(\boldsymbol{\varphi}\left(\boldsymbol{\theta}^{\prime}, X\right), \epsilon \boldsymbol{\varphi}_{X}\left(\boldsymbol{\theta}^{\prime}, X\right), \ldots\right)}{\delta \varphi^{k}(\boldsymbol{\theta}, W)} q_{\mu, l X}(X)\right]\right|_{\mathcal{K}} \frac{\mathrm{d}^{m} \theta^{\prime}}{(2 \pi)^{m}} \mathrm{~d} X \\
& \quad-\left.\int Q_{i}\left(\boldsymbol{\theta}^{\prime}, X\right) \Phi_{U^{\nu}}^{i}\left(\boldsymbol{\theta}^{\prime}, \mathbf{U}(X)\right) \frac{\mathrm{d}^{m} \theta^{\prime}}{(2 \pi)^{m}} \frac{\delta\left\{U^{\nu}(X), J_{[\mathbf{q}]}\right\}}{\delta \varphi^{k}(\boldsymbol{\theta}, W)}\right|_{\mathcal{K}} \mathrm{d} X
\end{aligned}
$$




$$
\begin{aligned}
& +\left.Q_{k, \theta^{\alpha}}\left(\frac{\mathbf{S}(W)}{\epsilon}+\boldsymbol{\theta}, W\right) \frac{1}{2 \epsilon} \int \operatorname{sgn}(W-Y)\left\{k^{\alpha}(Y), J_{[\mathbf{q}]}\right\}\right|_{\mathcal{K}} \mathrm{d} Y \\
& +\left.Q_{i, U^{\nu}}\left(\frac{\mathbf{S}(W)}{\epsilon}+\boldsymbol{\theta}, W\right)\left\{U^{\nu}(W), J_{[\mathbf{q}]}\right\}\right|_{\mathcal{K}} .
\end{aligned}
$$

The quantities $\delta\left\{g_{[\mathbf{Q}]}, J_{[\mathbf{q}]}\right\} /\left.\delta g^{k}(\boldsymbol{\theta}, W)\right|_{\mathcal{K}}$ have the order $O(1)$ at $\epsilon \rightarrow 0$. We have also

$$
C_{(0)}^{i \mu}\left(\boldsymbol{\varphi}\left(\boldsymbol{\theta}^{\prime}, X\right), \epsilon \boldsymbol{\varphi}_{X}\left(\boldsymbol{\theta}^{\prime}, X\right), \ldots\right) \equiv S^{i \mu}\left(\boldsymbol{\varphi}\left(\boldsymbol{\theta}^{\prime}, X\right), \epsilon \boldsymbol{\varphi}_{X}\left(\boldsymbol{\theta}^{\prime}, X\right), \ldots\right)
$$

according to relation (2.37).

Let us introduce the functions

$$
S_{k(l)}^{i \mu}\left(\boldsymbol{\varphi}, \boldsymbol{\varphi}_{x}, \ldots\right) \equiv \frac{\partial S^{i \mu}\left(\boldsymbol{\varphi}, \boldsymbol{\varphi}_{x}, \ldots\right)}{\partial \varphi_{l x}^{k}} .
$$

Using now Lemma 2.3 and relations (3.7) we can write for the leading term of (3.11)

$$
\begin{aligned}
\left.\frac{\delta\left\{g_{[\mathbf{Q}]}, J_{[\mathbf{q}]}\right\}}{\delta g^{k}(\boldsymbol{\theta}, W)}\right|_{\mathcal{K}[0]}= & \sum_{l \geq 0}(-1)^{l} k^{\alpha_{1}}(W) \cdots k^{\alpha_{l}}(W) \frac{\partial^{l}}{\partial \theta^{\alpha_{1}} \cdots \partial \theta^{\alpha_{l}}} \\
& \times\left[\left.Q_{i}\left(\frac{\mathbf{S}(W)}{\epsilon}+\boldsymbol{\theta}, W\right) S_{k(l)}^{i \mu}\left(\boldsymbol{\varphi}(\boldsymbol{\theta}, W), \epsilon \boldsymbol{\varphi}_{W}(\boldsymbol{\theta}, W), \ldots\right)\right|_{\mathcal{K}[0]}\right] q_{\mu}(W) \\
& +\omega^{\alpha \mu}(W) q_{\mu}(W) Q_{k, \theta^{\alpha}}\left(\frac{\mathbf{S}(W)}{\epsilon}+\boldsymbol{\theta}, W\right)
\end{aligned}
$$

where

$$
\begin{aligned}
S_{k(l)}^{i \mu} & \left.\left(\boldsymbol{\varphi}(\boldsymbol{\theta}, W), \epsilon \boldsymbol{\varphi}_{W}(\boldsymbol{\theta}, W), \ldots\right)\right|_{\mathcal{K}[0]} \\
& \equiv S_{k(l)}^{i \mu}\left(\boldsymbol{\Phi}\left(\frac{\mathbf{S}(W)}{\epsilon}+\boldsymbol{\theta}, \mathbf{U}(W)\right), k^{\gamma} \boldsymbol{\Phi}_{\theta^{\gamma}}\left(\frac{\mathbf{S}(W)}{\epsilon}+\boldsymbol{\theta}, \mathbf{U}(W)\right), \ldots\right) .
\end{aligned}
$$

Note one more property of the values $\delta\left\{g_{[\mathbf{Q}]}, J_{[\mathbf{q}]}\right\} /\left.\delta g^{k}(\boldsymbol{\theta}, W)\right|_{\mathcal{K}[0]}$. As we saw earlier, the values $\left\{g_{[\mathbf{Q}]}, J_{[\mathbf{q}]}\right\}$ are of the order $O(\epsilon)$ at $\epsilon \rightarrow 0$ on the submanifold $\mathcal{K}$. This property is preserved also under the overall shift of the initial phase (3.8).

Indeed, for

$$
\varphi^{i}(\boldsymbol{\theta}, X)=\Phi^{i}\left(\frac{\mathbf{S}(X)}{\epsilon}+\boldsymbol{\theta}+\Delta \boldsymbol{\theta}, \mathbf{U}(X)\right)
$$

we can write

$$
\begin{aligned}
\left\{g_{[\mathbf{Q}]}, J_{[\mathbf{q}]}\right\}= & \int Q_{i, \theta^{\alpha}}\left(\frac{\mathbf{S}(Z)}{\epsilon}+\boldsymbol{\theta}, Z\right) \frac{1}{2 \epsilon} \operatorname{sgn}(Z-W) \\
& \times\left\{k^{\alpha}(W), J_{[\mathbf{q}]}\right\} \Phi^{i}\left(\frac{\mathbf{S}(Z)}{\epsilon}+\boldsymbol{\theta}+\Delta \boldsymbol{\theta}, \mathbf{U}(Z)\right) \frac{\mathrm{d}^{m} \theta}{(2 \pi)^{m}} \mathrm{~d} Z \mathrm{~d} W \\
+\int Q_{i}\left(\frac{\mathbf{S}(Z)}{\epsilon}\right. & +\boldsymbol{\theta}, Z) C_{(0)}^{i \mu}\left(\boldsymbol{\Phi}\left(\frac{\mathbf{S}(Z)}{\epsilon}+\boldsymbol{\theta}+\Delta \boldsymbol{\theta}, \mathbf{U}(Z)\right), k^{\gamma} \mathbf{\Phi}_{\theta^{\gamma}}\left(\frac{\mathbf{S}(Z)}{\epsilon}+\boldsymbol{\theta}+\Delta \boldsymbol{\theta}, \mathbf{U}(Z)\right), \ldots\right) \\
& \times q_{\mu}(Z) \frac{\mathrm{d}^{m} \theta}{(2 \pi)^{m}} \mathrm{~d} Z+O(\epsilon) .
\end{aligned}
$$

Because of the invariance under translations (3.8) the value of the bracket $\left\{k^{\alpha}(W), J_{[\mathbf{q}]}\right\}$ on the functions (3.13) is equal to its value on $\mathcal{K}$

$$
\left\{k^{\alpha}(W), J_{[\mathbf{q}]}\right\}=\epsilon\left(\omega^{\alpha \nu}(W) q_{\nu}(W)\right)_{W}+O\left(\epsilon^{2}\right) .
$$


Similarly, we have on the functions (3.13)

$$
\begin{aligned}
C_{(0)}^{i \mu} & \left(\boldsymbol{\Phi}\left(\frac{\mathbf{S}(Z)}{\epsilon}+\boldsymbol{\theta}+\Delta \boldsymbol{\theta}, \mathbf{U}(Z)\right), k^{\gamma} \mathbf{\Phi}_{\theta^{\gamma}}\left(\frac{\mathbf{S}(Z)}{\epsilon}+\boldsymbol{\theta}+\Delta \boldsymbol{\theta}, \mathbf{U}(Z)\right) \ldots\right) \\
& =\omega^{\alpha \mu}(Z) \Phi_{\theta^{\alpha}}^{i}\left(\frac{\mathbf{S}(Z)}{\epsilon}+\boldsymbol{\theta}+\Delta \boldsymbol{\theta}, \mathbf{U}(Z)\right) .
\end{aligned}
$$

We then obtain

$$
\left\{g_{[\mathbf{Q}]}, J_{[\mathbf{q}]}\right\}=\int q_{\mu}(Z) \omega^{\alpha \mu}(Z) \frac{\partial}{\partial \theta^{\alpha}}\left[Q_{i}(\boldsymbol{\theta}, Z) \Phi^{i}(\boldsymbol{\theta}+\Delta \boldsymbol{\theta}, \mathbf{U}(Z))\right] \frac{\mathrm{d}^{m} \theta}{(2 \pi)^{m}} \mathrm{~d} Z+O(\epsilon)=O(\epsilon) .
$$

As a consequence, we can write

$$
\begin{aligned}
& \left.\iint_{0}^{2 \pi} \cdots \int_{0}^{2 \pi} \frac{\delta\left\{g_{[\mathbf{Q}]}, J_{[\mathbf{q}]}\right\}}{\delta g^{k}(\boldsymbol{\theta}, W)}\right|_{\mathcal{K}[0]} \Phi_{\theta^{\alpha}}^{k}\left(\frac{\mathbf{S}(W)}{\epsilon}+\boldsymbol{\theta}, \mathbf{U}(W)\right) \frac{\mathrm{d}^{m} \theta}{(2 \pi)^{m}} \mathrm{~d} W \equiv 0, \\
& \alpha=1, \ldots, m
\end{aligned}
$$

for the main part of $\delta\left\{g_{[\mathbf{Q}]}, J_{[\mathbf{q}]}\right\} / \delta g^{k}(\boldsymbol{\theta}, W)$ on $\mathcal{K}$.

Using again the fact that the relation (3.14) contains arbitrary functions $q_{\mu}(W)$, appearing in the integrand expression in the form of local factors, we can rewrite (3.14) in a stronger form

$$
\begin{aligned}
& \left.\int_{0}^{2 \pi} \cdots \int_{0}^{2 \pi} \frac{\delta\left\{g_{[\mathbf{Q}]}, J_{[\mathbf{q}]}\right\}}{\delta g^{k}(\boldsymbol{\theta}, W)}\right|_{\mathcal{K}[0]} \Phi_{\theta^{\alpha}}^{k}\left(\frac{\mathbf{S}(W)}{\epsilon}+\boldsymbol{\theta}, \mathbf{U}(W)\right) \frac{\mathrm{d}^{m} \theta}{(2 \pi)^{m}} \equiv 0, \\
& \forall W, \quad \alpha=1, \ldots, m .
\end{aligned}
$$

We now turn back to the Jacobi identity (3.5) for the functionals $g_{[\mathbf{Q}]}, J_{[\mathbf{q}]}$, and $J_{[\mathbf{p}]}$. It is not difficult to see that after the restriction on $\mathcal{K}$ the leading term $(\sim \epsilon)$ of the relation (3.5) can be written as

$$
\begin{aligned}
& \left.\left.\int\left\{g_{[\mathbf{Q}]}, g^{k}(\boldsymbol{\theta}, W)\right\}\right|_{\mathcal{K}[0]} \frac{\delta\left\{J_{[\mathbf{q}]}, J_{[\mathbf{p}]}\right\}}{\delta g^{k}(\boldsymbol{\theta}, W)}\right|_{\mathcal{K}[1]} \frac{\mathrm{d}^{m} \theta}{(2 \pi)^{m}} \mathrm{~d} W \\
& \quad+\left.\left.\int\left\{J_{[\mathbf{p}]}, g^{k}(\boldsymbol{\theta}, W)\right\}\right|_{\mathcal{K}[1]} \frac{\delta\left\{g_{[\mathbf{Q}]}, J_{[\mathbf{q}]}\right\}}{\delta g^{k}(\boldsymbol{\theta}, W)}\right|_{\mathcal{K}[0]} \frac{\mathrm{d}^{m} \theta}{(2 \pi)^{m}} \mathrm{~d} W \\
& \quad-\left.\left.\int\left\{J_{[\mathbf{q}]}, g^{k}(\boldsymbol{\theta}, W)\right\}\right|_{\mathcal{K}[1]} \frac{\delta\left\{g_{[\mathbf{Q}]}, J_{[\mathbf{p}]}\right\}}{\delta g^{k}(\boldsymbol{\theta}, W)}\right|_{\mathcal{K}[0]} \frac{\mathrm{d}^{m} \theta}{(2 \pi)^{m}} \mathrm{~d} W \equiv 0 .
\end{aligned}
$$

The above identity can again be written in a stronger form. Namely, making the change $\tilde{Q}_{i}(\boldsymbol{\theta}, W) \rightarrow \tilde{Q}_{i}(\boldsymbol{\theta}, W) \mu_{i}(W)$, we get the corresponding change, $Q_{i}(\boldsymbol{\theta}, W) \rightarrow Q_{i}(\boldsymbol{\theta}, W) \mu_{i}(W)$, where $\mu_{i}(W)$ are arbitrary smooth functions of $W$. By virtue of (3.4), (3.10), and (3.12) it is easy to see then that the integrands are smooth functions of $\boldsymbol{\theta}$ and $W$, containing $\mu_{i}(W)$ in the form of local factors. By the arbitrariness of $\mu_{i}(W)$, we can omit the integration over $W$ in the above integrals and write for every $W$

$$
\begin{aligned}
\int_{0}^{2 \pi} & \left.\left.\cdots \int_{0}^{2 \pi}\left\{g_{[\mathbf{Q}]}, g^{k}(\boldsymbol{\theta}, W)\right\}\right|_{\mathcal{K}[0]} \frac{\delta\left\{J_{[\mathbf{q}]}, J_{[\mathbf{p}]}\right\}}{\delta g^{k}(\boldsymbol{\theta}, W)}\right|_{\mathcal{K}[1]} \frac{\mathrm{d}^{m} \theta}{(2 \pi)^{m}} \\
& +\left.\left.\int_{0}^{2 \pi} \cdots \int_{0}^{2 \pi}\left\{J_{[\mathbf{p}]}, g^{k}(\boldsymbol{\theta}, W)\right\}\right|_{\mathcal{K}[1]} \frac{\delta\left\{g_{[\mathbf{Q}]}, J_{[\mathbf{q}]}\right\}}{\delta g^{k}(\boldsymbol{\theta}, W)}\right|_{\mathcal{K}[0]} \frac{\mathrm{d}^{m} \theta}{(2 \pi)^{m}} \\
& -\left.\left.\int_{0}^{2 \pi} \cdots \int_{0}^{2 \pi}\left\{J_{[\mathbf{q}]}, g^{k}(\boldsymbol{\theta}, W)\right\}\right|_{\mathcal{K}[1]} \frac{\delta\left\{g_{[\mathbf{Q}]}, J_{[\mathbf{p}]}\right\}}{\delta g^{k}(\boldsymbol{\theta}, W)}\right|_{\mathcal{K}[0]} \frac{\mathrm{d}^{m} \theta}{(2 \pi)^{m}} \equiv 0 .
\end{aligned}
$$


Finally, using the relations (2.61) and (3.15), we can write the above identity as

$$
\begin{aligned}
\int_{0}^{2 \pi} & \left.\left.\cdots \int_{0}^{2 \pi}\left\{g_{[\mathbf{Q}]}, g^{k}(\boldsymbol{\theta}, W)\right\}\right|_{\mathcal{K}[0]} \frac{\delta\left\{J_{[\mathbf{q}]}, J_{[\mathbf{p}]}\right\}}{\delta g^{k}(\boldsymbol{\theta}, W)}\right|_{\mathcal{K}[1]} \frac{\mathrm{d}^{m} \theta}{(2 \pi)^{m}} \\
& -\left.\int_{0}^{2 \pi} \cdots \int_{0}^{2 \pi} A_{[1][\mathbf{p}]}^{k}\left(\frac{\mathbf{S}(W)}{\epsilon}+\boldsymbol{\theta}, W\right) \frac{\delta\left\{g_{[\mathbf{Q}]}, J_{[\mathbf{q}]}\right\}}{\delta g^{k}(\boldsymbol{\theta}, W)}\right|_{\mathcal{K}[0]} \frac{\mathrm{d}^{m} \theta}{(2 \pi)^{m}} \\
& +\left.\int_{0}^{2 \pi} \cdots \int_{0}^{2 \pi} A_{[1][\mathbf{q}]}^{k}\left(\frac{\mathbf{S}(W)}{\epsilon}+\boldsymbol{\theta}, W\right) \frac{\delta\left\{g_{[\mathbf{Q}]}, J_{[\mathbf{p}]}\right\}}{\delta g^{k}(\boldsymbol{\theta}, W)}\right|_{\mathcal{K}[0]} \frac{\mathrm{d}^{m} \theta}{(2 \pi)^{m}} \equiv 0
\end{aligned}
$$

where the functions $A_{[1][\mathbf{q}]}^{k}(\boldsymbol{\theta}, W)$ are introduced by formula (2.64).

Now assume that the values $A_{[1][\mathbf{q}]}^{k}(\boldsymbol{\theta}, W)$ for $\mathbf{U}(W) \in \mathcal{S}$, according to (3.1), can be represented in the form

$$
A_{[1][\mathbf{q}]}^{k}(\boldsymbol{\theta}, W)=-\hat{B}_{[0]}^{k j}(W) B_{j[\mathbf{q}](1)}(\boldsymbol{\theta}, W)
$$

with some smooth in $\boldsymbol{\theta}, 2 \pi$-periodic in each $\theta^{\alpha}$ functions $B_{j[\mathbf{q}](1)}(\boldsymbol{\theta}, W)$.

We can then write for $\mathbf{U}(W) \in \mathcal{S}$

$$
\begin{aligned}
\int_{0}^{2 \pi} & \left.\left.\cdots \int_{0}^{2 \pi}\left\{g_{[\mathbf{Q}]}, g^{k}(\boldsymbol{\theta}, W)\right\}\right|_{\mathcal{K}[0]} \frac{\delta\left\{J_{[\mathbf{q}]}, J_{[\mathbf{p}]}\right\}}{\delta g^{k}(\boldsymbol{\theta}, W)}\right|_{\mathcal{K}[1]} \frac{\mathrm{d}^{m} \theta}{(2 \pi)^{m}} \\
& +\left.\int_{0}^{2 \pi} \cdots \int_{0}^{2 \pi}\left[\hat{B}_{[0][\mathbf{S}]}^{k j}(W) B_{j[\mathbf{p}](1)}\left(\frac{\mathbf{S}(W)}{\epsilon}+\boldsymbol{\theta}, W\right)\right] \frac{\delta\left\{g_{[\mathbf{Q}]}, J_{[\mathbf{q}]}\right\}}{\delta g^{k}(\boldsymbol{\theta}, W)}\right|_{\mathcal{K}[0]} \frac{\mathrm{d}^{m} \theta}{(2 \pi)^{m}} \\
& -\left.\int_{0}^{2 \pi} \cdots \int_{0}^{2 \pi}\left[\hat{B}_{[0][\mathbf{S}]}^{k j}(W) B_{j[\mathbf{q}](1)}\left(\frac{\mathbf{S}(W)}{\epsilon}+\boldsymbol{\theta}, W\right)\right] \frac{\delta\left\{g_{[\mathbf{Q}]}, J_{[\mathbf{p}]}\right\}}{\delta g^{k}(\boldsymbol{\theta}, W)}\right|_{\mathcal{K}[0]} \frac{\mathrm{d}^{m} \theta}{(2 \pi)^{m}} \equiv 0
\end{aligned}
$$

where

$$
\begin{aligned}
\hat{B}_{[0][\mathbf{S}]}^{i j}(W) \equiv & \sum_{s \geq 0} B_{(s)}^{i j}\left(\boldsymbol{\Phi}\left(\frac{\mathbf{S}(W)}{\epsilon}+\boldsymbol{\theta}, \mathbf{U}(W)\right), k^{\gamma}(W) \boldsymbol{\Phi}_{\theta^{\gamma}}\left(\frac{\mathbf{S}(W)}{\epsilon}+\boldsymbol{\theta}, \mathbf{U}(W)\right), \ldots\right) \\
& \times k^{\alpha_{1}}(W) \cdots k^{\alpha_{s}}(W) \frac{\partial^{s}}{\partial \theta^{\alpha_{1}} \cdots \partial \theta^{\alpha_{s}}} .
\end{aligned}
$$

Using the expression (2.54) for the bracket of constraints on $\mathcal{K}$, as well as the relations (2.42), (3.4), (3.10), we can obtain also the following relation

$$
\begin{aligned}
\int_{0}^{2 \pi} & \left.\left.\cdots \int_{0}^{2 \pi}\left\{g_{[\mathbf{Q}]}, g^{k}(\boldsymbol{\theta}, W)\right\}\right|_{\mathcal{K}[0]} \frac{\delta\left\{J_{[\mathbf{q}]}, J_{[\mathbf{p}]}\right\}}{\delta g^{k}(\boldsymbol{\theta}, W)}\right|_{\mathcal{K}[1]} \frac{\mathrm{d}^{m} \theta}{(2 \pi)^{m}} \\
& =-\left.\int_{0}^{2 \pi} \cdots \int_{0}^{2 \pi}\left[\hat{B}_{[0][\mathbf{S}]}^{k j}(W) Q_{j}\left(\frac{\mathbf{S}(W)}{\epsilon}+\boldsymbol{\theta}, W\right)\right] \frac{\delta\left\{J_{[\mathbf{q}]}, J_{[\mathbf{p}]}\right\}}{\delta g^{k}(\boldsymbol{\theta}, W)}\right|_{\mathcal{K}[1]} \frac{\mathrm{d}^{m} \theta}{(2 \pi)^{m}} .
\end{aligned}
$$

Expanding also the remaining terms of the identity (3.17) according to (3.12), we get for $\mathbf{U}(W) \in \mathcal{S}$

$$
\begin{aligned}
\int_{0}^{2 \pi} & \left.\cdots \int_{0}^{2 \pi}\left[\hat{B}_{[0][\mathbf{S}]}^{k j}(W) Q_{j}\left(\frac{\mathbf{S}(W)}{\epsilon}+\boldsymbol{\theta}, W\right)\right] \frac{\delta\left\{J_{[\mathbf{q}]}, J_{[\mathbf{p}]}\right\}}{\delta g^{k}(\boldsymbol{\theta}, W)}\right|_{\mathcal{K}[1]} \frac{\mathrm{d}^{m} \theta}{(2 \pi)^{m}} \\
& -\int_{0}^{2 \pi} \cdots \int_{0}^{2 \pi} \frac{\mathrm{d}^{m} \theta}{(2 \pi)^{m}}\left[\hat{B}_{[0][\mathbf{S}]}^{k j}(W) B_{j[\mathbf{p}](1)}\left(\frac{\mathbf{S}(W)}{\epsilon}+\boldsymbol{\theta}, W\right)\right] \\
& \times\left[\sum_{l \geq 0}(-1)^{l} k^{\alpha_{1}}(W) \cdots k^{\alpha_{l}}(W) \frac{\partial^{l}}{\partial \theta^{\alpha_{1}} \cdots \partial \theta^{\alpha_{l}}}\right.
\end{aligned}
$$




$$
\begin{gathered}
\times\left[\left.Q_{i}\left(\frac{\mathbf{S}(W)}{\epsilon}+\boldsymbol{\theta}, W\right) S_{k(l)}^{i \mu}(\boldsymbol{\varphi}(\boldsymbol{\theta}, W), \ldots)\right|_{\mathcal{K}[0]}\right] q_{\mu}(W) \\
\left.+\omega^{\alpha \mu}(W) q_{\mu}(W) Q_{k, \theta^{\alpha}}\left(\frac{\mathbf{S}(W)}{\epsilon}+\boldsymbol{\theta}, W\right)\right] \\
+\int_{0}^{2 \pi} \cdots \int_{0}^{2 \pi} \frac{\mathrm{d}^{m} \theta}{(2 \pi)^{m}}\left[\hat{B}_{[0][\mathbf{S}]}^{k j}(W) B_{j[\mathbf{q}](1)}\left(\frac{\mathbf{S}(W)}{\epsilon}+\boldsymbol{\theta}, W\right)\right] \\
\times\left[\sum_{l \geq 0}(-1)^{l} k^{\alpha_{1}}(W) \cdots k^{\alpha_{l}}(W) \frac{\partial^{l}}{\partial \theta^{\alpha_{1}} \cdots \partial \theta^{\alpha_{l}}}\right. \\
\times\left[\left.Q_{i}\left(\frac{\mathbf{S}(W)}{\epsilon}+\boldsymbol{\theta}, W\right) S_{k(l)}^{i \mu}(\boldsymbol{\varphi}(\boldsymbol{\theta}, W), \ldots)\right|_{\mathcal{K}[0]}\right] p_{\mu}(W) \\
\left.\quad+\omega^{\alpha \mu}(W) p_{\mu}(W) Q_{k, \theta^{\alpha}}\left(\frac{\mathbf{S}(W)}{\epsilon}+\boldsymbol{\theta}, W\right)\right] \equiv 0 .
\end{gathered}
$$

Consider now the Jacobi identity of the form

$$
\left\{g_{[\mathbf{P}]},\left\{g_{[\mathbf{Q}]}, J_{[\mathbf{q}]}\right\}\right\}+\left\{g_{[\mathbf{Q}]},\left\{J_{[\mathbf{q}]}, g_{[\mathbf{P}]}\right\}\right\}+\left\{J_{[\mathbf{q}]},\left\{g_{[\mathbf{P}]}, g_{[\mathbf{Q}]}\right\}\right\} \equiv 0
$$

for arbitrary fixed functions $\mathbf{q}(X)$, and the functionals $\mathbf{P}(\boldsymbol{\theta}, X)$ and $\mathbf{Q}(\boldsymbol{\theta}, X)$ defined as before with the aid of arbitrary functions $\tilde{\mathbf{P}}(\boldsymbol{\theta}, X), \tilde{\mathbf{Q}}(\boldsymbol{\theta}, X)$.

According to the relations

$$
\left.\left\{g_{[\mathbf{Q}, \mathbf{P}]}, U^{\mu}(W)\right\}\right|_{\mathcal{K}}=O(\epsilon),\left.\quad\left\{J_{[\mathbf{q}]}, g^{k}(\boldsymbol{\theta}, W)\right\}\right|_{\mathcal{K}}=O(\epsilon),\left.\quad\left\{J_{[\mathbf{q}]}, U^{\mu}(W)\right\}\right|_{\mathcal{K}}=O(\epsilon)
$$

it's not difficult to see that after the restriction on $\mathcal{K}$ the major term (in $\epsilon$ ) of (3.19) will be written as

$$
\begin{aligned}
& \left.\left.\int\left\{g_{[\mathbf{P}]}, g^{k}(\boldsymbol{\theta}, W)\right\}\right|_{\mathcal{K}[0]} \frac{\delta\left\{g_{[\mathbf{Q}]}, J_{[\mathbf{q}]}\right\}}{\delta g^{k}(\boldsymbol{\theta}, W)}\right|_{\mathcal{K}[0]} \frac{\mathrm{d}^{m} \theta}{(2 \pi)^{m}} \mathrm{~d} W \\
& \quad-\left.\left.\int\left\{g_{[\mathbf{Q}]}, g^{k}(\boldsymbol{\theta}, W)\right\}\right|_{\mathcal{K}[0]} \frac{\delta\left\{g_{[\mathbf{P}]}, J_{[\mathbf{q}]}\right\}}{\delta g^{k}(\boldsymbol{\theta}, W)}\right|_{\mathcal{K}[0]} \frac{\mathrm{d}^{m} \theta}{(2 \pi)^{m}} \mathrm{~d} W \equiv 0 .
\end{aligned}
$$

Again recalling that $q_{\nu}(W)$ are arbitrary functions of $W$ appearing in the integrand in the form of local factors, we can write the above relation in a stronger form. That is, for every $W$

$$
\begin{aligned}
\int_{0}^{2 \pi} & \left.\left.\cdots \int_{0}^{2 \pi}\left\{g_{[\mathbf{P}]}, g^{k}(\boldsymbol{\theta}, W)\right\}\right|_{\mathcal{K}[0]} \frac{\delta\left\{g_{[\mathbf{Q}]}, J_{[\mathbf{q}]}\right\}}{\delta g^{k}(\boldsymbol{\theta}, W)}\right|_{\mathcal{K}[0]} \frac{\mathrm{d}^{m} \theta}{(2 \pi)^{m}} \\
& -\left.\left.\int_{0}^{2 \pi} \cdots \int_{0}^{2 \pi}\left\{g_{[\mathbf{Q}]}, g^{k}(\boldsymbol{\theta}, W)\right\}\right|_{\mathcal{K}[0]} \frac{\delta\left\{g_{[\mathbf{P}]}, J_{[\mathbf{q}]}\right\}}{\delta g^{k}(\boldsymbol{\theta}, W)}\right|_{\mathcal{K}[0]} \frac{\mathrm{d}^{m} \theta}{(2 \pi)^{m}} \equiv 0 .
\end{aligned}
$$

As well as in the case of the identity (3.18), using the relations (2.54), (2.42), (3.4), (3.15), we can write the identity (3.20) in the form

$$
\begin{aligned}
\int_{0}^{2 \pi} & \cdots \int_{0}^{2 \pi} \frac{\mathrm{d}^{m} \theta}{(2 \pi)^{m}}\left[\hat{B}_{[0][\mathbf{S}]}^{k j}(W) P_{j}\left(\frac{\mathbf{S}(W)}{\epsilon}+\boldsymbol{\theta}, W\right)\right] \\
\times & {\left[\sum_{l \geq 0}(-1)^{l} k^{\alpha_{1}}(W) \cdots k^{\alpha_{l}}(W) \frac{\partial^{l}}{\partial \theta^{\alpha_{1}} \cdots \partial \theta^{\alpha_{l}}}\right.} \\
& \times\left[\left.Q_{i}\left(\frac{\mathbf{S}(W)}{\epsilon}+\boldsymbol{\theta}, W\right) S_{k(l)}^{i \mu}(\boldsymbol{\varphi}(\boldsymbol{\theta}, W), \ldots)\right|_{\mathcal{K}[0]}\right] q_{\mu}(W)
\end{aligned}
$$




$$
\begin{gathered}
\left.+\omega^{\alpha \mu}(W) q_{\mu}(W) Q_{k, \theta^{\alpha}}\left(\frac{\mathbf{S}(W)}{\epsilon}+\boldsymbol{\theta}, W\right)\right] \\
-\int_{0}^{2 \pi} \cdots \int_{0}^{2 \pi} \frac{\mathrm{d}^{m} \theta}{(2 \pi)^{m}}\left[\hat{B}_{[0][\mathbf{S}]}^{k j}(W) Q_{j}\left(\frac{\mathbf{S}(W)}{\epsilon}+\boldsymbol{\theta}, W\right)\right] \\
\times\left[\sum_{l \geq 0}(-1)^{l} k^{\alpha_{1}}(W) \cdots k^{\alpha_{l}}(W) \frac{\partial^{l}}{\partial \theta^{\alpha_{1}} \cdots \partial \theta^{\alpha_{l}}}\right. \\
\times\left[\left.P_{i}\left(\frac{\mathbf{S}(W)}{\epsilon}+\boldsymbol{\theta}, W\right) S_{k(l)}^{i \mu}(\boldsymbol{\varphi}(\boldsymbol{\theta}, W), \ldots)\right|_{\mathcal{K}[0]}\right] q_{\mu}(W) \\
\left.\quad+\omega^{\alpha \mu}(W) q_{\mu}(W) P_{k, \theta^{\alpha}}\left(\frac{\mathbf{S}(W)}{\epsilon}+\boldsymbol{\theta}, W\right)\right] \equiv 0 .
\end{gathered}
$$

Note now that the values of $\mathbf{Q}(\boldsymbol{\theta}, X)$ and $\mathbf{P}(\boldsymbol{\theta}, X)$ are arbitrary $2 \pi$-periodic functions of $\boldsymbol{\theta}$, satisfying the conditions (3.4). In particular, we can put in (3.21) at $\mathbf{U}(W) \in \mathcal{S}$

$$
\mathbf{P}(\boldsymbol{\theta}, W)=\mathbf{B}_{[\mathbf{p}](1)}(\boldsymbol{\theta}, W) \quad \text { or } \quad \mathbf{P}(\boldsymbol{\theta}, W)=\mathbf{B}_{[\mathbf{q}](1)}(\boldsymbol{\theta}, W) .
$$

By analogy with (3.12) we introduce for convenience the notation for $\mathbf{U}(W) \in \mathcal{S}$

$$
\begin{aligned}
\left.\frac{\delta\left\{\left.g_{\left[\epsilon \mathbf{B}_{[\mathbf{p}](1)}, J_{[\mathbf{q}]}\right\}}\right|_{g^{k}(\boldsymbol{\theta}, W)} \equiv\right.}{\equiv}\right|_{\mathcal{K}[1]}(-1)^{l} k^{\alpha_{1}}(W) \cdots k^{\alpha_{l}}(W) \frac{\partial^{l}}{\partial \theta^{\alpha_{1}} \cdots \partial \theta^{\alpha_{l}}} \\
\times\left[\left.B_{i[\mathbf{p}](1)}\left(\frac{\mathbf{S}(W)}{\epsilon}+\boldsymbol{\theta}, W\right) S_{k(l)}^{i \mu}(\boldsymbol{\varphi}(\boldsymbol{\theta}, W), \ldots)\right|_{\mathcal{K}[0]}\right] q_{\mu}(W) \\
\left.+\omega^{\alpha \mu}(W) q_{\mu}(W) B_{k[\mathbf{p}](1), \theta^{\alpha}}\left(\frac{\mathbf{S}(W)}{\epsilon}+\boldsymbol{\theta}, W\right)\right]
\end{aligned}
$$

for arbitrary smooth functions $\mathbf{q}(X), \mathbf{p}(X)$.

Note that the functional $g_{\left[\in \mathbf{B}_{[\mathbf{p}](1)}\right]}$ is not defined on the whole functional space, so the relation (3.23) plays just a role of a formal notation for $\mathbf{U}(W) \in \mathcal{S}$.

Using now (3.21) for the functions (3.22), we can rewrite (3.18) in the form

$$
\begin{aligned}
\int_{0}^{2 \pi} & \cdots \int_{0}^{2 \pi} \frac{\mathrm{d}^{m} \theta}{(2 \pi)^{m}}\left[\hat{B}_{[0][\mathbf{S}]}^{k j}(W) Q_{j}\left(\frac{\mathbf{S}(W)}{\epsilon}+\boldsymbol{\theta}, W\right)\right] \\
& \times\left(\left.\frac{\delta\left\{J_{[\mathbf{q}]}, J_{[\mathbf{p}]}\right\}}{\delta g^{k}(\boldsymbol{\theta}, W)}\right|_{\mathcal{K}[1]}-\left.\frac{\delta\left\{g_{\left[\epsilon \mathbf{B}_{[\mathbf{p}](1)}\right]}, J_{[\mathbf{q}]}\right\}}{\delta g^{k}(\boldsymbol{\theta}, W)}\right|_{\mathcal{K}[1]}+\left.\frac{\delta\left\{g_{\left[\epsilon \mathbf{B}_{[\mathbf{q}](1)}\right]}, J_{[\mathbf{p}]}\right\}}{\delta g^{k}(\boldsymbol{\theta}, W)}\right|_{\mathcal{K}[1]}\right) \equiv 0
\end{aligned}
$$

provided that $\mathbf{U}(W) \in \mathcal{S}$.

Using the skew-symmetry of the Hamiltonian operator $\hat{B}_{[0][\mathbf{S}]}^{k j}(W)$ on $\Lambda$ we can then write

$$
\begin{aligned}
& \int_{0}^{2 \pi} \cdots \int_{0}^{2 \pi} \frac{\mathrm{d}^{m} \theta}{(2 \pi)^{m}} Q_{j}\left(\frac{\mathbf{S}(W)}{\epsilon}+\boldsymbol{\theta}, W\right) \\
& \times\left[\hat{B}_{[0][\mathbf{S}]}^{j k}(W)\left(\left.\frac{\delta\left\{J_{[\mathbf{q}]}, J_{[\mathbf{p}]}\right\}}{\delta g^{k}(\boldsymbol{\theta}, W)}\right|_{\mathcal{K}[1]}-\left.\frac{\delta\left\{g_{\left[\epsilon \mathbf{B}_{[\mathbf{p}](1)}\right.}, J_{[\mathbf{q}]}\right\}}{\delta g^{k}(\boldsymbol{\theta}, W)}\right|_{\mathcal{K}[1]}+\left.\frac{\delta\left\{g_{\left[\epsilon \mathbf{B}_{[\mathbf{q}](1)}\right]}, J_{[\mathbf{p}]}\right\}}{\delta g^{k}(\boldsymbol{\theta}, W)}\right|_{\mathcal{K}[1]}\right)\right] \equiv 0 .
\end{aligned}
$$

The values $Q_{j}(\boldsymbol{\theta}, W)$ are arbitrary smooth $2 \pi$-periodic functions of $\boldsymbol{\theta}$ with the only restriction (3.4). We know also that the values in the brackets are smooth $2 \pi$-periodic in each $\theta^{\alpha}$ 
functions of $\boldsymbol{\theta}$ for $\mathbf{U}(W) \in \mathcal{S}$. As a consequence, we can write for $\mathbf{U}(W) \in \mathcal{S}$

$$
\begin{aligned}
\hat{B}_{[0][\mathbf{S}]}^{j k}(W)\left(\left.\frac{\delta\left\{J_{[\mathbf{q}]}, J_{[\mathbf{p}]}\right\}}{\delta g^{k}(\boldsymbol{\theta}, W)}\right|_{\mathcal{K}[1]}-\left.\frac{\delta\left\{g_{\left[\epsilon \mathbf{B}_{[\mathbf{p}](1)}\right]}, J_{[\mathbf{q}]}\right\}}{\delta g^{k}(\boldsymbol{\theta}, W)}\right|_{\mathcal{K}[1]}+\left.\frac{\delta\left\{g_{\left[\epsilon \mathbf{B}_{[\mathbf{q q}](1)}\right]}, J_{[\mathbf{p}]}\right\}}{\delta g^{k}(\boldsymbol{\theta}, W)}\right|_{\mathcal{K}[1]}\right) \\
\equiv \sum_{\alpha=1}^{m} a^{\alpha}\left(\mathbf{U}(W), \mathbf{U}_{W}(W)\right) \Phi_{\theta^{\alpha}}^{j}\left(\frac{\mathbf{S}(W)}{\epsilon}+\boldsymbol{\theta}, \mathbf{U}(W)\right)
\end{aligned}
$$

with some coefficients $a^{\alpha}\left(\mathbf{U}(W), \mathbf{U}_{W}(W)\right)$.

The values in parentheses are smooth $2 \pi$-periodic functions of $\boldsymbol{\theta}$ at $\mathbf{U}(W) \in \mathcal{S}$. At the same time the trajectories of the vector field $\left(k^{1}(W), \ldots, k^{m}(W)\right)$ are completely irrational windings of the torus $\mathbb{T}^{m}$. We can therefore say in the case of a regular Hamiltonian family $\Lambda$, that up to a linear combination of the regular eigenvectors $\mathbf{v}_{[\mathbf{U}(W)]}^{(l)}(\mathbf{S}(W) / \epsilon+\boldsymbol{\theta})$ of $\hat{B}_{[0][\mathbf{S}]}^{j k}(W)=$ $\hat{B}_{[0][\mathbf{S}]}^{j k}(\mathbf{U}(W))$, corresponding to zero eigenvalues, the value in parentheses is a linear combination of the variational derivatives (2.7), generating linear shifts of the phases on $\Lambda$. For complete Hamiltonian set of the integrals $\left(I^{1}, \ldots, I^{N}\right)$ we can then write according to (2.8) and (3.3)

$$
\begin{gathered}
\left.\frac{\delta\left\{J_{[\mathbf{q}]}, J_{[\mathbf{p}]}\right\}}{\delta g^{k}(\boldsymbol{\theta}, W)}\right|_{\mathcal{K}[1]}-\left.\frac{\delta\left\{g_{\left[\epsilon \mathbf{B}_{[\mathbf{p}](1)},\right.}, J_{[\mathbf{q}]}\right\}}{\delta g^{k}(\boldsymbol{\theta}, W)}\right|_{\mathcal{K}[1]}+\left.\frac{\delta\left\{g_{\left[\epsilon \mathbf{B}_{[\mathbf{q q}](1)}\right]}, J_{[\mathbf{p}]}\right\}}{\delta g^{k}(\boldsymbol{\theta}, W)}\right|_{\mathcal{K}[1]} \\
\equiv \sum_{q} b_{q}\left(\mathbf{U}(W), \mathbf{U}_{W}(W)\right) \kappa_{k[\mathbf{U}(W)]}^{(q)}\left(\frac{\mathbf{S}(W)}{\epsilon}+\boldsymbol{\theta}\right)
\end{gathered}
$$

with some coefficients $b_{q}\left(\mathbf{U}(W), \mathbf{U}_{W}(W)\right)$ at $\mathbf{U}(W) \in \mathcal{S}$.

Consider the Jacobi identity of the form

$$
\left\{\left\{J_{[\mathbf{q}]}, J_{[\mathbf{p}]}\right\}, J_{[\mathbf{r}]}\right\}+\left\{\left\{J_{[\mathbf{p}]}, J_{[\mathbf{r}]}\right\}, J_{[\mathbf{q}]}\right\}+\left\{\left\{J_{[\mathbf{r}]}, J_{[\mathbf{q}]}\right\}, J_{[\mathbf{p}]}\right\} \equiv 0
$$

with arbitrary smooth functions $\mathbf{q}(X), \mathbf{p}(X), \mathbf{r}(X)$.

In the main $\left(\sim \epsilon^{2}\right)$ order on $\mathcal{K}$ given identity leads to the relations

$$
\begin{aligned}
& \left.\left.\int \frac{\delta\left\{J_{[\mathbf{q}]}, J_{[\mathbf{p}]}\right\}}{\delta U^{\gamma}(W)}\right|_{\mathcal{K}[1]}\left\{U^{\gamma}(W), J_{[\mathbf{r}]}\right\}\right|_{\mathcal{K}[1]} \mathrm{d} W+\text { c.p. } \\
& \quad+\left.\left.\iint_{0}^{2 \pi} \cdots \int_{0}^{2 \pi} \frac{\delta\left\{J_{[\mathbf{q}]}, J_{[\mathbf{p}]}\right\}}{\delta g^{k}(\boldsymbol{\theta}, W)}\right|_{\mathcal{K}[1]}\left\{g^{k}(\boldsymbol{\theta}, W), J_{[\mathbf{r}]}\right\}\right|_{\mathcal{K}[1]} \frac{\mathrm{d}^{m} \theta}{(2 \pi)^{m}} \mathrm{~d} W+\text { c.p. } \equiv 0 .
\end{aligned}
$$

Again, using the relations (2.61), (2.64) and (3.10), we can replace the identity (3.25) by the following relation

$$
\begin{aligned}
& \left.\left.\int \frac{\delta\left\{J_{[\mathbf{q}]}, J_{[\mathbf{p}]}\right\}}{\delta U^{\gamma}(W)}\right|_{\mathcal{K}[1]}\left\{U^{\gamma}(W), J_{[\mathbf{r}]}\right\}\right|_{\mathcal{K}[1]} \mathrm{d} W+\text { c.p. } \\
& \quad+\left.\iint_{0}^{2 \pi} \cdots \int_{0}^{2 \pi} \frac{\delta\left\{J_{[\mathbf{q}]}, J_{[\mathbf{p}]}\right\}}{\delta g^{k}(\boldsymbol{\theta}, W)}\right|_{\mathcal{K}[1]} A_{[1][\mathbf{r}]}^{k}\left(\frac{\mathbf{S}(W)}{\epsilon}+\boldsymbol{\theta}, W\right) \frac{\mathrm{d}^{m} \theta}{(2 \pi)^{m}} \mathrm{~d} W+\text { c.p. } \equiv 0 .
\end{aligned}
$$

Using relations (2.71) and representations (3.16) and (3.24), we can write for $\mathbf{U}(W) \in \mathcal{S}$

$$
\begin{aligned}
\int_{0}^{2 \pi} & \left.\cdots \int_{0}^{2 \pi} \frac{\delta\left\{J_{[\mathbf{q}]}, J_{[\mathbf{p}]}\right\}}{\delta g^{k}(\boldsymbol{\theta}, W)}\right|_{\mathcal{K}[1]} A_{[1][\mathbf{r}]}^{k}\left(\frac{\mathbf{S}(W)}{\epsilon}+\boldsymbol{\theta}, W\right) \frac{\mathrm{d}^{m} \theta}{(2 \pi)^{m}}+\text { c.p. } \\
& \equiv \int_{0}^{2 \pi} \cdots \int_{0}^{2 \pi}\left[\hat{B}_{[0][\mathbf{S}]}^{k j}(W) B_{j[\mathbf{r}](1)}\left(\frac{\mathbf{S}(W)}{\epsilon}+\boldsymbol{\theta}, W\right)\right]
\end{aligned}
$$




$$
\begin{aligned}
& \times\left[\left.\frac{\delta\left\{g_{\left[\epsilon \mathbf{B}_{[\mathbf{q}](1)}\right]}, J_{[\mathbf{p}]}\right\}}{\delta g^{k}(\boldsymbol{\theta}, W)}\right|_{\mathcal{K}[1]}-\left.\frac{\delta\left\{g_{\left[\epsilon \mathbf{B}_{[\mathbf{p}](1)]}, J_{[\mathbf{q}]}\right\}}\right.}{\delta g^{k}(\boldsymbol{\theta}, W)}\right|_{\mathcal{K}[1]}\right] \frac{\mathrm{d}^{m} \theta}{(2 \pi)^{m}} \\
+\int_{0}^{2 \pi} & \cdots \int_{0}^{2 \pi}\left[\hat{B}_{[0][\mathbf{S}]}^{k j}(W) B_{j[\mathbf{q}](1)}\left(\frac{\mathbf{S}(W)}{\epsilon}+\boldsymbol{\theta}, W\right)\right] \\
& \times\left[\left.\frac{\delta\left\{g_{\left[\epsilon \mathbf{B}_{[\mathbf{p}](1)}\right]}, J_{[\mathbf{r}]}\right\}}{\delta g^{k}(\boldsymbol{\theta}, W)}\right|_{\mathcal{K}[1]}-\left.\frac{\delta\left\{g_{\left[\epsilon \mathbf{B}_{[\mathbf{r}](1)}\right]}, J_{[\mathbf{p}]}\right\}}{\delta g^{k}(\boldsymbol{\theta}, W)}\right|_{\mathcal{K}[1]}\right] \frac{\mathrm{d}^{m} \theta}{(2 \pi)^{m}} \\
+\int_{0}^{2 \pi} & \cdots \int_{0}^{2 \pi}\left[\hat{B}_{[0][\mathbf{S}]}^{k j}(W) B_{j[\mathbf{p}](1)}\left(\frac{\mathbf{S}(W)}{\epsilon}+\boldsymbol{\theta}, W\right)\right] \\
& \times\left[\left.\frac{\delta\left\{g_{\left[\epsilon \mathbf{B}_{[\mathbf{r}](1)}\right]}, J_{[\mathbf{q}]}\right\}}{\delta g^{k}(\boldsymbol{\theta}, W)}\right|_{\mathcal{K}[1]}-\left.\frac{\delta\left\{g_{\left[\epsilon \mathbf{B}_{[\mathbf{q}](1)}\right]}, J_{[\mathbf{r}]}\right\}}{\delta g^{k}(\boldsymbol{\theta}, W)}\right|_{\mathcal{K}[1]}\right] \frac{\mathrm{d}^{m} \theta}{(2 \pi)^{m}} .
\end{aligned}
$$

Similarly to earlier arguments, substituting now in the identity (3.21)

$$
\mathbf{Q}(\boldsymbol{\theta}, W)=\mathbf{B}_{[\mathbf{r}](1)}(\boldsymbol{\theta}, W), \quad \mathbf{P}(\boldsymbol{\theta}, W)=\mathbf{B}_{[\mathbf{p}](1)}(\boldsymbol{\theta}, W)
$$

for $\mathbf{U}(W) \in \mathcal{S}$ we obtain the identity

$$
\begin{aligned}
& \left.\int_{0}^{2 \pi} \cdots \int_{0}^{2 \pi}\left[\hat{B}_{[0][\mathbf{S}]}^{k j}(W) B_{j[\mathbf{p}](1)}\left(\frac{\mathbf{S}(W)}{\epsilon}+\boldsymbol{\theta}, W\right)\right] \frac{\delta\left\{g_{\left[\epsilon \mathbf{B}_{[\mathbf{r}](1)}\right]}, J_{[\mathbf{q}]}\right\}}{\delta g^{k}(\boldsymbol{\theta}, W)}\right|_{\mathcal{K}[1]} \frac{\mathrm{d}^{m} \theta}{(2 \pi)^{m}} \\
& -\left.\int_{0}^{2 \pi} \cdots \int_{0}^{2 \pi}\left[\hat{B}_{[0][\mathbf{S}]}^{k j}(W) B_{j[\mathbf{r}](1)}\left(\frac{\mathbf{S}(W)}{\epsilon}+\boldsymbol{\theta}, W\right)\right] \frac{\delta\left\{g_{\left[\epsilon \mathbf{B}_{[\mathbf{p}](1)}\right]}, J_{[\mathbf{q}]}\right\}}{\delta g^{k}(\boldsymbol{\theta}, W)}\right|_{\mathcal{K}[1]} \frac{\mathrm{d}^{m} \theta}{(2 \pi)^{m}}=0 .
\end{aligned}
$$

Using the cyclic permutations of the functions $\mathbf{q}(X), \mathbf{p}(X)$, and $\mathbf{r}(X)$ in the identity (3.27), it's not difficult to see that the right-hand part of the relation (3.26) is identically equal to zero at $\mathbf{U}(W) \in \mathcal{S}$. It's not difficult to see also that the left-hand side of the expression (3.26) is a smooth regular function of the parameters $\mathbf{U}(W)$. Using the fact that the set $\mathcal{S}$ is everywhere dense in the parameter space $\mathbf{U}$, we can conclude that the left-hand side of equation (3.26) is identically equal to zero under the conditions of the theorem.

We have, therefore, that under the conditions of the theorem, the identity (3.25) implies the relation

$$
\left.\left.\int \frac{\delta\left\{J_{[\mathbf{q}]}, J_{[\mathbf{p}]}\right\}}{\delta U^{\gamma}(W)}\right|_{\mathcal{K}[1]}\left\{U^{\gamma}(W), J_{[\mathbf{r}]}\right\}\right|_{\mathcal{K}[1]} \mathrm{d} W+\text { c.p. } \equiv 0 .
$$

Using the relations

$$
\left.\frac{\delta\left\{J_{[\mathbf{q}]}, J_{[\mathbf{p}]}\right\}}{\delta U^{\gamma}(W)}\right|_{\mathcal{K}[1]}=\frac{\delta\left\{U_{[\mathbf{q}]}, U_{[\mathbf{p}]}\right\}_{\mathrm{DN}}}{\delta U^{\gamma}(W)},\left.\quad\left\{U^{\gamma}(W), J_{[\mathbf{r}]}\right\}\right|_{\mathcal{K}[1]}=\left\{U^{\gamma}(W), U_{[\mathbf{r}]}\right\}_{\mathrm{DN}}
$$

we obtain the Jacobi identity for the Dubrovin-Novikov bracket on the space of the functions $\mathbf{U}(X)$.

Remark 3.1. According to relations (2.47) the functionals

$$
K^{\alpha}=\int_{-\infty}^{+\infty} k^{\alpha}(\mathbf{U}(X)) \mathrm{d} X
$$

are annihilators of the Dubrovin-Novikov bracket (2.21) since we have $\left\{K^{\alpha}, U_{[\mathbf{q}]}\right\}=0$ for any functional $U_{[\mathbf{q}]}$. As a consequence, we can claim that the functions $k^{\alpha}(\mathbf{U})$ represent a part of the 
flat coordinates for the metric $g^{\nu \mu}(\mathbf{U})=\left\langle A_{1}^{\nu \mu}\right\rangle$ connected with the Dubrovin-Novikov bracket for the Whitham system. Besides that, we have

$$
\left\{k^{\alpha}(X), k^{\beta}(Y)\right\}_{\mathrm{DN}}=0
$$

according to relations (2.48).

The remaining part of the flat coordinates of $g^{\nu \mu}(\mathbf{U})$ and the corresponding annihilators of the bracket (2.21) are defined by concrete form of the initial bracket (2.16) and the family of $m$-phase solutions of system (2.3).

Let us prove now the second theorem justifying the invariance of the Dubrovin-Novikov procedure.

Theorem 3.2. Let $\Lambda$ be a regular Hamiltonian family of m-phase solutions of (2.3). Let $\left(I^{1}, \ldots, I^{N}\right)$ and $\left(I^{\prime 1}, \ldots, I^{\prime N}\right)$ be two different complete Hamiltonian sets of commuting first integrals of (2.3) having the form (2.18). Then the Dubrovin-Novikov brackets obtained using the sets $\left(I^{1}, \ldots, I^{N}\right)$ and $\left(I^{\prime 1}, \ldots, I^{\prime N}\right)$ coincide with each other.

Proof. Note that the sets $\left(I^{1}, \ldots, I^{N}\right),\left(I^{1}, \ldots, I^{N}\right)$ correspond to the two systems of coordinates $\left(U^{1}, \ldots, U^{N}\right),\left(U^{\prime 1}, \ldots, U^{\prime N}\right)$ on the family $\Lambda$, given by the averages of the functionals $\mathbf{I}$ and $\mathbf{I}^{\prime}$. We have to show then that the Dubrovin-Novikov brackets obtained using the set $\left(I^{\prime 1}, \ldots, I^{\prime N}\right)$ coincides with the bracket, obtained using the set $\left(I^{1}, \ldots, I^{N}\right)$, after the corresponding change of coordinates,

$$
U^{\prime \nu}=U^{\prime \nu}(\mathbf{U})
$$

Consider the values of the functionals

$$
J^{\nu}(X)=\int_{0}^{2 \pi} \cdots \int_{0}^{2 \pi} P^{\nu}\left(\boldsymbol{\varphi}, \epsilon \boldsymbol{\varphi}_{X}, \ldots\right) \frac{\mathrm{d}^{m} \theta}{(2 \pi)^{m}}
$$

and the values of constraints $g^{i}(\boldsymbol{\theta}, X)$, introduced with the aid of the functionals $\mathbf{J}(X)$ by formula (2.32), as a coordinate system in the neighborhood of the submanifold $\mathcal{K}$.

For values of the functionals $J^{\prime \nu}(X)$ on the submanifold $\mathcal{K}$ we then have the relations

$$
\left.J^{\prime \nu}(X)\right|_{\mathcal{K}}=U^{\prime \nu}(\mathbf{J}(X))+\sum_{l \geq 1} \epsilon^{l} j_{(l)}^{\prime \nu}\left(\mathbf{J}, \mathbf{J}_{X}, \ldots\right)
$$

where $j_{(l)}^{\prime \nu}$ are smooth functions of $\left(\mathbf{J}, \mathbf{J}_{X}, \ldots\right)$, polynomial in the derivatives and having degree $l$.

Expanding the values of $J^{\prime \nu}(X)$ in the neighborhood of the submanifold $\mathcal{K}$, we can write

$$
\begin{aligned}
J^{\prime \nu}(X)= & U^{\prime \nu}(\mathbf{J}(X))+\sum_{l \geq 1} \epsilon^{l} j_{(l)}^{\prime \nu}\left(\mathbf{J}, \mathbf{J}_{X}, \ldots\right) \\
& +\int_{-\infty}^{+\infty} \int_{0}^{2 \pi} \cdots \int_{0}^{2 \pi} T_{i}^{\prime \nu}(X, \boldsymbol{\theta}, Y, \epsilon) g^{i}(\boldsymbol{\theta}, Y) \frac{\mathrm{d}^{m} \theta}{(2 \pi)^{m}} \mathrm{~d} Y+O\left(\mathbf{g}^{2}\right),
\end{aligned}
$$

where, according to the form of the constraints (2.32), we can put

$$
\begin{aligned}
T_{i}^{\prime \nu}(X, \boldsymbol{\theta}, Y, \epsilon) & =\left.\sum_{l \geq 0} \Pi_{i(l)}^{\prime \nu}\left(\boldsymbol{\varphi}(\boldsymbol{\theta}, X), \epsilon \boldsymbol{\varphi}_{X}(\boldsymbol{\theta}, X), \ldots\right)\right|_{\mathcal{K}} \epsilon^{l} \delta^{(l)}(X-Y) \\
& =\left.\sum_{l \geq 0} \frac{\partial P^{\prime \nu}}{\partial \varphi_{l x}^{i}}\left(\boldsymbol{\varphi}(\boldsymbol{\theta}, X), \epsilon \boldsymbol{\varphi}_{X}(\boldsymbol{\theta}, X), \ldots\right)\right|_{\mathcal{K}} \epsilon^{l} \delta^{(l)}(X-Y) .
\end{aligned}
$$


Considering the functionals $J_{[\mathbf{q}]}^{\prime}=\int q_{\nu}(X) J^{\prime \nu}(X) \mathrm{d} X$ with arbitrary smooth (compactly supported) functions $q_{\nu}(X)$, we can write in the vicinity of $\mathcal{K}$

$$
\begin{aligned}
& J_{[\mathbf{q}]}^{\prime}=\int q_{\nu}(X)\left[U^{\prime \nu}(\mathbf{J}(X))+\sum_{l \geq 1} \epsilon^{l} j_{(l)}^{\prime \nu}\left(\mathbf{J}, \mathbf{J}_{X}, \ldots\right)\right] \mathrm{d} X \\
& +\int \sum_{l \geq 0}(-1)^{l} \epsilon^{l}\left[\left.\frac{\mathrm{d}^{l}}{\mathrm{~d} Y^{l}} q_{\nu}(Y) \Pi_{i(l)}^{\prime \nu}\left(\boldsymbol{\varphi}(\boldsymbol{\theta}, Y), \epsilon \boldsymbol{\varphi}_{Y}(\boldsymbol{\theta}, Y), \ldots\right)\right|_{\mathcal{K}}\right] g^{i}(\boldsymbol{\theta}, Y) \frac{\mathrm{d}^{m} \theta}{(2 \pi)^{m}} \mathrm{~d} Y+O\left(\mathbf{g}^{2}\right) .
\end{aligned}
$$

The leading term (in $\epsilon$ ) in the second part of the expression (3.28) is given by the expression

$$
\int q_{\nu}(Y) \sum_{l \geq 0}(-1)^{l} k^{\alpha_{1}}(Y) \cdots k^{\alpha_{l}}(Y) \Pi_{i(l) \theta^{\alpha_{1} \ldots \theta^{\alpha_{l}}}}^{\prime \nu}\left(\boldsymbol{\Phi}\left(\frac{\mathbf{S}(Y)}{\epsilon}+\boldsymbol{\theta}, Y\right), \ldots\right) g^{i}(\boldsymbol{\theta}, Y) \frac{\mathrm{d}^{m} \theta}{(2 \pi)^{m}} \mathrm{~d} Y
$$

and coincides with the value

$$
\iint_{0}^{2 \pi} \cdots \int_{0}^{2 \pi} q_{\nu}(Y) \zeta_{i[\mathbf{U}(Y)]}^{\prime(\nu)}\left(\frac{\mathbf{S}(Y)}{\epsilon}+\boldsymbol{\theta}\right) g^{i}(\boldsymbol{\theta}, Y) \frac{\mathrm{d}^{m} \theta}{(2 \pi)^{m}} \mathrm{~d} Y
$$

where

$$
\zeta_{i[\mathbf{U}]}^{\prime(\nu)}(\boldsymbol{\theta})=\left.\left[\frac{\delta}{\delta \varphi^{i}(\boldsymbol{\theta})} \int_{0}^{2 \pi} \cdots \int_{0}^{2 \pi} P^{\prime \nu}\left(\boldsymbol{\varphi}, k^{\beta} \boldsymbol{\varphi}_{\theta^{\beta}}, \ldots\right) \frac{\mathrm{d}^{m} \theta}{(2 \pi)^{m}}\right]\right|_{\boldsymbol{\varphi}(\boldsymbol{\theta})=\boldsymbol{\Phi}(\boldsymbol{\theta}, \mathbf{U})} .
$$

As the values $\zeta_{i[\mathbf{U}]}^{(\nu)}(\boldsymbol{\theta})$, the values $\zeta_{i[\mathbf{U}]}^{(\nu)}(\boldsymbol{\theta})$ represent regular left eigenvectors of the operator $\hat{L}_{j[\mathbf{U}]}^{i}$ corresponding to the zero eigenvalues. In the case of a complete regular family of $m$-phase solutions, we have therefore

$$
\zeta_{i[\mathbf{U}]}^{\prime(\nu)}(\boldsymbol{\theta})=\sum_{q} \Gamma_{q}^{\nu}(\mathbf{U}) \kappa_{i[\mathbf{U}]}^{(q)}(\boldsymbol{\theta})
$$

for some functions $\Gamma_{q}^{\nu}(\mathbf{U})$.

We can write, therefore, up to quadratic terms in $\mathbf{g}(\boldsymbol{\theta}, X)$

$$
\begin{aligned}
& \int q_{\nu}(X) J^{\prime \nu}(X) \mathrm{d} X=\int q_{\nu}(X)\left[U^{\prime \nu}(\mathbf{J}(X))+\sum_{l \geq 1} \epsilon^{l} j_{(l)}^{\prime \nu}\left(\mathbf{J}, \mathbf{J}_{X}, \ldots\right)\right] \mathrm{d} X \\
& \quad+\int q_{\nu}(X)\left[\sum_{q} \Gamma_{q}^{\nu}(\mathbf{U}) \kappa_{i[\mathbf{U}(X)]}^{(q)}\left(\frac{\mathbf{S}(X)}{\epsilon}+\boldsymbol{\theta}\right)+O(\epsilon)\right] g^{i}(\boldsymbol{\theta}, X) \frac{\mathrm{d}^{m} \theta}{(2 \pi)^{m}} \mathrm{~d} X+O\left(\mathbf{g}^{2}\right) .
\end{aligned}
$$

Consider the Poisson brackets

$$
\begin{aligned}
& \left.\left\{J_{[\mathbf{q}]}^{\prime}, J_{[\mathbf{p}]}^{\prime}\right\}\right|_{\mathcal{K}}=\left.\left\{\int q_{\nu}(X) J^{\prime \nu}(X) \mathrm{d} X, \int p_{\mu}(Y) J^{\prime \mu}(Y) \mathrm{d} Y\right\}\right|_{\mathcal{K}} \\
& \quad=\int q_{\nu}(X) \frac{\partial U^{\prime \nu}}{\partial U^{\lambda}}(X)\left[\left.\left\{J^{\lambda}(X), \int p_{\mu}(Y) J^{\prime \mu}(Y) \mathrm{d} Y\right\}\right|_{\mathcal{K}}+O\left(\epsilon^{2}\right)\right] \mathrm{d} X \\
& +\int q_{\nu}(X) \sum_{q} \Gamma_{q}^{\nu}(\mathbf{U}(X))\left[\kappa_{i[\mathbf{U}(X)]}^{(q)}\left(\frac{\mathbf{S}(X)}{\epsilon}+\boldsymbol{\theta}\right)+O(\epsilon)\right] \\
& \quad \times\left.\left\{g^{i}(\boldsymbol{\theta}, X), J^{\prime \mu}(Y)\right\}\right|_{\mathcal{K}} p_{\mu}(Y) \frac{\mathrm{d}^{m} \theta}{(2 \pi)^{m}} \mathrm{~d} X \mathrm{~d} Y .
\end{aligned}
$$


By Lemma 2.4 $4^{\prime}$ we have the relation $\left.\left\{g^{i}(\boldsymbol{\theta}, X), J_{[\mathbf{p}]}^{\prime}\right\}\right|_{\mathcal{K}}=O(\epsilon)$ on the submanifold $\mathcal{K}$. In addition, completely analogous to the relation (2.71) holds the relation

$$
\left.\int_{0}^{2 \pi} \cdots \int_{0}^{2 \pi} \kappa_{i[\mathbf{U}(X)]}^{(q)}\left(\frac{\mathbf{S}(X)}{\epsilon}+\boldsymbol{\theta}\right)\left\{g^{i}(\boldsymbol{\theta}, X), J_{[\mathbf{p}]}^{\prime}\right\}\right|_{\mathcal{K}[1]} \frac{\mathrm{d}^{m} \theta}{(2 \pi)^{m}} \equiv 0
$$

by virtue of the original dependence of the constraints $g^{i}(\boldsymbol{\theta}, X)$. We thus obtain

$$
\begin{aligned}
& \left.\iint q_{\nu}(X)\left\{J^{\prime \nu}(X), J^{\prime \mu}(Y)\right\}\right|_{\mathcal{K}} p_{\mu}(Y) \mathrm{d} X \mathrm{~d} Y \\
& \quad=\left.\int q_{\nu}(X) \frac{\partial U^{\prime \nu}}{\partial U^{\lambda}}(X)\left\{J^{\lambda}(X), \int p_{\mu}(Y) J^{\prime \mu}(Y) \mathrm{d} Y\right\}\right|_{\mathcal{K}} \mathrm{d} X+O\left(\epsilon^{2}\right) .
\end{aligned}
$$

Repeating the arguments for the functional $\int p_{\mu}(Y) J^{\prime \mu}(Y) \mathrm{d} Y$ we finally obtain

$$
\begin{aligned}
& \left.\iint q_{\nu}(X)\left\{J^{\prime \nu}(X), J^{\prime \mu}(Y)\right\}\right|_{\mathcal{K}} p_{\mu}(Y) \mathrm{d} X \mathrm{~d} Y \\
& \quad=\left.\iint q_{\nu}(X) \frac{\partial U^{\prime \nu}}{\partial U^{\lambda}}(X)\left\{J^{\lambda}(X), J^{\gamma}(Y)\right\}\right|_{\mathcal{K}} \frac{\partial U^{\prime \mu}}{\partial U^{\gamma}}(Y) p_{\mu}(Y) \mathrm{d} X \mathrm{~d} Y+O\left(\epsilon^{2}\right) .
\end{aligned}
$$

Given that the principal (in $\epsilon$ ) terms in the expressions $\left.\left\{J^{\lambda}(X), J^{\gamma}(Y)\right\}\right|_{\mathcal{K}}$ and $\left.\left\{J^{\prime \nu}(X), J^{\prime \mu}(Y)\right\}\right|_{\mathcal{K}}$ coincide with the Dubrovin-Novikov brackets, obtained with the aid of the sets $\left(I^{1}, \ldots, I^{N}\right)$ and $\left(I^{\prime 1}, \ldots, I^{\prime N}\right)$ respectively, we conclude from (3.29) that

$$
\left\{U^{\prime \nu}(X), U^{\prime \mu}(Y)\right\}_{\mathrm{DN}}^{\prime}=\frac{\partial U^{\prime \nu}}{\partial U^{\lambda}}(X)\left\{U^{\lambda}(X), U^{\gamma}(Y)\right\}_{\mathrm{DN}} \frac{\partial U^{\prime \mu}}{\partial U^{\gamma}}(Y),
$$

which means the coinciding of the brackets $\{\cdot, \cdot\}_{\mathrm{DN}}$ and $\{\cdot, \cdot\}_{\mathrm{DN}}^{\prime} \cdot$

Finally, we prove the theorem about the Hamiltonian properties of the Whitham system (2.10) under the same conditions as before.

Theorem 3.3. Let $\Lambda$ be a regular Hamiltonian family of $m$-phase solutions of (2.3). Let $\left(I^{1}, \ldots, I^{N}\right)$ be a complete Hamiltonian set of commuting first integrals of (2.3) having the form (2.18) and $H$ be the Hamiltonian function of the system (2.3) having the form (2.17). Then the Whitham system (2.10) is Hamiltonian with respect to the corresponding DubrovinNovikov bracket (2.21) with the Hamiltonian function (2.22)

$$
H^{a v}=\int_{-\infty}^{+\infty}\left\langle P_{H}\right\rangle(\mathbf{U}(X)) \mathrm{d} X
$$

Proof. By Theorem 3.2, without loss of generality we can assume that the Hamiltonian functional $H$ belongs to the set $\left(I^{1}, \ldots, I^{N}\right), H=I^{\mu_{0}}$. It is easy to verify that the corresponding Hamiltonian $H^{a v}$ generates in this case the system

$$
U_{T}^{\nu}=\frac{d}{\mathrm{~d} X}\left[\left\langle Q^{\nu \mu_{0}}\right\rangle(\mathbf{U}(X))\right]
$$

i.e. exactly system $(2.10)$.

As the simplest example of Theorems 3.1-3.3 consider the procedure of averaging of the Gardner-Zakharov-Faddeev bracket for the KdV equation.

Consider the Gardner-Zakharov-Faddeev bracket

$$
\{\varphi(x), \varphi(y)\}=\delta^{\prime}(x-y)
$$


for the $\mathrm{KdV}$ equation

$$
\varphi_{t}=\varphi \varphi_{x}-\varphi_{x x x}
$$

with the Hamiltonian functional

$$
H=\int\left(\frac{\varphi^{3}}{6}+\frac{\varphi_{x}^{2}}{2}\right) \mathrm{d} x
$$

As is well known, the $\mathrm{KdV}$ equation has a family of $m$-phase solutions for any $m$, given by the Novikov potentials, which represent the stationary points for the higher KdV flows. According to the Hamiltonian structure (3.30) it is also equivalent to the extremality of a linear combination of higher integrals of the $\mathrm{KdV}$

$$
c_{1} \delta I^{1}+c_{2} \delta I^{2}+\cdots+c_{m+2} \delta I^{m+2}=0,
$$

where

$$
I^{1}=N=\int \varphi \mathrm{d} x
$$

is the annihilator of the bracket (3.30),

$$
I^{2}=P=\int \frac{\varphi^{2}}{2} \mathrm{~d} x
$$

is the momentum functional of the bracket (3.30), $I^{3}=H$, and $I^{k}, k \geq 4$ are higher integrals of the KdV equation.

As was shown in [41], all the systems (3.31) are completely integrable finite-dimensional systems with quasiperiodic solutions in the general case. As is well known, the corresponding $m$-phase solutions of $\mathrm{KdV}$ can be represented in the form (1.2) with the theta-functional expressions for the functions $\Phi(\boldsymbol{\theta}, \mathbf{U})$, satisfying the system $[10-13,17,26,27]$

$$
\omega^{\alpha}(\mathbf{U}) \Phi_{\theta^{\alpha}}=k^{\alpha}(\mathbf{U}) \Phi \Phi_{\theta^{\alpha}}-k^{\alpha}(\mathbf{U}) k^{\beta}(\mathbf{U}) k^{\gamma}(\mathbf{U}) \Phi_{\theta^{\alpha} \theta^{\beta} \theta^{\gamma}} .
$$

The parameters of the solution $\left(E_{1}, \ldots, E_{2 m+1}\right), E_{1}<E_{2}<\cdots<E_{2 m+1}$ represent the branching points of the Riemann surface of genus $m$, and, together with the initial phases $\left(\theta_{0}^{1}, \ldots, \theta_{0}^{m}\right)$, completely determine the corresponding solution $\varphi(x, t)[41]$.

The eigenmodes of the linearized operator (3.32), as well as the adjoint operator, were studied in detail $[4,5,28-30]$. In particular, we can state that the families of $m$-phase solutions of $\mathrm{KdV}$ are complete regular families in the sense of Definition 1.1 and the corresponding systems (2.10) for any independent set of $2 m+1$ higher integrals of $\mathrm{KdV}\left(I^{k_{1}}, \ldots, I^{k_{2 m+1}}\right)$ represent the regular Whitham systems for the $\mathrm{KdV}$ equation in the sense defined above. It is well known [22,49], that the Whitham system for $\mathrm{KdV}$ in $m$-phase case can be written in the diagonal form where the parameters $E_{1}, \ldots, E_{2 m+1}$ are its Riemann invariants.

Regular zero modes of the linearized operator (3.32) on the torus $\mathbb{T}^{m}$ are given by the functions $\Phi_{\theta^{\alpha}}(\boldsymbol{\theta}, \mathbf{U}), \theta^{\alpha}=1, \ldots, m$ and by the function $\partial \Phi / \partial n$ (at constant $\mathbf{k}$ and $\boldsymbol{\omega}$ ), where $n$ is the value of the functional $N$ on the corresponding solution. Similarly, regular zero modes of the adjoint linear operator on the torus $\mathbb{T}^{m}$ are given by any $m+1$ linearly independent variational derivatives of the higher integrals of $\mathrm{KdV}$ on the family of the functions $\Phi(\boldsymbol{\theta}, \mathbf{U})$.

The families of Novikov potentials are obviously regular Hamiltonian families with respect to the bracket (3.30), while any set of $2 m+1$ independent higher integrals of $\mathrm{KdV}$ is a complete set of commuting functionals (2.18) on such a family. We also note that the variational derivative of the annihilator of the bracket (3.30) always appears on the family of $m$-phase solutions in the 
form of a linear combination of $m+1$ variational derivatives of higher integrals of $\mathrm{KdV}$ according to the original construction of the Novikov potentials.

Investigation of the system (3.1) can be carried out in this case as follows. We first note that the operator $\hat{B}_{[0]}(X)$ is given in this case as

$$
\hat{B}_{[0]}(X)=k^{1}(X) \frac{\partial}{\partial \theta^{1}}+\cdots+k^{m}(X) \frac{\partial}{\partial \theta^{m}}
$$

and, thus, is the derivative along the vector field $\left(k^{1}(X), \ldots, k^{m}(X)\right)$ on the torus $\mathbb{T}^{m}$.

The regular zero mode of the operator $\hat{B}_{[0]}(X)$ is a constant function on the torus and is orthogonal to the functions $A_{[1][\mathbf{q}]}(\boldsymbol{\theta}, X)$ by Lemma 3.1. This fact is easily verified also by direct computation. Indeed, the functions $A_{[1][\mathbf{q}]}(\boldsymbol{\theta}, X)$ have in this case the form

$$
A_{[1][\mathbf{q}]}\left(\frac{\mathbf{S}(X)}{\epsilon}+\boldsymbol{\theta}, X\right)=\left.\left\{\varphi(\boldsymbol{\theta}, X), J_{[\mathbf{q}]}\right\}\right|_{\mathcal{K}[1]}-\left.\Phi_{U^{\nu}}\left(\frac{\mathbf{S}(X)}{\epsilon}+\boldsymbol{\theta}, \mathbf{U}(X)\right)\left\{U^{\nu}(X), J_{[\mathbf{q}]}\right\}\right|_{\mathcal{K}[1]} .
$$

Let us assume for simplicity that the functional

$$
N(X)=\int_{0}^{2 \pi} \cdots \int_{0}^{2 \pi} \varphi(\boldsymbol{\theta}, X) \frac{\mathrm{d}^{m} \theta}{(2 \pi)^{m}}
$$

is included in the coordinate system $\mathbf{U}(X), N(X)=U^{1}(X)$, which, by Theorem 3.2, does not affect the Dubrovin-Novikov bracket. We have then

$$
\int_{0}^{2 \pi} \cdots \int_{0}^{2 \pi} \Phi_{U^{1}}(\boldsymbol{\theta}, \mathbf{U}(X)) \frac{\mathrm{d}^{m} \theta}{(2 \pi)^{m}} \equiv 1, \quad \int_{0}^{2 \pi} \cdots \int_{0}^{2 \pi} \Phi_{U^{\nu}}(\boldsymbol{\theta}, \mathbf{U}(X)) \frac{\mathrm{d}^{m} \theta}{(2 \pi)^{m}} \equiv 0, \quad \nu \neq 1 .
$$

It is easy to see then that

$$
\int_{0}^{2 \pi} \cdots \int_{0}^{2 \pi} A_{[1][\mathbf{q}]}(\boldsymbol{\theta}, X) \frac{\mathrm{d}^{m} \theta}{(2 \pi)^{m}}=\left.\left\{N(X), J_{[\mathbf{q}]}\right\}\right|_{\mathcal{K}[1]}-\left.\left\{N(X), J_{[\mathbf{q}]}\right\}\right|_{\mathcal{K}[1]} \equiv 0 .
$$

The operator $\hat{B}_{[0]}(X)$, however, has also irregular zero modes which arise in the cases when the closure of trajectories of the vector field $\left(k^{1}(X), \ldots, k^{m}(X)\right)$ on $\mathbb{T}^{m}$ represent the lowerdimensional tori $\mathbb{T}^{k} \subset \mathbb{T}^{m}$. Modes of this type arise obviously on a set of measure zero in the parameter space $\mathbf{U}$, i.e. $\{\mathbf{U}\} / \mathcal{M}$.

We assume here, without proof, following easily verifiable from the theta-functional representation fact. The Fourier harmonics $A_{[1][\mathbf{q}]}^{n_{1} \ldots n_{m}}(X)$ of the functions $A_{[1][\mathbf{q}]}(\boldsymbol{\theta}, X)$ in the variables $\boldsymbol{\theta}$

$$
A_{[1][\mathbf{q}]}(\boldsymbol{\theta}, X) \equiv \sum_{n_{1}, \ldots, n_{m}} A_{[1][\mathbf{q}]}^{n_{1} \ldots n_{m}}(X) \exp \left(i n_{1} \theta^{1}+\cdots+i n_{m} \theta^{m}\right)
$$

decay faster than any power of $|\mathbf{n}|$ at $|\mathbf{n}| \rightarrow \infty$, where

$$
|\mathbf{n}| \equiv \sqrt{n_{1}^{2}+\cdots+n_{m}^{2}} .
$$

Let us define the Diophantine conditions for an arbitrary set of values $\left(k^{1}(\mathbf{U}), \ldots, k^{m}(\mathbf{U})\right)$. The vector $\left(k^{1}(\mathbf{U}), \ldots, k^{m}(\mathbf{U})\right)$ is a Diophantine vector with the index $\nu>0$ and the coefficient $A>0$, if

$$
\left|n_{1} k^{1}(\mathbf{U})+\cdots+n_{m} k^{m}(\mathbf{U})\right| \geq A|\mathbf{n}|^{-\nu}
$$

for all $\left(n_{1}, \ldots, n_{m}\right) \in \mathbb{Z}^{m}\left(\left(n_{1}, \ldots, n_{m}\right) \neq(0, \ldots, 0)\right)$.

Let us use now the following well known theorem (see, e.g., $[3,45])$ : 
For $\nu>m-1$ the measure of the set of non-Diophantine vectors $\left(k^{1}, \ldots, k^{m}\right)$ in $\mathbb{R}^{m}$ is equal to zero.

Using the condition rank $\left\|\partial k^{\alpha} / \partial U^{\nu}\right\|=m$ we can also state that the measure of the corresponding parameters $\mathbf{U}$, such that $\mathbf{k}(\mathbf{U})$ are non-Diophantine vectors with index $\nu>m-1$, is also zero in the parameter space $\mathbf{U}$.

We can now define the sets $\mathcal{S}_{\nu}$ in the space of the parameters $\mathbf{U}$, such that the wave vectors $\left(k^{1}(\mathbf{U}), \ldots, k^{m}(\mathbf{U})\right)$ are Diophantine with index $\nu$ if $\mathbf{U} \in \mathcal{S}_{\nu}$. In this case all $\mathcal{S}_{\nu}$ with index $\nu>m-1$ are everywhere dense, and also have the full measure in the parameter space $\mathbf{U}$.

It is easy to see also that the system (3.1) is resolvable in the space of $2 \pi$-periodic in all $\theta^{\alpha}$ functions for each $\mathbf{U} \in \mathcal{S}_{\nu}$. Indeed, due to the absence of the zero Fourier harmonic of the function $A_{[1][\mathbf{q}]}(\boldsymbol{\theta}, X)$ and conditions of decreasing of Fourier coefficients $A_{[1][\mathbf{q}]}^{n_{1} \ldots n_{m}}(X)$ we can put

$$
B_{[\mathbf{q}](1)}^{n_{1} \ldots n_{m}}(X)=\frac{1}{i n_{1} k^{1}(\mathbf{U})+\cdots+i n_{m} k^{m}(\mathbf{U})} A_{[1][\mathbf{q}]}^{n_{1} \ldots n_{m}}(X)
$$

for $\left(n_{1}, \ldots, n_{m}\right) \neq(0, \ldots, 0)$ and restore the solution

$$
\begin{aligned}
B_{[\mathbf{q}](1)}(\boldsymbol{\theta}, X) & =\sum_{n_{1}, \ldots, n_{m}}^{\prime} B_{[\mathbf{q}](1)}^{n_{1} \ldots n_{m}}(X) \exp \left(i n_{1} \theta^{1}+\cdots+i n_{m} \theta^{m}\right), \\
\left(n_{1}, \ldots, n_{m}\right) & \neq(0, \ldots, 0)
\end{aligned}
$$

as a smooth $m$-periodic function of $\boldsymbol{\theta}$.

Assuming, therefore, $\mathcal{S}=\mathcal{S}_{\nu}$ for any $\nu>m-1$, we meet all the conditions of Theorem 3.1.

We can thus formulate the following proposition.

Proposition 3.1. The Dubrovin-Novikov procedure is well justified in the averaging of the Gardner-Zakharov-Faddeev bracket on the $m$-phase solutions of $K d V$ for any $m$ and provides a local Hamiltonian structure of hydrodynamic type for the corresponding regular Whitham system.

We note here that both local and weakly nonlocal Hamiltonian structures of the Whitham hierarchy for $\mathrm{KdV}$ were investigated in detail in the papers $[2,44]$.

In the more general case the constructing of the set $\mathcal{S}$ requires the study of the eigenfunctions

and the eigenvalues of the Hamilton operator $\hat{B}_{[0]}^{i j}(\mathbf{U})$ on the manifold of $m$-phase solutions depending on the values of the parameters $\mathbf{U}$. For $m$-phase solutions, given by the algebraicgeometric families, in this case are very convenient methods similar to those used in $[4,5,28,30,47]$ to study the spectrum of auxiliary linear operators of integrable hierarchies.

\section{Acknowledgements}

In conclusion the author expresses his deep gratitude to Professors S.P. Novikov, B.A. Dubrovin, I.M. Krichever, S.Yu. Dobrokhotov, and M.V. Pavlov for fruitful discussions. This work was financially supported by the Russian Federation Government Grant No. 2010-220-01-077, Grant of the President of Russian Federation NSh-4995.2012.1, and Grant RFBR No. 11-01-12067-ofi$\mathrm{m}-2011$.

\section{References}

[1] Ablowitz M.J., Benney D.J., The evolution of multi-phase modes for nonlinear dispersive waves, Stud. Appl. Math. 49 (1970), 225-238.

[2] Alekseev V.L., On non-local Hamiltonian operators of hydrodynamic type connected with Whitham's equations, Russian Math. Surveys 50 (1995), 1253-1255. 
[3] Arnol'd V.I., Geometrical methods in the theory of ordinary differential equations, Grundlehren der Mathematischen Wissenschaften, Vol. 250, 2nd ed., Springer-Verlag, New York, 1988.

[4] Dobrokhotov S.Yu., Resonance correction of an adiabatically perturbed finite-gap almost periodic solution of the Korteweg-de Vries equation, Math. Notes 44 (1988), 551-555.

[5] Dobrokhotov S.Yu., Resonances in asymptotic solutions of the Cauchy problem for the Schrödinger equation with rapidly oscillating finite-zone potential, Math. Notes 44 (1988), 656-668.

[6] Dobrokhotov S.Yu., Krichever I.M., Multi-phase solutions of the Benjamin-Ono equation and their averaging, Math. Notes 49 (1991), 583-594.

[7] Dobrokhotov S.Yu., Maslov V.P., Finite-gap almost periodic solutions in the WKB approximation, J. Sov. Math. 15 (1980), 1433-1487.

[8] Dobrokhotov S.Yu., Maslov V.P., Multiphase asymptotics of nonlinear partial differential equations with a small parameter, in Mathematical Physics Reviews, Soviet Sci. Rev. Sect. C Math. Phys. Rev., Vol. 3, Harwood Academic Publ., Chur, 1982, 221-311.

[9] Dobrokhotov S.Yu., Minenkov D.S., Remark on the phase shift in the Kuzmak-Whitham ansatz, Theoret. Math. Phys. 166 (2011), 303-316.

[10] Dubrovin B.A., Inverse problem for periodic finite-zoned potentials in the theory of scattering, Funct. Anal. Appl. 9 (1975), 61-62.

[11] Dubrovin B.A., Theta functions and non-linear equations, Russian Math. Surveys 36 (1981), no. 2, 11-92.

[12] Dubrovin B.A., Matveev V.B., Novikov S.P., Nonlinear equations of Korteweg-de Vries type, finite-band linear operators and Abelian varieties, Russian Math. Surveys 31 (1976), no. 1, 59-146.

[13] Dubrovin B.A., Novikov S.P., A periodic problem for the Korteweg-de Vries and Sturm-Liouville equations. Their connection with algebraic geometry, Soviet Math. Dokl. 15 (1976), 1597-1601.

[14] Dubrovin B.A., Novikov S.P., Hamiltonian formalism of one-dimensional systems of the hydrodynamic type and the Bogolyubov-Whitham averaging method, Soviet Math. Dokl. 27 (1983), 665-669.

[15] Dubrovin B.A., Novikov S.P., Hydrodynamics of soliton lattices, Sov. Sci. Rev. Sect. C 9 (1992), no. 4, $1-136$.

[16] Dubrovin B.A., Novikov S.P., Hydrodynamics of weakly deformed soliton lattices. Differential geometry and Hamiltonian theory, Russian Math. Surveys 44 (1989), no. 6, 35-124.

[17] Dubrovin B.A., Novikov S.P., Periodic and conditionally periodic analogs of the many-soliton solutions of the Korteweg-de Vries equation, Soviet Physics JETP 67 (1974), 1058-1063.

[18] Ferapontov E.V., Differential geometry of nonlocal Hamiltonian operators of hydrodynamic type, Funct. Anal. Appl. 25 (1991), 195-204.

[19] Ferapontov E.V., Nonlocal Hamiltonian operators of hydrodynamic type: differential geometry and applications, in Topics in Topology and Mathematical Physics, Amer. Math. Soc. Transl. Ser. 2, Vol. 170, Amer. Math. Soc., Providence, RI, 1995, 33-58.

[20] Ferapontov E.V., Nonlocal matrix Hamiltonian operators, differential geometry and applications, Theoret. Math. Phys. 91 (1992), 642-649.

[21] Ferapontov E.V., Restriction, in the sense of Dirac, of the Hamiltonian operator $\delta^{i j}(d / d x)$ to a surface of the Euclidean space with a plane normal connection, Funct. Anal. Appl. 26 (1992), 298-300.

[22] Flaschka H., Forest M.G., McLaughlin D.W., Multiphase averaging and the inverse spectral solution of the Korteweg-de Vries equation, Comm. Pure Appl. Math. 33 (1980), 739-784.

[23] Haberman R., Standard form and a method of averaging for strongly nonlinear oscillatory dispersive traveling waves, SIAM J. Appl. Math. 51 (1991), 1638-1652.

[24] Haberman R., The modulated phase shift for weakly dissipated nonlinear oscillatory waves of the Kortewegde Vries type, Stud. Appl. Math. 78 (1988), 73-90.

[25] Hayes W.D., Group velocity and nonlinear dispersive wave propagation, Proc. R. Soc. Lond. Ser. A 332 (1973), 199-221.

[26] Its A.R., Matveev V.B., Hill's operator with finitely many gaps, Funct. Anal. Appl. 9 (1975), 65-66.

[27] Its A.R., Matveev V.B., Schrödinger operators with finite-gap spectrum and $N$-soliton solutions of the Korteweg-de Vries equation, Theoret. Math. Phys. 23 (1975), 343-355.

[28] Krichever I.M., Perturbation theory in periodic problems for two-dimensional integrable systems, Sov. Sci. Rev. Sect. C 9 (1992), no. 2, 1-103. 
[29] Krichever I.M., The averaging method for two-dimensional "integrable" equations, Funct. Anal. Appl. 22 (1988), 200-213.

[30] Krichever I.M., The "Hessian" of integrals of the Korteweg-de Vries equation and perturbations of finite-gap solutions, Sov. Math. Dokl. 270 (1983), 757-761.

[31] Luke J.C., A perturbation method for nonlinear dispersive wave problems, Proc. R. Soc. Lond. Ser. A 292 (1966), 403-412.

[32] Maltsev A.Ya., Conservation of Hamiltonian structures in Whitham's averaging method, Izv. Math. 63 (1999), 1171-1201.

[33] Maltsev A.Ya., Deformations of the Whitham systems in the almost linear case, in Geometry, Topology, and Mathematical Physics, Amer. Math. Soc. Transl. Ser. 2, Vol. 224, Amer. Math. Soc., Providence, RI, 2008, 193-212, arXiv:0709.4618.

[34] Maltsev A.Ya., The averaging of nonlocal Hamiltonian structures in Whitham's method, Int. J. Math. Math. Sci. 30 (2002), 399-434, solv-int/9910011.

[35] Maltsev A.Ya., Whitham systems and deformations, J. Math. Phys. 47 (2006), 073505, 18 pages, nlin.SI/0509033.

[36] Maltsev A.Ya., Novikov S.P., On the local systems Hamiltonian in the weakly non-local Poisson brackets, Phys. D 156 (2001), 53-80, nlin.SI/0006030.

[37] Maltsev A.Ya., Pavlov M.V., On Whitham's averaging method, Funct. Anal. Appl. 29 (1995), 6-19, nlin.SI/0306053.

[38] Mokhov O.I., Ferapontov E.V., Non-local Hamiltonian operators of hydrodynamic type related to metrics of constant curvature, Russian Math. Surveys 45 (1990), 218-219.

[39] Newell A.C., Solitons in mathematics and physics, CBMS-NSF Regional Conference Series in Applied Mathematics, Vol. 48, Society for Industrial and Applied Mathematics (SIAM), Philadelphia, PA, 1985.

[40] Novikov S.P., Geometry of conservative systems of hydrodynamic type. The averaging method for fieldtheoretic systems, Russian Math. Surveys 40 (1985), no. 4, 85-98.

[41] Novikov S.P., The periodic problem for the Korteweg-de Vries equation, Funct. Anal. Appl. 8 (1974), 236-246.

[42] Novikov S.P., Manakov S.V., Pitaevskiŭ L.P., Zakharov V.E., Theory of solitons. The inverse scattering method, Contemporary Soviet Mathematics, Plenum, New York, 1984.

[43] Pavlov M.V., Elliptic coordinates and multi-Hamiltonian structures of hydrodynamic-type systems, Russian Acad. Sci. Dokl. Math. 50 (1995), 374-377.

[44] Pavlov M.V., Multi-Hamiltonian structures of the Whitham equations, Russian Acad. Sci. Dokl. Math. 50 (1995), 220-223.

[45] Schmidt W.M., Diophantine approximation, Lecture Notes in Mathematics, Vol. 785, Springer-Verlag, Berlin - Heidelberg - New York, 1980.

[46] Tsarev S.P., On Poisson bracket and one-dimensional systems of hydrodynamic type, Soviet Math. Dokl. 31 (1985), 488-491.

[47] Vorob'ev Y.M., Dobrokhotov S.Yu., Completeness of the system of eigenfunctions of a nonelliptic operator on the torus, generated by a Hill operator with a finite-zone potential, Funct. Anal. Appl. 22 (1988), 137-139.

[48] Whitham G.B., A general approach to linear and non-linear dispersive waves using a Lagrangian, J. Fluid Mech. 22 (1965), 273-283.

[49] Whitham G.B., Linear and nonlinear waves, Pure and Applied Mathematics, Wiley-Interscience, New York, 1974.

[50] Whitham G.B., Non-linear dispersive waves, Proc. R. Soc. Lond. Ser. A 283 (1965), 238-261. 